\title{
Masi Entropy for Satellite Color Image Segmentation Using Tournament-Based Lévy Multiverse Optimization Algorithm
}

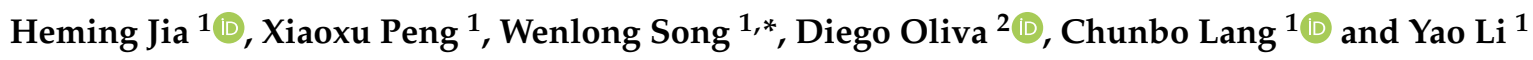 \\ 1 College of Mechanical and Electrical Engineering, Northeast Forestry University, Harbin 150040, China; \\ jiaheming@nefu.edu.cn (H.J.); pengxiaoxu@nefu.edu.cn (X.P.); langchunbo@nefu.edu.cn (C.L.); \\ liyao@nefu.edu.cn (Y.L.) \\ 2 Departamento de Ciencias Computacionales, Universidad de Guadalajara, CUCEI, Av. Revolución 1500, \\ 44430 Guadalajara, Jalisco, Mexico; diego.oliva@cucei.udg.mx \\ * Correspondence: swl@nefu.edu.cn
}

Received: 3 April 2019; Accepted: 16 April 2019; Published: 18 April 2019

\begin{abstract}
A novel multilevel threshold segmentation method for color satellite images based on Masi entropy is proposed in this paper. Lévy multiverse optimization algorithm (LMVO) has a strong advantage over the traditional multiverse optimization algorithm (MVO) in finding the optimal solution for the segmentation in the three channels of an RGB image. As the work advancement introduces a Lévy multiverse optimization algorithm which uses tournament selection instead of roulette wheel selection, and updates some formulas in the algorithm with mutation factor. Then, the proposal is called TLMVO, and another advantage is that the population diversity of the algorithm in the latest iterations is maintained. The Masi entropy is used as an application and combined with the improved TLMVO algorithm for satellite color image segmentation. Masi entropy combines the additivity of Renyi entropy and the non-extensibility of Tsallis entropy. By increasing the number of thesholds, the quality of segmenttion becomes better, then the dimensionality of the problem also increases. Fitness function value, average CPU running time, Peak Signal-to-Noise Ratio (PSNR), Structural Similarity Index (SSIM) and Feature Similarity Index (FSIM) were used to evaluate the segmentation results. Further statistical evaluation was given by Wilcoxon's rank sum test and Friedman test. The experimental results show that the TLMVO algorithm has wide adaptability to high-dimensional optimization problems, and has obvious advantages in objective function value, image quality detection, convergence performance and robustness.
\end{abstract}

Keywords: multilevel threshold segmentation; Masi entropy; multiverse optimization algorithm; Lévy multiverse optimization algorithm; tournament selection

\section{Introduction}

With the booming of artificial intelligence (IA) technology, in order to meet people's needs, the practicality of computer vision technology is highly emphasized. Image segmentation is one of the main problems of digital image processing technology and machine vision technology [1], which can be either gray image segmentation or color image segmentation. By comparison, grayscale images contain less information. Meanwhile, color images contain more color information such as hue and saturation [2,3]. On the other hand, images have a wide range of applications in the fields of geographic graphic information systems, astronomy and earth science research. It is necessary to locate objects and boundaries accurately in satellite images. Therefore, color satellite image segmentation is a critical and challenging topic [4-6]. 
The existing image segmentation methods are mainly divided into the following categories: threshold segmentation, region growth, region division and merging, watershed algorithm, edge detection, histogram method, cluster analysis and wavelet transform among others. Threshold segmentation is widely used and it can be divided into bi-level threshold and multilevel threshold $[7,8]$. Bi-level threshold is the simplest segmentation method, as long as one gray value can be determined to divide the image into two regions of interest (ROI) [9]. In actual image processing, color images contain more than two ROI, for that reason only multilevel threshold methods can be adopted. The pixels are divided into groups, and, within each group, the pixels have intensity values within a specific range. Sezgin et al. [10] divided the image thresholding techniques into six groups according to information. These groups include methods based on histogram, clustering, entropy, object attributes, spatial and local information. The segmentation techniques that employ histograms and statistical information (variance of entropy) are the most used due to its practicality. An example of these kinds of approaches is Otsu's algorithm, where each bar of the histogram represents a gray scale. In Otsu, the best threshold is obtained by computing the between class variance that exists among the two classes [11]. The higher the between-class variance is, the better the segmentation effect will be. As a basic and effective segmentation method, Otsu has been highly regarded and widely used for a long time. Today, people still have not stopped researching and utilizing it. In 2019, an accurate, scalable, polynomial time multistage threshold segmentation algorithm based on Otsu method has just been proposed [12]. Entropy-based methods, with their virtue of the charming basic mathematical concepts of entropy, has been infinitely improved and updated by researchers. In the image, the uniform region corresponds to the minimum entropy, while the non-uniform region defines the maximum entropy. Therefore, a better segmentation effect can be obtained by obtaining a larger Boltzmann-Gibbs entropy of the segmented image $[13,14]$. Therefore, entropy-based algorithms with different characteristics are well known. For instance, Fuzzy entropy [15], Renyi entropy [16], Shannon entropy [17], Tsallis entropy [18], and Kapur entropy [19]. Entropy-based thresholding has been widely used in multilevel image segmentation.

The main drawback of segmentation using entropy is to find the best configuration of thresholds. Each threshold increases the computational effort-for that reason, it is required the use of a search algorithm. Considering the above, segmentation is considered as an optimization problem in the literature, where entropy is used as an objective function. An interesting approach that employs optimization considers a hybridization of genetic algorithms and cross entropy methods (GACE) were proposed for solving continuous optimization [20]. An improved fuzzy entropy and Lévy flying firefly algorithm (FA) method is used for color image threshold segmentation [21]. By maximizing Shannon entropy or fuzzy entropy, the FA is utilized to image segmentation [22]. On the other hand, in 2005, Masi et al. proposed a newer and more coordinated Masi entropy which integrates the additivity of Renyi entropy and the non-extensibility of Tsallis entropy [23]. Fundamentally, Masi entropy is also a kind of innovation of Shannon entropy. By adjusting the entropy parameter $r$ of Masi entropy, its application scope can be expanded. Swapnil Shubham et al. applied the concept of Masi entropy to multilevel thresholding of color images, and confirmed its potential to achieve a wide range of objectives in efficient multilevel image segmentation [24,25].

The prosperity of optimization field drives the development of many fields. The cross-fusion of different areas through optimization algorithm can bring more immeasurable value to people. With the introduction of the No Free Lunch (NFL) theorem, people have realized the universality of optimization [26]. In 2002, E. G. Talbi published an article about a taxonomy of hybrid metaheuristics [27]. E. A. Baniani proposed a hybrid particle swarm optimization (PSO) algorithm and a genetic algorithm (GA) in 2013 for multilevel maximum entropy criterion threshold selection [28]. A hybrid whale algorithm and simulated annealing optimization algorithm have been applied to feature selection in 2017 [29]. At present, the combination of optimization algorithm and image segmentation can be said to be very mature, with a relatively complete system. With the introduction of more new and effective algorithms and the improvement of image segmentation methods, the prospects in this field are very 
promising. An approach called Multiverse Optimization (MVO) was first proposed by S. Mirjalili in 2015, which belongs to the physically inspired metaheuristic algorithms [30]. With the proposal, it has received successive improvements and utilization by scholars. In 2016, MVO was applied to the study of photovoltaic parameters, and five parameters of the single-diode model of photovoltaic cells were extracted [31]. In 2017, MVO mixed the PSO to solve the problem of global numerical optimization and reactive optimization scheduling [32]. In 2018, China's energy consumption was estimated using a self-adaptive MVO optimizer support vector machine with rolling cross-validation [33]. In 2019, the multi-objective MVO algorithm was utilized for grayscale image segmentation [34]. Compared with the traditional algorithm, the MVO algorithm has better performance, but there are still some flaws in slow convergence, low accuracy and ease of being trapped in the local optimal. Roulette wheel selection is used as a mechanism for determining the optimal universe in MVO. However, when the fitness function of the algorithm is so close at the later stage, the selection advantage of the optimal universe is greatly weakened, and it is easy to fall into local solutions. To solve these drawbacks, this paper proposed the use of the tournament selection. By calculating the reciprocal of fitness function, the optimal universe can be determined. This is because, in the minimization problem, tournament selection is better than roulette wheel selection, and can maintain a strong and continuous update even at the end of the iterative process, which has been proved in the related literature $[35,36]$.

Regarding the improvement of MVO, an enhanced version that merges the MVO with Lévy flight (LMVO) has recently been proposed [37]. When Lévy random walk is added to MVO appropriately, the algorithm not only improves the accuracy, but also enhances the robustness. As a promotion of the work, the mutation factor is added to the location update while replacing the screening mechanism, which ensures the diversity of the population in the later stage of the algorithm. These improvements are conducive to achieving a better balance between the exploration and exploitation of the algorithm, improving the accuracy of local optimization and the ability of global optimization. Therefore, this article presents an improved version of the LMVO called TLMVO including a tournament selection operator instead of the roulette wheel. As a real application, the TLMVO has been used for image thresholding using the Masi entropy as a fitness function. The proposal is tested using color satellite images that are more complicated than benchmark datasets. The performance of the improved algorithm is evaluated by considering the accuracy of the optimization, the quality of the segmentation and a statistical comparative analysis.

The remainder of this paper is organized as follows: Section 2 outlines the multi-threshold problem and Masi entropy. Section 3 gives an overview of MVO followed by its mathematical model. The proposed TLMVO-based multilevel thresholding method is presented in Section 4, where the basic instructions of three strategy are also illustrated. Simulation experiments and results analysis are described in Section 5. Finally, Section 6 concludes the work and suggests some directions for future studies.

\section{Problem Statement}

\subsection{Summary Description of Multilevel Thresholding}

Assuming that a color image with dimension $M \times N$ has $L$ gray values $[0,1, \ldots, L-1]$ for each of the color frame (red, green, and blue). $L$ is considered as 256 .

In each frame, let $n_{i}$ represent the number of pixels with gray value of $i$. Correspondingly, the distribution probability $p_{i}$ of the $i$-th gray value is indicated as:

$$
\begin{gathered}
p_{i}=\frac{n_{i}}{M \times N^{\prime}} \\
\sum_{0}^{L-1} p_{i}=1, p_{i} \geq 0 .
\end{gathered}
$$


Suppose there are $K$ thresholds. Then, $t_{1}, t_{2}, \cdots, t_{K}$ can divide the the gray levels of the given image into $K+1$ classes, for which $t$ represents threshold value. For multilevel thresholding, define different classes as:

$$
\begin{gathered}
{\left[0, t_{1}-1\right] \in M_{0}} \\
{\left[t_{1}, t_{2}-1\right] \in M_{1}} \\
\ldots \ldots \\
{\left[t_{K}, L-1\right] \in M_{K}}
\end{gathered}
$$

where $t_{1}<t_{2}<\ldots<t_{K}$. Then $t_{0}=0$ and $t_{K+1}=L$.

\subsection{Masi Entropy}

Most of the entropy testing methods for image segmentation need to obtain the maximum entropy. The effect of the segmentation between the object and the background depends on the value of the entropy. Experimental results have shown that fitness function value of Kapur's, Tsallis, Renyi's, and Masi's entropy are sorted as Kapur's $<$ Tsallis $<$ Renyi's $<$ Masi's [24].

For multilevel thresholding image segmentation of $K$ thresholds, the class probabilities are defined as:

$$
\omega_{0}=\sum_{i=0}^{t_{1}-1} p_{i}, \omega_{1}=\sum_{i=t_{1}}^{t_{2}-1} p_{i}, \omega_{2}=\sum_{i=t_{2}}^{t_{3}-1} p_{i}, \ldots, \omega_{K}=\sum_{i=t_{K}}^{L-1} p_{i} .
$$

Furthermore, the probability distribution defined above is normalized, and each new set of probability distribution is obtained in different classes, which can be expressed by mathematical formulas as:

$$
D M_{0}: \frac{p_{0}}{\omega_{0}}, \frac{p_{1}}{\omega_{0}}, \ldots, \frac{p_{t_{1}-1}}{\omega_{0}}, D M_{1}: \frac{p_{t_{1}}}{\omega_{1}}, \frac{p_{t_{1}+1}}{\omega_{1}}, \ldots, \frac{p_{t_{2}-1}}{\omega_{1}}, D M_{K}: \frac{p_{t_{K}}}{\omega_{K}}, \frac{p_{t_{K}+1}}{\omega_{K}}, \ldots, \frac{p_{L-1}}{\omega_{K}}
$$

The entropy value of the image can be represented as:

$$
H_{j}=\frac{1}{1-r} \log \left[1-(1-r) \sum_{i=t_{j}}^{t_{j+1}-1}\left(\frac{p_{i}}{\omega_{j}}\right) \log \left(\frac{p_{i}}{\omega_{j}}\right)\right],
$$

where $0 \leq j \leq K$. The entropy is represented by $H$, and $\mathrm{r}$ is the value of the entropic parameter which is set to 1.2. Then, the objective function can be mathematically described by:

$$
\psi\left(t_{1}, t_{2}, \cdots, t_{K}\right)=H_{1}+H_{2}+\ldots+H_{K}
$$

for which the definition of the optimal threshold of Masi is as follows:

$$
\left\{t_{1}^{*}, t_{2}^{*}, \cdots, t_{K}^{*}\right\}=\underset{0<t_{1}<t_{2} \cdots<t_{K}<L-1}{\arg \max }\left(\psi\left(t_{1}, t_{2}, \cdots, t_{K}\right)\right) .
$$

For color images, as described above, Masi entropy is calculated for each color channel of the image. Using algorithms, the objective function defined in Equation (7) is maximized by Equation (8), the threshold of each channel is calculated separately, and the segmented RGB images are formed by using these thresholds. Using the resulting optimal threshold, a final segmented image is formed.

\section{Multiverse Optimization Algorithm}

\section{The Basic Multiverse Optimization Algorithm}

Inspired by the Big Bang and Quantum Mechanics [38,39], each universe is regarded as a possible solution vector, treating an object in the universe as a variable in the corresponding solution vector. Each universe has a corresponding inflation rate, which is seen as fitness function value. Black holes 
are used to receive objects and exist in the universe with a low rate of inflation; white holes are used to send out objects and exist in the universe with a high rate of inflation; wormholes are tunnels between black holes and white holes; and the value of the expansion ratio is screened by a roulette mechanism to produce a white hole. According to the above rules, balanced exploration and development achieve optimal universe renewal. The following formulas correspond to the mathematical algorithmic models of the multiverse.

\section{Mathematical Model}

Considering the following definition of a universe:

$$
U=\left[\begin{array}{cccc}
x_{1}^{1} & x_{1}^{2} & \cdots & x_{1}^{d} \\
x_{2}^{1} & x_{2}^{2} & \cdots & x_{2}^{d} \\
\cdots & \cdots & \cdots & \cdots \\
x_{n}^{1} & x_{n}^{2} & \cdots & x_{n}^{d}
\end{array}\right]
$$

$d$ represents the number of parameters and $n$ refers to the number of solutions. Each element of $U$ is then defined as:

$$
x_{i}^{j}=\left\{\begin{array}{ll}
x_{k}^{j} & r_{1}<N I\left\{U_{i}\right. \\
x_{i}^{j} & r_{1} \geq N I\left\{U_{i}\right.
\end{array},\right.
$$

where $x_{i}^{j}$ represents the $j$-th parameter of $i$-th universe, $U_{i}$ represents the $i$-th universe, $N I\left(U_{i}\right)$ refers to the standard inflation rate of the $i$-th universe and $x_{k}^{j}$ indicates the $j$-th parameter of $k$-th universe selected by a roulette wheel selection mechanism.

The new positions of the elements in the optimal universe are obtained by Equation (11):

$$
x_{i+1}^{j}=\left\{\begin{array}{ccc}
x_{i}^{j}+T D R \times\left\{\left\{u b_{j}-l b_{j} \times r_{4}+l b_{j}\right.\right. & r_{3}<H & \\
x_{i}^{j}-T D R \times\left\{\left\{u b_{j}-l b_{j} \times r_{4}+l b_{j}\right.\right. & r_{3} \geq H & r_{2}<W E P \\
x_{i}^{j} & r_{2} \geq W E P
\end{array},\right.
$$

where $H=0.5, r_{1}, r_{2}, r_{3}, r_{4}$ are random numbers in the interval $[0,1]$. Wormhole existence probability (WEP) is as follows:

$$
W E P=\min +l \times\left(\frac{\max -\min }{L}\right)
$$

Here, $\min =0.2, \max =1, l$ is the current iteration, and $L$ is the maximum iteration. Travelling distance rate (TDR) is:

$$
T D R=1-\frac{l^{1 / p}}{L^{1 / p}} .
$$

$p$ denotes the accuracy of mining capability. Both TDR and WEP are coefficients and the relationship between them is shown in Figure 1. Local and global optimization are realized through Equations (10) and (11). The pseudo-code of the MVO algorithm is given in Algorithm 1.

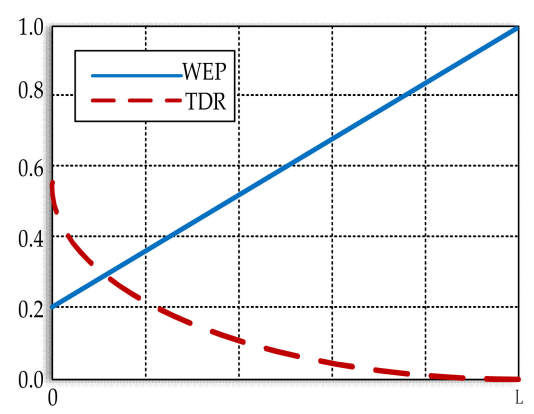

Figure 1. WEP versus TDP [30]. 


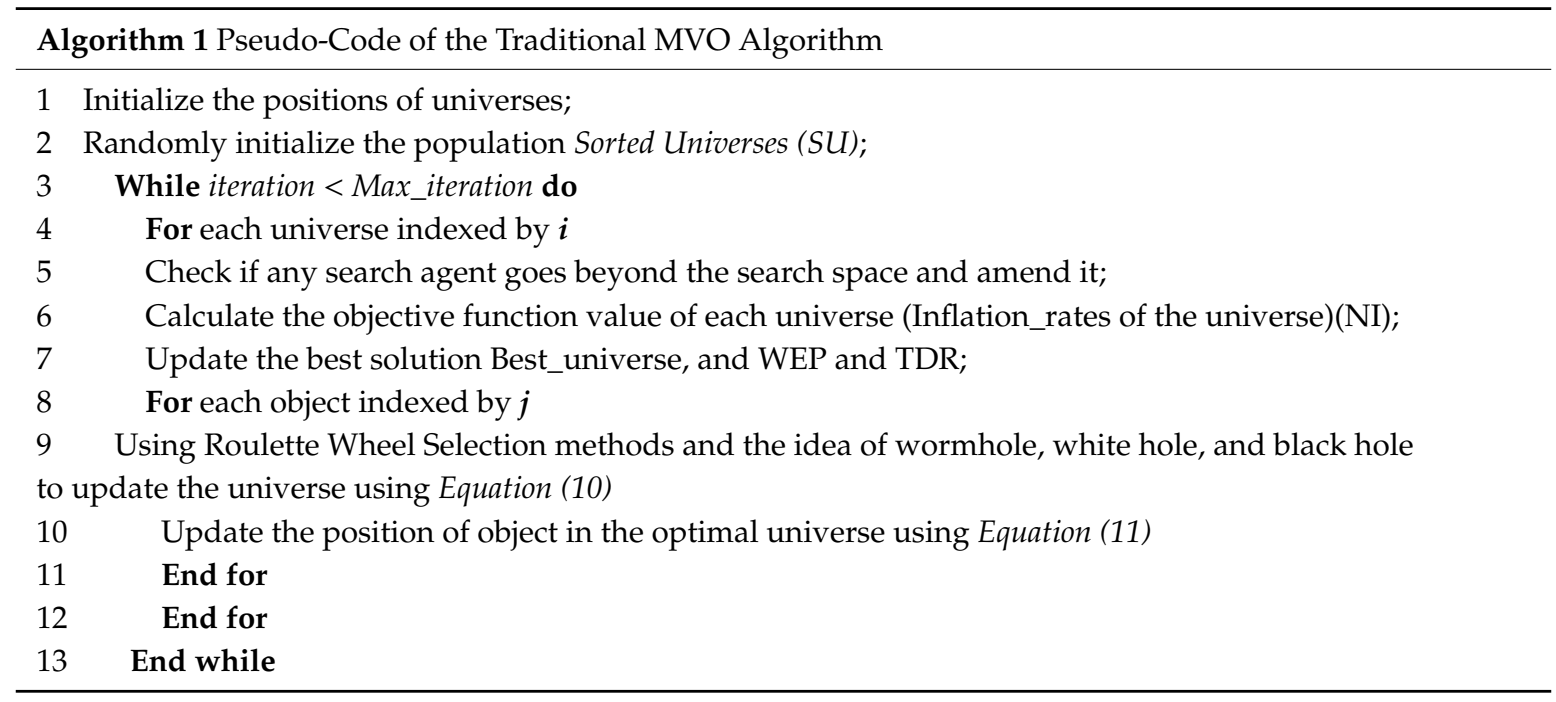

\section{The Proposed Multilevel Thresholding Algorithm}

The idea of MVO optimization algorithm is interesting, which combines physical concepts such as multiverse, wormhole, white hole, black hole and so on. The roulette selection mechanism is utilized in the selection of white holes/black holes, which makes the generation of black holes/white holes in the whole universe very random. Location updates are limited by factors such as current location and object range. Based on the above, in this paper, the roulette selection mechanism was replaced with the tournament selection. Inspired by Cuckoo Search Optimization (CS) $[40,41]$, Flower Pollination Algorithm (FPA) [42,43] and Martingale Algorithm (DA) [44], LMVO has introduced the concept of Lévy flight into white hole/black update. Based on this, a better algorithm model is obtained by adding a mutation factor in this paper [37]. Taking the Masi entropy method as the objective function, through the proposed multiverse optimization algorithm, the maximum value of Masi entropy can be found quickly and stably. Finally, the optimal thresholds $\left(t_{R}, t_{G}, t_{B}\right)$ of three different color components (red, green and blue) in the input color image are determined, so as to achieve a better image segmentation effect.

\subsection{Selection Schemes}

In this section, the selection schemes are described. That is, the roulette selection mechanism of the original algorithm and the tournament selection used to replace the roulette selection mechanism in the algorithm proposed in this paper. Both mechanisms are selected based on fitness function values. Selection strategies are to judge the current individual and determine which individual is used for position update according to the fitness value in the hope of obtaining a higher fitness value in the next iteration. The selection strategy is to judge the current individual and determine which individual to use for location update according to the fitness value. Different selection strategies have different calculation methods of selection, and a more suitable selection mechanism with the algorithm can easily obtain better results to a large extent [45].

\subsubsection{Roulette Wheel Selection}

In roulette wheel selection [46], a roulette wheel is made up of all the individuals selected. The probability of a individual is proportional to its fitness value. That is, the larger the fitness value of a individual is, the greater the number of shares corresponding to that part of the roulette wheel will be. As can be seen from Figure 2, when the wheel stops after rotation, the pointer will randomly select, and the part that accounts for a large number of shares will have a great chance to be selected. 
Of course, we can find that all the parts have the chance to be selected. The probability of selection can be expressed by mathematical formula as:

$$
P_{i}=\frac{f_{i}}{\sum_{j=1}^{n} f_{i}}
$$

where $f_{i}$ indicates the fitness value of the $i$-th position.

The advantage of Roulette is that each individual is likely to be selected. Consequently, the diversity of the population is preserved. However, the selection mechanism of roulette still has some shortcomings:

1. Outstanding individuals will introduce a bias in the beginning of the search that may cause a premature convergence and a loss of diversity.

2. If the fitness values of individuals in a group are very similar, the selection probability of the better and the worse individuals is very close, so it is difficult for the group to develop in a better direction.

3. Many references have proved that this option is not suitable for minimization $[47,48]$.

4. The algorithm procedure of roulette wheel selection depicted in Algorithm 2.

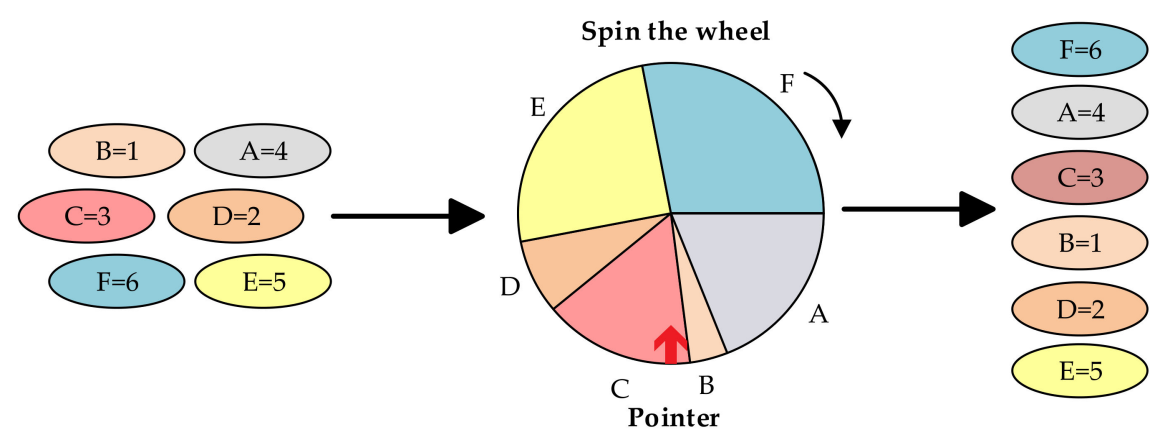

Figure 2. The roulette wheel selection.

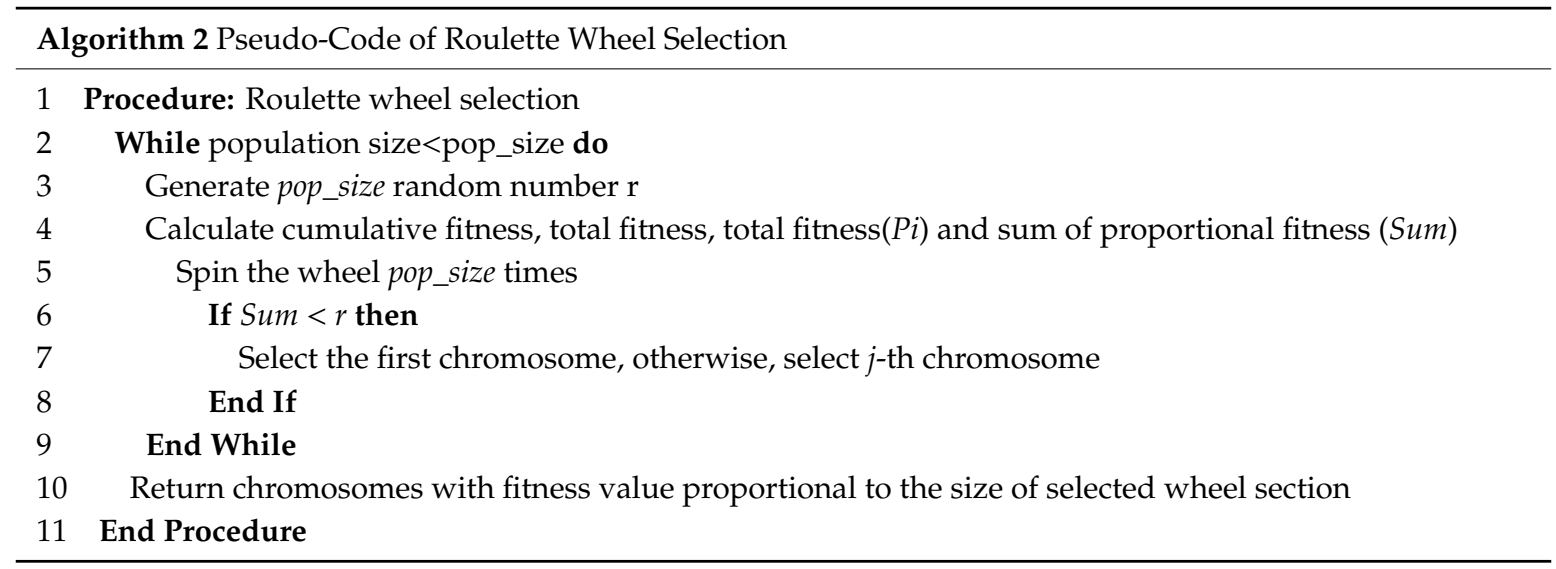

\subsubsection{Tournament Selection}

Tournament selection [49] is a mechanism similar to competition. The concept is quite simple and, with a probability of $68 \%$ in the confidence interval [50], a group of values $(n)$ was randomly selected from the fitness function values (all participants) of all individuals in the population $(N, n \leq N)$. Generate a random number $r, r \in[0,1]$; according to the selection probability, generate the selection pressure $p$. The values in the selected group are compared (the contest), and the optimal value 
(the winner) is determined by the comparison of $r$ and $p$. The optimal value is then substituted into the next iteration. Similarly, competition selection provides all individuals with the opportunity to compete fairly and the diversity of the population is preserved.

Tournament selection has several advantages:

1. Time complexity is more effective;

2. Not susceptible to optimal biasing;

3. No requirement for fitness scaling or sorting [45,48].

However, at the same time, there are also some shortcomings:

1. Suitable for small populations, large populations will lose diversity and fall into local optimum;

2. Relatively, slow convergence speed

Figure 3 illustrates this mechanism. Population size $N$ is set to 8 , and a group is randomly selected to participate in the competition. Membership size $n$ is set to 3. Choose the optimal one through competition.

These two update mechanisms are different in principle. In this newly selected mechanism, a set of better values is selected according to the probability ratio among the existing fitness function values, and each selection is more focused on a better individual. The number of members in the group has a great influence on the optimal value selection, so we set an integer value ranging from 2 to the total number, called the tour parameters [51]. Here, we give the pseudo-code of tournament selection in Algorithm 3.

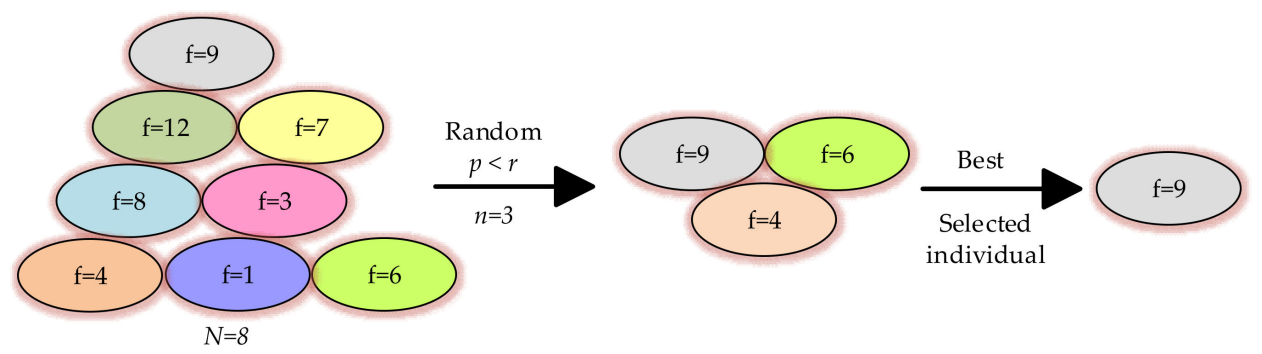

Figure 3. The tournament selection.

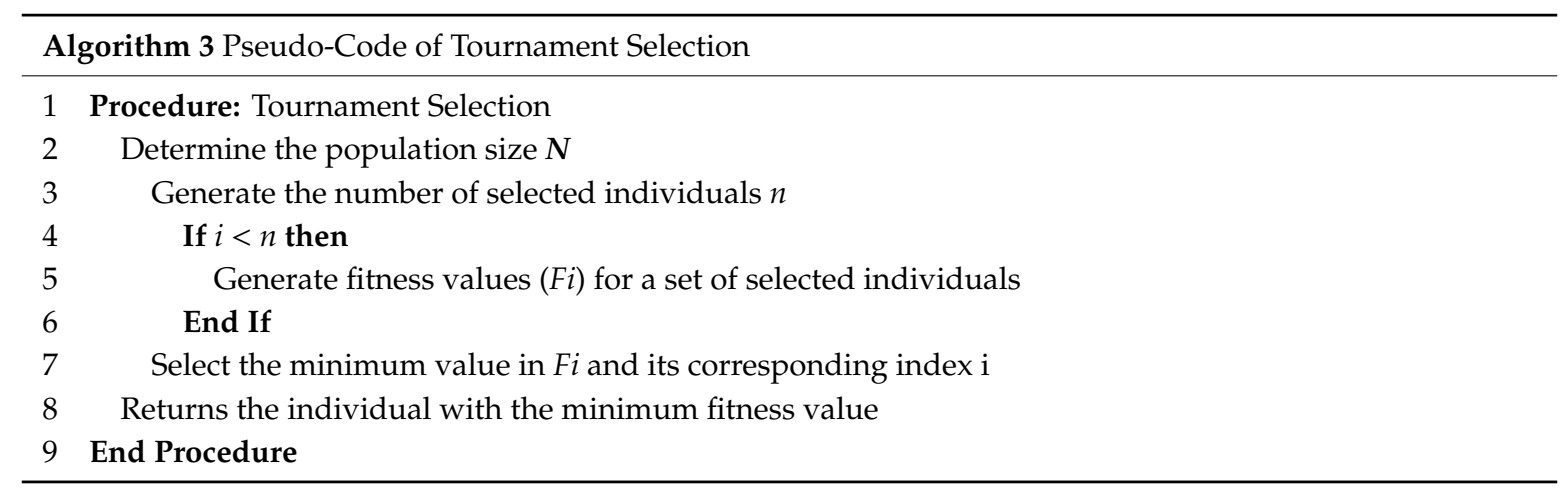

\subsection{Léry Flight}

Lévy flight is very common in nature, which is a kind of mathematical model of rapid flight, rapid jump. It is usually used to describe the behavior of birds, insects and other flying animals [52]. In the past few years, many studies have proved that it has great advantages in improving the convergence speed of the optimization algorithm and the convergence of the global optimal solution. Usually, it is considered as an operator that permits to enhance metaheuristic algorithms [53,54]. The mathematical model can be described as:

$$
\operatorname{Levy}(\lambda)=0.01 \times \frac{\mu \times \sigma}{|v|^{\frac{1}{\beta}}},
$$


where $\mu$ and $v$ obey the normal distribution, $\lambda=\beta+1$ :

$$
\mu \sim N\left(0, \sigma^{2}\right), v \sim N\left(0, \sigma_{v}^{2}\right)
$$

with

$$
\sigma=\left(\frac{\Gamma(1+\beta) \times \sin \left(\frac{\pi \beta}{2}\right)}{\Gamma\left(\frac{1+\beta}{2}\right) \times \beta \times 2^{\left(\frac{\beta-1}{2}\right)}}\right)^{\frac{1}{\beta}}, \sigma_{v}=1 .
$$

The step length $s$ can be expressed as:

$$
s=\frac{\mu}{|v|^{\frac{1}{\beta}}} .
$$

With the change of controlling parameter $\beta$, the shape of probability density function will also change, which will affect the shape of the tail region. Here, $\beta$ is a constant, $\beta=1.5$.

The selection strategy used in this paper, tournament selection, has the disadvantage of slow convergence. Lévy flight can be used as an operator for optimization improvement. In terms of global optimization of the algorithm, Lévy flight's occasional large leap can effectively avoid falling into local optimization.

\subsection{Tournament-Based Lévy Multiverse Optimization Algorithm}

In this paper, the MVO algorithm is improved by changing selection schemes, adding the random walk strategy and improving the updating formula, being aimed at improving the wide adaptability of the MVO algorithm to high dimensional multimodal optimization problems.

Tournament selection is the most effective when dealing with minimization issues [34,35]. In this regard, we invert the fitness value in the code for tournament selection. Adding the competition mechanism in the universe to better cooperate with black holes and white holes for material renewal between the universe. More effectively, the maximum fitness function value is screened out for the updating of the next generation.

For the location update of the optimal universe, we made several additional optimization improvements. Firstly, the current position is changed to the local optimal position. Secondly, taking the advantage of cuckoo algorithm in position updating, two arbitrary positions in any universe are randomly selected to make a difference (mutation factor). The diversity of the population would decline sharply in the later period. The introduction of this mutation factor and Lévy 's random walk strategy improves the development ability and maintains the diversity of the population [55].

The improved position update formula can be expressed as:

$$
x_{i+1}^{j}= \begin{cases}\left\{x_{\text {Best }}^{j}+\left\{x_{a}-x_{b} \times r_{3}+T D R \times\left\{\left\{u b_{j}-l b_{j} \times L e v y+l b_{j}\right.\right.\right.\right. & r_{2}<W E P \\ x_{i}^{j} & r_{2} \geq W E P^{\prime}\end{cases}
$$

where $x_{B e s t}^{j}$ represents the current optimal value, $x_{a}, x_{b}$ indicate the position of two different objects in the universe, respectively:

$$
T D R \times\left(\left(u b_{j}-l b_{j}\right)+l b_{j}\right) .
$$

This part has not changed much because this idea ensures that individuals can get random positions in the search space. In addition, the pseudo-code of the proposed algorithm shows in Algorithm 4 . 


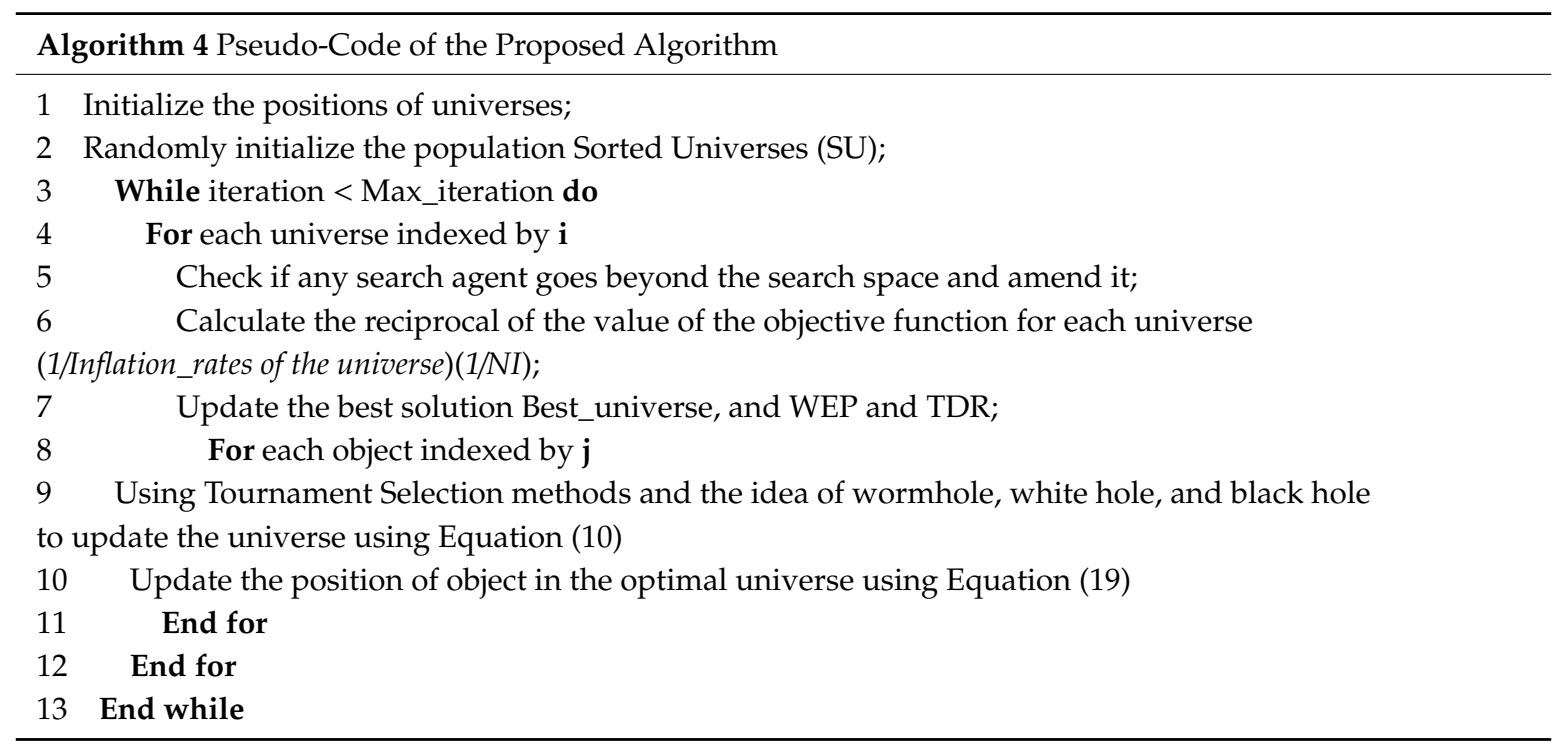

\subsection{The Proposed TLMVO-Based Multilevel Thresholding Method}

Combining the TLMVO algorithm with a multilevel threshold method, Masi entropy is taken as the objective function. By determining the maximum entropy between classes, the corresponding optimal threshold is obtained, so as to obtain better image segmentation results. The position of the individual is determined by multiple thresholds, and different individuals make up the universe. The inflation rate of universe corresponds to the value of the objective function, thus establishing the relationship between the optimization algorithm and segmentation function.

From the overall perspective of the program, the individual and other related parameters are initialized, calculate the initial fitness function values with formulas Equations (6) and (7). The fitness function value is screened through the tournament selection mechanism, determine the optimal individual, and exchange individuals between the universe. Under the triple constraint of individual range Equation (20), wormhole existence rate (WEP) Equation (12), travel distance rate (TDR) Equation (13) and Lévy Flight Equation (15), the optimal universe was updated with formula Equation (20). The iterative loop determines the optimal threshold and completes the image segmentation. The overall flow chart is shown in Figure 4.

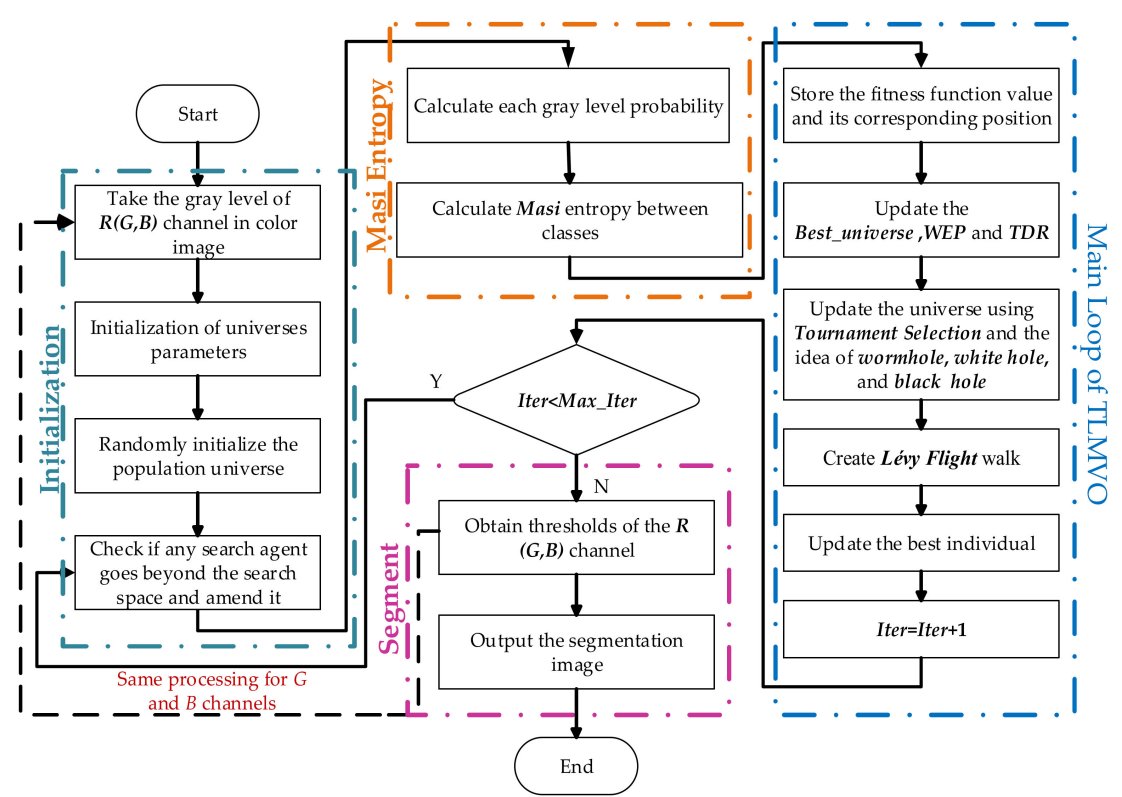

Figure 4. The flow chart of the TLMVO-based multilevel thresholding method. 


\section{The Computational Experiments and Results}

In order to evaluate the performance of the algorithm, computational experiments were carried out on the convergence curve of the segmentation function and the evaluation index of image segmentation effect. The general structure is as follows: Section 5.1 briefly introduces the basic experimental environment; the measured images, comparison algorithm and related parameters are given in Section 5.2; in Section 5.3, several performance measures are chosen to evaluate the segmentation effect; the experimental data are analyzed in Section 5.4 finally.

\subsection{Experimental Setup}

The computer is configured with Intel(R) Pentium(R) CPU G4560@3.50 GHz (Intel, Santa Clara, CA, USA), Microsoft Windows 7 system (Microsoft, Redmond, WA, USA), and the operating environment is Matlab R2017b (The MathWorks Inc., Natick, MA, USA).

The proposed method is compared with several well-known metaheuristicss algorithms. Each of them contains different characteristics, including

1. The traditional MVO algorithm [30];

2. The state-of-the-art LMVO algorithm [37];

3. An interesting bionic algorithm named ant lion algorithm (ALO) which can always find the maximum in the latest metaheuristics algorithm [56];

4. A new complex swarm intelligent optimization technology, dragonfly algorithm (DA) [43];

5. FPA, inspired by the process of flower pollination of flowering plants in nature which is simple and requires fewer parameters to be adjusted [41,42];

6. An earlier proposed evolutionary algorithm, PSO [57-60];

7. CS which is based on the brood parasitism of some cuckoo species, along with Lévy flights' random walks $[39,40]$.

The seven comparison algorithms correspond to four relatively novel algorithms and three relatively basic algorithms, respectively. The parameters of these algorithms are selected from the references related to image segmentation, which are shown in Table 1.

Table 1. Parameters of algorithms.

\begin{tabular}{cccc}
\hline Reference & Algorithm & Parameters & Value \\
\hline \multirow{2}{*}[30]{} & \multirow{2}{*}{ MVO $^{1}$} & Mining capability $p$ & $1 / 6$ \\
& & Random parameters $r_{1}, r_{2}, r_{3}, r_{4}$ & {$[0,1]$} \\
& LMVO $^{1}$ & Contrast parameter $H$ & 0.5 \\
\hline \multirow{2}{*}[37]{} & TLMO' $^{1}$ & Selection pressure $p$ & 1.5 \\
& ALO & Screening probability $r$ & {$[0,1]$} \\
& & Switch possibility & {$[0,1]$} \\
\hline$[61]$ & & Inertial weight & 0.5 \\
\hline \multirow{2}{*}[44]{} & DA & Seperation weight & {$[0.5,0.9]$} \\
& & Alignment weight & {$[0,0.2]$} \\
& & Cohesion weight & \\
\cline { 3 - 4 } & & Maximum velocity & 25.5 \\
& & Food attraction weight & {$[0,2]$} \\
& & Enemy distraction weight & {$[0,0.1]$} \\
\hline \multirow{2}{*}[43]{} & FPA & Switch possibility & 0.4 \\
& & Lévy controlling constant $\beta$ & 1.5 \\
\hline
\end{tabular}


Table 1. Cont.

\begin{tabular}{cccc}
\hline Reference & Algorithm & Parameters & Value \\
\hline \multirow{3}{*}[59]{} & & Maximum inertia weight & 0.9 \\
& \multirow{3}{*}{ PSO } & Minimum inertia weight & 0.4 \\
& & Learning factors $c_{1}$ and $c_{2}$ & 2 \\
& & Maximum velocities & +120 \\
& & Minimum velocities & -120 \\
\hline \multirow{2}{*}[7]{} & \multirow{2}{*}{ CS } & Mutation probability value $P_{a}$ & 0.25 \\
& & Scale factor $\beta$ & 1.5 \\
\hline
\end{tabular}

${ }^{1}$ As an improvement of the algorithm, the same parameters are not given repeatedly.

Comparative experiments were conducted using control variable method. The maximum number of iterations for all algorithms is 500 and the number of population size is 25 . For each image, each algorithm runs 30 times separately. The threshold dimension of $\mathrm{K}$ is divided into high dimension $(\mathrm{K}=10,12)$ and low dimension $(\mathrm{K}=4,6,8)$.

\subsection{Satellite Color Image Used}

This paper presents a new improved TLMVO algorithm for satellite image segmentation using Masi entropy. In satellite images, there are different bands and different wavelength areas. The processing of satellite images is carried out in different wavebands (the full band combination specifications are presented in Table 2. Secondly, the image features are very dense and the information from one area to another changes rapidly. At the same time, satellite images are generally of high resolution. All these will affect the efficiency of the algorithm, which will lead to inefficiency of the algorithm and increase the amount of computation in segmentation. Therefore, the accurate segmentation of satellite images is a very challenging task [5].

Table 2. Characteristics and Use [4].

\begin{tabular}{cccc}
\hline Band No. & Name & Wavelength $(\mu \mathrm{m})$ & Characteristics and Use \\
\hline 1 & Visible blue & $0.45-0.52$ & Maximum water penetration \\
2 & Visible green & $0.52-0.60$ & Good for measuring plant Vigor \\
3 & Visible red & $0.63-0.69$ & Vegetation discrimination \\
4 & Near infrared & $0.76-0.90$ & Biomass and shoreline Mapping \\
5 & Middle Infrared & $1.55-1.75$ & Moisture content of soil \\
6 & Thermal Infrared & $10.4-12.5$ & Soil moisture, thermal mapping \\
7 & Middle Infrared & $2.08-2.35$ & Mineral mapping \\
\hline
\end{tabular}

Ten satellite images are selected for segmentation to achieve better contrast effect. Each threshold has a range of $[0,256)$, and thus the search space is $[0,256)^{25}$. The size and histogram of each satellite image are presented in Figure 5, which are from the aerial data set [62]. For each color image and threshold level, 30 independent running experiments were conducted [63,64]. The corresponding thresholds for each optimal solution are reported in Table 3.
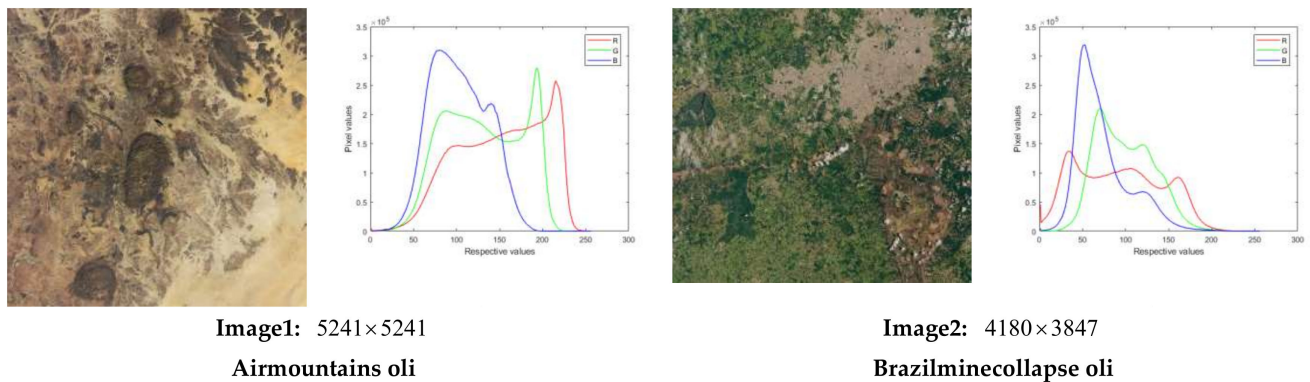

Airmountains oli

Image2: $4180 \times 3847$

Brazilminecollapse oli

Figure 5. Cont. 


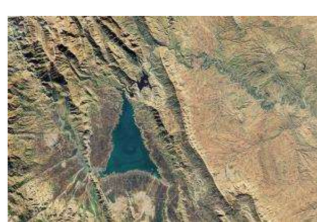

(a)

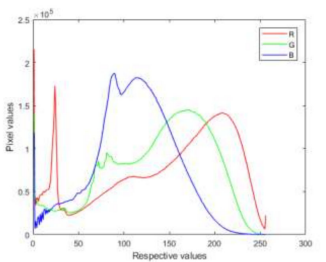

(b)

Image3: $5339 \times 3559$

The Lake Dukan situated in the Zagros Mountains

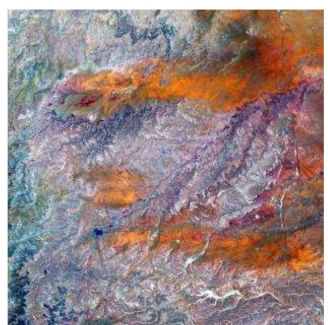

(a)

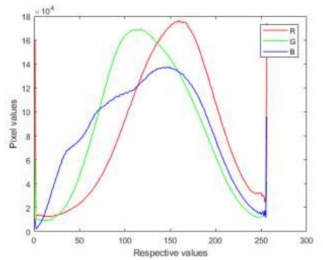

(b)

Image5: $4627 \times 4627$

Gaara oli

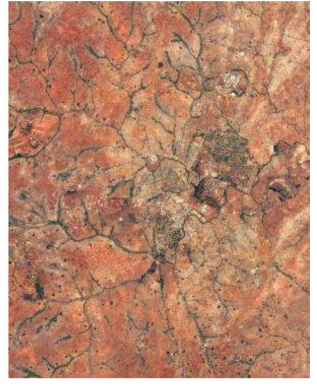

(a)

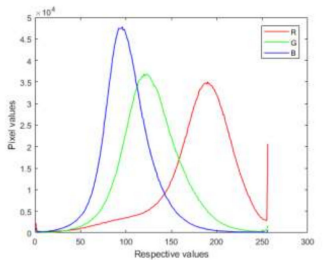

(b)

Image7: $1440 \times 1800$

The Malawian capital of Lilongwe

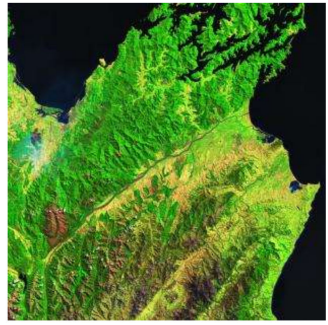

(a)

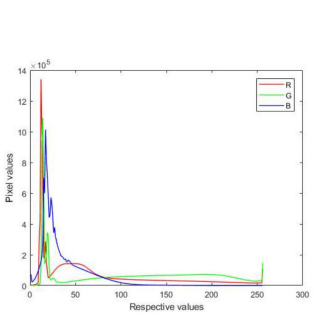

(b)

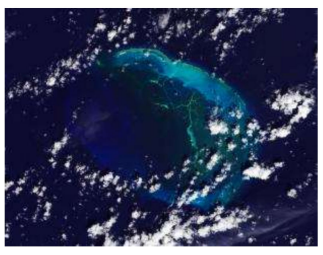

(a)

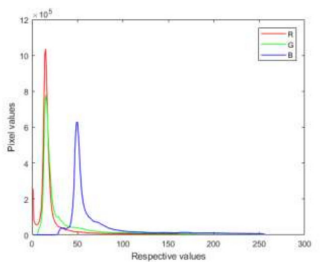

(b)

Image4: $3601 \times 2742$

Eastisland oli

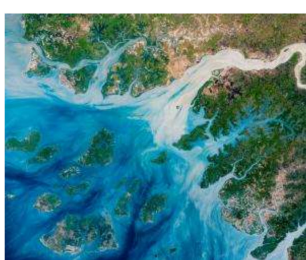

(a)

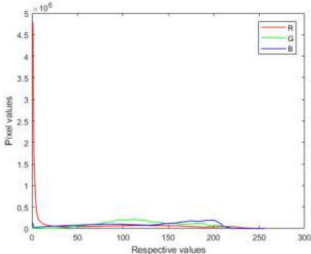

(b)

Image6: $5130 \times 4203$

The Meandering Estuaries of Guinea - Bissau

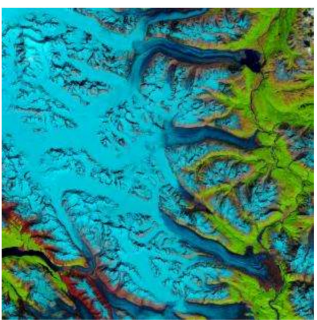

(a)

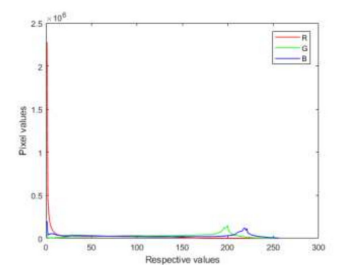

(b)

Image8: $2718 \times 2718$

Lowell msi

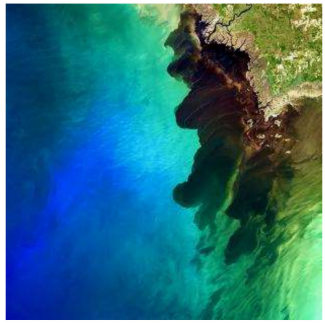

(a)

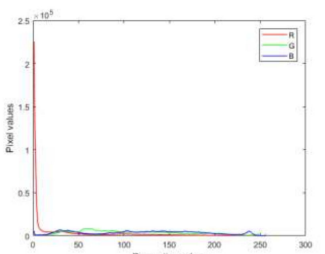

(b) Image9: $4096 \times 4096$ Newzealand oli

Image10: $936 \times 936$

A Blackwater River Meets the Sea

Figure 5. The experimental satellite images and corresponding histogram images. 
Table 3. Optimal solution for each algorithm under Masi entropy

\begin{tabular}{|c|c|c|c|c|c|c|c|c|c|c|c|c|c|c|c|c|c|c|c|c|c|c|c|c|c|}
\hline \multirow{2}{*}{$\begin{array}{c}\text { TEST } \\
\text { IMAGES }\end{array}$} & \multirow{2}{*}{ K } & \multicolumn{3}{|c|}{ TLMVO } & \multicolumn{3}{|c|}{ LMVO } & \multicolumn{3}{|c|}{ MVO } & \multicolumn{3}{|c|}{ ALO } & \multicolumn{3}{|c|}{ DA } & \multicolumn{3}{|c|}{ FPA } & \multicolumn{3}{|c|}{ PSO } & \multicolumn{3}{|c|}{ CS } \\
\hline & & $\mathbf{R}$ & G & B & $\mathbf{R}$ & G & B & $\mathbf{R}$ & G & B & $\mathbf{R}$ & G & B & $\mathbf{R}$ & G & B & $\mathbf{R}$ & G & B & $\mathbf{R}$ & G & B & $\mathbf{R}$ & G & B \\
\hline \multirow{20}{*}{1} & & 57100 & 5093 & 4383 & 57100 & 5698 & 4383 & 57100 & 5698 & 4383 & 57100 & 5698 & 4383 & 57103 & 5698 & 4381 & 71119 & 5069 & 4684 & 57100 & 5698 & 4383 & 5493 & 48103 & 4791 \\
\hline & 4 & 140182 & 131168 & 121158 & 140182 & 138173 & 122163 & 143181 & 138173 & 121158 & 140182 & 138173 & 122163 & 142184 & 138173 & 121158 & 170205 & 106158 & 115164 & 140182 & 138173 & 122163 & 138183 & 138180 & 117166 \\
\hline & & 5780 & 5081 & 4374 & 5576 & 4765 & 3857 & 5780 & 4664 & 3857 & 5780 & 5081 & 3857 & 5782 & 4664 & 3857 & 6072 & 425794 & 2260 & 5780 & 4664 & 4063 & 5499 & 5490 & 3983 \\
\hline & 6 & 111142 & 112144 & 103130 & 107139 & 99134 & 88121 & 111141 & 93122 & 85112 & 111142 & 112144 & 85113 & 114144 & 93122 & 85112 & 88132 & 115128 & 107124 & 111142 & 93123 & 90117 & 117139 & 114140 & 104117 \\
\hline & & 172202 & 175205 & 163229 & 172202 & 166203 & 152171 & 172202 & 151180 & 139166 & 172202 & 175205 & 140166 & 175203 & 152180 & 139166 & 142169 & 157 & 143171 & 172202 & 152180 & 143166 & 169186 & 165181 & 140172 \\
\hline & & 5470 & 3750 & 3855 & 4664 & 4661 & 3849 & 5572 & 4659 & 3852 & 4664 & 4663 & 3853 & 5470 & 4357 & 3853 & 4751 & 354354 & 4046 & 4664 & 4661 & 3853 & 4460 & 4363 & 4661 \\
\hline & 8 & & & & & & & 94117 & & & 84108 & & & 94118 & & & & 6693 & & & & & & & 81102 \\
\hline & 0 & 145172 & 114138 & 123148 & 127150 & 131155 & 109130 & 139162 & 127152 & 112133 & 132156 & 134157 & 113134 & 141164 & 120155 & 116136 & 120147 & 122151 & 106135 & 131156 & 134158 & 114134 & 132160 & 130140 & 113125 \\
\hline & & 200227 & 160183 & 167230 & 177203 & 180205 & 152171 & 184206 & 180205 & 154171 & 180206 & 181205 & 155171 & 186208 & 180205 & 155171 & 191212 & 182 & 157214 & 180205 & 182205 & 155171 & 194210 & 160200 & 135161 \\
\hline & & 4657 & 4156 & 3852 & 4663 & 3750 & 3346 & 4663 & 4156 & 3346 & 4664 & 4156 & 3346 & 5572 & 4156 & 3849 & 3953 & 405071 & 2540 & 4664 & 4156 & 3346 & 4454 & 3952 & 3039 \\
\hline & & 681 & 7180 & 6885 & 79.97 & 6382 & 6485 & 7793 & & 6077 & 8097 & 71 (19) & 5874 & 91112 & 7088 & 6580 & 8197 & 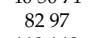 & 5160 & 82104 & 17100 & 5974 & 600 & 6177 & 6673 \\
\hline & 10 & & 107125 & 103120 & 115133 & 101120 & 107129 & 112131 & 102118 & 94110 & 115133 & 108127 & 91107 & 132151 & & & & 119140 & & & & & & & \\
\hline & 0 & 135156 & 144163 & 137155 & 152171 & 140161 & 154171 & 151170 & 136158 & 123138 & 151171 & 145164 & 124141 & 171189 & 137160 & 126141 & 127160 & 154190 & 101127 & 167187 & 144163 & 122139 & 157168 & 126135 & 123136 \\
\hline & & 179205 & 183205 & 171184 & 189208 & 182205 & 206208 & 189208 & 181205 & 155171 & 191210 & 183205 & 156171 & 207227 & 183206 & 156171 & 188206 & 227 & 147176 & 206227 & 184205 & 155171 & 193210 & 159167 & 146164 \\
\hline & & 4657 & 4156 & 2333 & 4663 & 3747 & 3346 & 4663 & 3750 & 3040 & 4657 & 3748 & 3343 & 4657 & 4356 & 3043 & 6474 & & 1930 & 4657 & 3750 & 13344 & 4248 & 4753 & 3945 \\
\hline & & & & & & & & & & & & & & & & & & & & & & & & & \\
\hline & & 99115 & 100116 & 6276 & 105122 & 8499 & 8498 & 105119 & & 7792 & 116136 & & & & & & & & & & & & & & \\
\hline & 12 & 132149 & 132148 & 90106 & 139155 & 115132 & 112126 & & & & & & & & & & & 50 & & & & & & & \\
\hline & & & & 122138 & & & & & & & & & & & & & & & & & & & & & \\
\hline & & 205227 & 197207 & 155171 & 207227 & 184205 & 171184 & 208227 & 188205 & 163173 & 212227 & 187205 & 156171 & 210227 & 197207 & 163173 & 182197 & & 168183 & 227256 & 186205 & 171 & 190205 & 174189 & 167171 \\
\hline & 4 & $\begin{array}{c}60117 \\
174197\end{array}$ & $\begin{array}{c}3880 \\
123170\end{array}$ & $\begin{array}{c}\begin{array}{c}3388 \\
141\end{array} 170\end{array}$ & $\begin{array}{c}60117 \\
174197\end{array}$ & $\begin{array}{c}59117 \\
157187\end{array}$ & $\begin{array}{c}3388 \\
141170\end{array}$ & $\begin{array}{r}5088 \\
126182\end{array}$ & $\begin{array}{l}38111 \\
157187\end{array}$ & $\begin{array}{c}4489 \\
141170\end{array}$ & $\begin{array}{r}5088 \\
126182\end{array}$ & $\begin{array}{r}59117 \\
157187\end{array}$ & $\begin{array}{c}3388 \\
141170\end{array}$ & $\begin{array}{r}5089 \\
126188\end{array}$ & $\begin{array}{r}63117 \\
157187\end{array}$ & $\begin{array}{r}3388 \\
141177\end{array}$ & $\begin{array}{c}6784 \\
116195\end{array}$ & $\begin{array}{r}64115 \\
164211\end{array}$ & $\begin{array}{l}5890 \\
138178\end{array}$ & $\begin{array}{c}5895 \\
138178\end{array}$ & $\begin{array}{l}38111 \\
157187\end{array}$ & $\begin{array}{c}3388 \\
141170\end{array}$ & $\begin{array}{c}4177 \\
120179\end{array}$ & $\begin{array}{l}58117 \\
157184\end{array}$ & $\begin{array}{l}5194 \\
18187\end{array}$ \\
\hline & & $\begin{array}{c}174197 \\
3061\end{array}$ & $\frac{123170}{3863}$ & & & $\begin{array}{c}157187 \\
3861\end{array}$ & & & & & & & & & & & & & $\begin{array}{r}138178 \\
3691\end{array}$ & $\begin{array}{r}138178 \\
3068\end{array}$ & $\begin{array}{r}157187 \\
3863\end{array}$ & $\frac{141170}{1946}$ & 120179 & 157184 & 148187 \\
\hline & 6 & 94127 & & 881. & $\begin{array}{l}4311 \\
121 \\
172\end{array}$ & $\begin{array}{l}901123 \\
91\end{array}$ & 881 & & 158 & $\begin{array}{l}19 \\
821\end{array}$ & $\begin{array}{r}356 \\
1061\end{array}$ & $\begin{array}{l}386 \\
931\end{array}$ & $\begin{array}{l}19 . \\
821\end{array}$ & $\begin{array}{r}421 \\
1121\end{array}$ & $\begin{array}{l}386 \\
951\end{array}$ & $\begin{array}{l}275 \\
931\end{array}$ & $\begin{array}{l}5180 \\
97135\end{array}$ & & $\begin{array}{r}3691 \\
116148\end{array}$ & $\begin{array}{c}3068 \\
106138\end{array}$ & $\begin{array}{l}3863 \\
93124\end{array}$ & $\begin{array}{r}1946 \\
88135\end{array}$ & $\begin{array}{c}414143 \\
114142\end{array}$ & $\begin{array}{l}3342 \\
77122\end{array}$ & \\
\hline & & 174197 & 158187 & 158193 & 190212 & 157187 & 157181 & 174197 & 187 & 141170 & 174197 & 158187 & 141170 & 177205 & 160189 & 168193 & 176186 & 191214 & 173185 & $\begin{array}{l}177205 \\
\end{array}$ & 160189 & 157181 & $\begin{array}{l}173191 \\
\end{array}$ & 162194 & 169187 \\
\hline & & & & & & & & & & & & & & & & & & & & & & & & & \\
\hline & & & & & & & & & & & & & & & & & & 105 & & & & & & & \\
\hline & 8 & 141172 & 127155 & 135157 & 120147 & 128157 & 135157 & 120146 & & 108136 & 14517 & & 1091 & 146172 & 130157 & 133149 & & 1662 & 90139 & 144172 & & & & 138160 & 127144 \\
\hline & & 190212 & 174202 & 177197 & 177205 & 179208 & 177203 & 177205 & 174202 & 157181 & 190212 & 177208 & 157185 & 190212 & 179208 & 170193 & 186212 & 211 & 160195 & 190212 & 177208 & 170197 & 170190 & 178203 & 161194 \\
\hline & & 1737 & 1737 & & & 3244 & 1732 & & 3652 & & 1737 & & & & & & & 68 & 2533 & & & 1733 & & 2336 & \\
\hline & & & & & & & & & & & & & & & & & & & & & & & & & \\
\hline & 10 & 105126 & 105126 & 102128 & 106126 & 101125 & 93113 & 103126 & 104121 & & 10512 & 1081 & & 102128 & & & & & & & & & & & \\
\hline & & & & & & & 136157 & & & & & & & & & & & & & & & & & & \\
\hline & & 190212 & 190212 & 178203 & 190212 & 187208 & 177203 & 190212 & 177202 & 170193 & 190212 & 187208 & 178203 & 178203 & 208224 & 178203 & 187216 & 196 & 184210 & 190212 & 189208 & 170193 & 202210 & 208220 & 172197 \\
\hline & & & & & 92236 & 2638 & & & & 1115 & 1735 & 324 & 173 & 173 & & 172 & 54 & 3272 & & & & & 1954 & 173 & \\
\hline & & & & & & & & & & & & & & & & & & & & & & & & & \\
\hline & & & & & 97121 & 8310 & 821 & 8710 & 86104 & & 931 & 95113 & & & 97119 & & & 1261 & & 84102 & & & 109118 & 901 & 7783 \\
\hline & 12 & 126149 & 1321 & 1281 & 145169 & 1191 & 127141 & 123139 & 121136 & 1111 & 1321 & 1301 & 113131 & 124138 & 1391 & 116133 & 146173 & 171174 & 112119 & 120137 & 120137 & 115134 & 135175 & 125138 & 102116 \\
\hline & & 174190 & 169187 & 157175 & 182197 & 1541 & 157172 & 153172 & 153168 & 14516 & 170182 & 160174 & 1441 & 1531 & 17018 & & 197221 & 182209 & 137 & 154174 & 155170 & 149170 & 184200 & 160168 & 131 \\
\hline & & 212 & 202218 & 193210 & 212 & 187208 & 191208 & 190212 & 187208 & 181203 & 197212 & 189210 & 179203 & 190212 & 205220 & 179203 & 239 & 227 & 167191 & 190212 & 189210 & 197256 & 212237 & 175194 & 157166 \\
\hline
\end{tabular}


Table 3. Cont

\begin{tabular}{|c|c|c|c|c|c|c|c|c|c|c|c|c|c|c|c|c|c|c|c|c|c|c|c|c|c|}
\hline \multirow{2}{*}{$\begin{array}{c}\text { TEST } \\
\text { IMAGES }\end{array}$} & \multirow{2}{*}{ K } & \multicolumn{3}{|c|}{ TLMVO } & \multicolumn{3}{|c|}{ LMVO } & \multicolumn{3}{|c|}{ MVO } & \multicolumn{3}{|c|}{ ALO } & \multicolumn{3}{|c|}{ DA } & \multicolumn{3}{|c|}{ FPA } & \multicolumn{3}{|c|}{ PSO } & \multicolumn{3}{|c|}{ CS } \\
\hline & & $\mathbf{R}$ & G & B & $\mathbf{R}$ & G & B & $\mathbf{R}$ & G & B & $\mathbf{R}$ & G & B & $\mathbf{R}$ & G & B & $\mathbf{R}$ & G & B & $\mathbf{R}$ & G & B & $\mathbf{R}$ & G & B \\
\hline \multirow{18}{*}{3} & 4 & $\begin{array}{r}69109 \\
158212\end{array}$ & $\begin{array}{r}5596 \\
137185\end{array}$ & $\begin{array}{c}3668 \\
108156\end{array}$ & $\begin{array}{r}69109 \\
158212\end{array}$ & $\begin{array}{r}3063 \\
121185\end{array}$ & $\begin{array}{r}59102 \\
144176\end{array}$ & $\begin{array}{r}69109 \\
158212\end{array}$ & $\begin{array}{r}5594 \\
136185\end{array}$ & $\begin{array}{c}5799 \\
144176\end{array}$ & $\begin{array}{r}75117 \\
163212\end{array}$ & $\begin{array}{r}5596 \\
137185\end{array}$ & $\begin{array}{c}4378 \\
144176\end{array}$ & $\begin{array}{c}72114 \\
162212\end{array}$ & $\begin{array}{r}59100 \\
140185\end{array}$ & $\begin{array}{r}59102 \\
144176\end{array}$ & $\begin{array}{c}70126 \\
168208\end{array}$ & $\begin{array}{r}2661 \\
122201\end{array}$ & $\begin{array}{r}5476 \\
137169\end{array}$ & 75117 & $\begin{array}{r}5999 \\
5895\end{array}$ & $\begin{array}{c}4378 \\
141176\end{array}$ & $\begin{array}{r}79130 \\
765217\end{array}$ & $\begin{array}{r}58103 \\
18182\end{array}$ & $\begin{array}{r}6193 \\
\end{array}$ \\
\hline & & 3668 & 5587 & 2654 & 3668 & 3063 & 3057 & 3668 & 3062 & 3667 & 3668 & 3063 & 2657 & 4075 & 3363 & 3667 & 1984 & 516981 & 2763 & 3668 & 3063 & 3259 & 5075 & 2758 & 4272 \\
\hline & 6 & 100137 & 119151 & 79112 & 100137 & 100136 & 81113 & 100135 & 96132 & 96124 & 100137 & 100136 & 81113 & 109145 & 100136 & 96124 & 100155 & 124159 & 89122 & 100137 & 99134 & 83114 & 125153 & 72100 & 93115 \\
\hline & & 174214 & 185213 & 148176 & 174214 & 173204 & 148176 & 173214 & 170198 & 151177 & 174214 & 175204 & 148176 & 182217 & 173204 & 151177 & 180213 & 194 & 140170 & 174214 & 170198 & 148176 & 186225 & 135186 & 135177 \\
\hline & & 1639 & 1940 & 1939 & 3462 & 1940 & 2447 & 3562 & 3061 & 2446 & 3565 & 3061 & 2447 & 1640 & 3061 & 2549 & 1476 & 93752 & 2754 & 1643 & 3061 & 2446 & 1255 & 2368 & 4471 \\
\hline & 8 & & & & & & & 87117 & & & 93121 & 87112 & & 70100 & & & & 81104 & & & & & & 104137 & 110122 \\
\hline & . & 130161 & 121149 & $\begin{array}{l}104129 \\
1531777\end{array}$ & 140170 & 121151 & 116141 & 146176 & 138165 & 116141 & 150179 & 138165 & $\begin{array}{l}117141 \\
162181\end{array}$ & 135169 & 138165 & 119143 & 164178 & 136182 & 106130 & 139172 & 142170 & 116141 & 148175 & 153171 & 137149 \\
\hline & & 193221 & & 153177 & 206231 & 185213 & 163181 & 209231 & 189213 & 163181 & 209231 & 189213 & 163181 & 206231 & 189213 & 163181 & 202232 & 203 & 142192 & 206231 & 192213 & 163181 & 205223 & 197214 & 165177 \\
\hline & & 1635 & 18361 & 1937 & 1635 & 1838 & 1532 & 1635 & 1638 & 1936 & 1635 & 1839 & 1939 & 1639 & 1943 & 1937 & 4465 & 224261 & 2857 & 1636 & 1838 & 1939 & 1531 & 3363 & 4460 \\
\hline & 10 & $\begin{array}{c}6285 \\
107131\end{array}$ & $\begin{array}{l}80102 \\
124146\end{array}$ & $\begin{array}{l}5774 \\
94113\end{array}$ & $\begin{array}{c}6285 \\
109134\end{array}$ & $\begin{array}{c}6079 \\
100122\end{array}$ & $\begin{array}{l}5168 \\
85104\end{array}$ & $\begin{array}{c}6183 \\
104130\end{array}$ & $\begin{array}{r}6081 \\
102125\end{array}$ & $\begin{array}{l}5071 \\
90109\end{array}$ & $\begin{array}{c}6286 \\
110134\end{array}$ & $\begin{array}{c}6283 \\
105126\end{array}$ & $\begin{array}{l}5976 \\
96115\end{array}$ & & & $\begin{array}{l}5774 \\
93113\end{array}$ & & & & & & & & & \\
\hline & & 156183 & $\begin{array}{l}170192 \\
170\end{array}$ & 133151 & 160186 & $\begin{array}{l}100122 \\
146\end{array}$ & $\begin{array}{l}85104 \\
124144\end{array}$ & 157183 & $\begin{array}{l}1021125 \\
147170\end{array}$ & $\begin{array}{l}90109 \\
129148\end{array}$ & 161187 & $\begin{array}{l}105126 \\
149173\end{array}$ & $\begin{array}{l}96115 \\
134151\end{array}$ & $\begin{array}{l}171147 \\
173198\end{array}$ & $\begin{array}{l}147188 \\
1492\end{array}$ & $\begin{array}{r}93113 \\
132150\end{array}$ & $\begin{array}{r}98834 \\
176202\end{array}$ & 142176 & $\begin{array}{l}97110 \\
133140\end{array}$ & $\begin{array}{l}110136 \\
162187\end{array}$ & $\begin{array}{l}104126 \\
148171\end{array}$ & $\begin{array}{r}966115 \\
134151\end{array}$ & $\begin{array}{l}119151 \\
162173\end{array}$ & $\begin{array}{l}134146 \\
159193\end{array}$ & $\begin{array}{l}106117 \\
132145\end{array}$ \\
\hline & & 212232 & 213 & 168183 & 212232 & 192213 & 163181 & 210231 & 192213 & 168183 & 212232 & 192213 & 168184 & 221239 & 192213 & 168184 & 217232 & 201 & 147193 & 212232 & 192213 & 168184 & 202222 & 210219 & 161180 \\
\hline & & 1632 & 1838 & 1835 & 1632 & 1430 & 1529 & 1630 & 1836 & 1532 & 1634 & 1838 & 1936 & 1634 & 1634 & 1938 & 1731 & & 2632 & 1634 & 1838 & 1936 & 1738 & 3445 & 2135 \\
\hline & & & & & & & & & & & & & & & & & & & & & & & & & \\
\hline & & 86106 & 93110 & 8399 & & & & 83100 & & & & & & & & & & & & & & & & & \\
\hline & 12 & 125145 & 127144 & 115131 & & 113131 & & & & & & & & & & & & & & & & & & & \\
\hline & & & & & & & & & & & & & & & & & & & & & & & & & \\
\hline & & 212231 & 196213 & 176187 & 212231 & 192213 & 183246 & 210231 & 196213 & 176187 & 221239 & 204218 & 184237 & 222239 & 204218 & 176187 & 232240 & 69192 & 181191 & 221237 & 204218 & 176187 & 219240 & 191219 & 157177 \\
\hline & 4 & 3580 & 3683 & 4077 & 3580 & 3474 & 77119 & 3580 & 3474 & 77119 & 3580 & 3683 & 77119 & & & 77119 & & & 77134 & & 3683 & 77119 & 3769 & 4079 & 72108 \\
\hline & 4 & 135195 & 140197 & 124180 & 135195 & 128190 & 169212 & 135195 & 128190 & 169212 & 135195 & 140197 & 169212 & 141199 & 128192 & 169212 & 157195 & 165195 & 167204 & 135195 & 140197 & 169212 & 124195 & 134186 & 165223 \\
\hline & & & & & & & & & & & & & & & & & & & & & & & & & \\
\hline & 6 & & & & & & & & & & & 106 & & & & 96 & & & & & & & & & \\
\hline & & 179217 & 179217 & 184219 & 174215 & 167208 & 174214 & 178216 & 176216 & 176216 & 175215 & 180218 & 176216 & 179217 & 183220 & 180217 & 200212 & 220 & 200234 & 179217 & 179217 & 174215 & 167218 & 181208 & 152196 \\
\hline & & & & & & & & & & & & & & & & & & & & & & & & & \\
\hline & & & & & & & & & & & & & & & & & & & & & & & & & \\
\hline & 8 & 133164 & 132162 & & & 132162 & 145 & & 132162 & 1451 & 135166 & 140168 & 142169 & 142 & 144175 & 146174 & 144 & 149 & 110153 & 134164 & 140168 & & & & 169187 \\
\hline & & 195225 & 193224 & 200228 & 194225 & 193224 & 200227 & 190223 & 194225 & 200228 & 196226 & 197225 & 197226 & 199227 & 203232 & 201229 & 179194 & 183218 & 196224 & 195225 & 197227 & 199226 & 211232 & 207236 & 221238 \\
\hline & & 22 & 1031 & 2641 & 2243 & 2447 & 2641 & & 2239 & & 2343 & 2343 & & & & 41 & 72646 & 102456 & 23. & 22 & 92447 & 2641 & 2141 & 41 & 2745 \\
\hline & & & & & & & & & & & & & & & & & & & & & & & & & \\
\hline & 10 & 104125 & 103128 & 96119 & 118140 & 116140 & 1031 & 55129 & 106129 & 118 & 118 & 115138 & 107 & 119 & 1171 & & & & & & & & & & \\
\hline & & & & & & & & & & & & & & & & & & & & & & & & & \\
\hline & & 200228 & 205231 & 195223 & 208231 & 208232 & 200227 & 202230 & 202229 & 207229 & 209233 & 208232 & 201229 & 210233 & 208232 & 223240 & 24 & 21 & 207235 & 206231 & 231 & 202229 & 174191 & 221246 & 196230 \\
\hline & & $\begin{array}{l}2240 \\
26182\end{array}$ & $\begin{array}{c}92238 \\
5878\end{array}$ & $\begin{array}{l}4058 \\
7795\end{array}$ & $\begin{array}{l}2135 \\
5271\end{array}$ & $\begin{array}{r}2239 \\
5978\end{array}$ & $\begin{array}{l}2641 \\
5676\end{array}$ & $\begin{array}{l}2137 \\
5472\end{array}$ & $\begin{array}{l}2238 \\
5573\end{array}$ & $\begin{array}{l}2641 \\
5675\end{array}$ & 2242 & 2341 & $\begin{array}{l}2641 \\
5677\end{array}$ & 2240 & 2341 & $\begin{array}{l}2641 \\
597\end{array}$ & 1435 & 527386 & 2034 & 22 & 923 & 12641 & 3343 & & 1736 \\
\hline & & & & $\begin{array}{r}7795 \\
113132\end{array}$ & & & $\begin{array}{r}5676 \\
94113\end{array}$ & & & & & & $\begin{array}{l}567 \\
891\end{array}$ & & & & & & & & & & & & \\
\hline & 12 & $\begin{array}{l}103121 \\
141\end{array}$ & $\begin{array}{r}999119 \\
140162\end{array}$ & $\begin{array}{l}11313132 \\
15169\end{array}$ & $\begin{array}{l}91110 \\
130151\end{array}$ & $\begin{array}{r}988118 \\
139159\end{array}$ & $\begin{array}{l}94113 \\
133153\end{array}$ & $\begin{array}{l}90100 \\
12914\end{array}$ & $\begin{array}{r}91110 \\
129149\end{array}$ & $\begin{array}{l}931111 \\
131151\end{array}$ & $\begin{array}{l}100119 \\
139159\end{array}$ & $\begin{array}{l}100119 \\
139159\end{array}$ & $\begin{array}{l}89108 \\
127147\end{array}$ & $\begin{array}{l}104127 \\
149\end{array}$ & $\begin{array}{l}102121 \\
140\end{array}$ & $\begin{array}{l}101124 \\
151\end{array}$ & $\begin{array}{r}8690 \\
137163\end{array}$ & $\begin{array}{l}131152 \\
164185\end{array}$ & $\begin{array}{r}7480 \\
94129\end{array}$ & $\begin{array}{l}100119 \\
139158\end{array}$ & $\begin{array}{l}105126 \\
147168\end{array}$ & $\begin{array}{l}102124 \\
147169\end{array}$ & 133149 & $\begin{array}{l}172146 \\
171193\end{array}$ & $\begin{array}{l}105113 \\
135\end{array}$ \\
\hline & & & 185208 & 184201 & 171191 & 178197 & 174194 & 170191 & 169191 & 173193 & & & 169191 & & & 1982 & 176188 & 192212 & 1441 & 178199 & 191213 & 190212 & 156176 & 203225 & 170183 \\
\hline & & 217237 & 232 & 218236 & 212234 & 216236 & 214234 & 212234 & 212234 & 213234 & 218237 & 217237 & 212234 & 224240 & 219238 & 230242 & 204222 & 222 & 214238 & 219239 & 234 & 233 & 211226 & 233246 & 200221 \\
\hline
\end{tabular}


Table 3. Cont

\begin{tabular}{|c|c|c|c|c|c|c|c|c|c|c|c|c|c|c|c|c|c|c|c|c|c|c|c|c|c|}
\hline \multirow{2}{*}{$\begin{array}{c}\text { TEST } \\
\text { IMAGES }\end{array}$} & \multirow{2}{*}{ K } & \multicolumn{3}{|c|}{ TLMVO } & \multicolumn{3}{|c|}{ LMVO } & \multicolumn{3}{|c|}{ MVO } & \multicolumn{3}{|c|}{ ALO } & \multicolumn{3}{|c|}{ DA } & \multicolumn{3}{|c|}{ FPA } & \multicolumn{3}{|c|}{ PSO } & \multicolumn{3}{|c|}{ CS } \\
\hline & & $\mathbf{R}$ & G & B & $\mathbf{R}$ & G & B & $\mathbf{R}$ & G & B & $\mathbf{R}$ & G & B & $\mathbf{R}$ & G & B & $\mathbf{R}$ & G & B & $\mathbf{R}$ & G & B & $\mathbf{R}$ & G & B \\
\hline \multirow{17}{*}{5} & 4 & $\begin{array}{c}5488 \\
139203\end{array}$ & $\begin{array}{c}4686 \\
169217\end{array}$ & $\begin{array}{c}64121 \\
189226\end{array}$ & $\begin{array}{r}4388 \\
139203\end{array}$ & $\begin{array}{r}53103 \\
173217\end{array}$ & $\begin{array}{l}62116 \\
170215\end{array}$ & $\begin{array}{c}5488 \\
139203\end{array}$ & $\begin{array}{r}4686 \\
169217\end{array}$ & $\begin{array}{c}64121 \\
189226\end{array}$ & $\begin{array}{r}4688 \\
139203\end{array}$ & $\begin{array}{r}53104 \\
175217\end{array}$ & $\begin{array}{r}64121 \\
189226\end{array}$ & $\begin{array}{r}57100 \\
171214\end{array}$ & $\begin{array}{c}4898 \\
169217\end{array}$ & $\begin{array}{r}65124 \\
189226\end{array}$ & $\begin{array}{r}54104 \\
172205\end{array}$ & $\begin{array}{r}52118 \\
175226\end{array}$ & $\begin{array}{r}54118 \\
171221\end{array}$ & $\begin{array}{c}5488 \\
139203\end{array}$ & $\begin{array}{r}53104 \\
175217\end{array}$ & $\begin{array}{c}64121 \\
189226\end{array}$ & $\begin{array}{c}4789 \\
137212\end{array}$ & $\begin{array}{r}58111 \\
165217\end{array}$ & $\begin{array}{l}84131 \\
179224\end{array}$ \\
\hline & 6 & $\begin{array}{c}3468 \\
101142\end{array}$ & $\begin{array}{r}3977 \\
117159\end{array}$ & $\begin{array}{c}3473 \\
113154\end{array}$ & $\begin{array}{c}4385 \\
118158\end{array}$ & $\begin{array}{c}3872 \\
112155\end{array}$ & $\begin{array}{c}3473 \\
113154\end{array}$ & $\begin{array}{c}3465 \\
100140\end{array}$ & $\begin{array}{c}3872 \\
112155 \\
\end{array}$ & $\begin{array}{c}3874 \\
114154\end{array}$ & $\begin{array}{c}3468 \\
101139\end{array}$ & $\begin{array}{c}3975 \\
114155\end{array}$ & $\begin{array}{c}3876 \\
116156\end{array}$ & $\begin{array}{c}3469 \\
103143 \\
\end{array}$ & $\begin{array}{c}3977 \\
117159\end{array}$ & $\begin{array}{c}3473 \\
116156\end{array}$ & $\begin{array}{c}5086 \\
105126\end{array}$ & $\begin{array}{l}285575 \\
119153\end{array}$ & $\begin{array}{c}3352 \\
71118\end{array}$ & $\begin{array}{c}3465 \\
100141\end{array}$ & $\begin{array}{c}3977 \\
117159\end{array}$ & $\begin{array}{c}3876 \\
116156\end{array}$ & $\begin{array}{r}4480 \\
128160\end{array}$ & $\begin{array}{r}5591 \\
127169\end{array}$ & $\begin{array}{r}3577 \\
109132\end{array}$ \\
\hline & & 187223 & 195221 & 194226 & 196224 & 192220 & 194226 & 187223 & 192220 & 194226 & 180214 & 192220 & 194226 & 187223 & 195221 & 194226 & 181210 & & 159210 & 187223 & 195221 & 194226 & 205235 & 199234 & 198228 \\
\hline & & 2957 & 2648 & 2456 & 3461 & 2652 & 2251 & 3458 & 2648 & 2250 & 2957 & 2652 & 3462 & 2958 & 2653 & 2456 & 2346 & 296174 & 3589 & 2958 & 2653 & 2456 & 2646 & 1835 & 3154 \\
\hline & & & & & & & & & & & & & & & & & & & & & & & & & \\
\hline & 8 & 143175 & 132165 & 147178 & 145175 & 141173 & 142173 & 144175 & 130164 & 140172 & 142175 & 145175 & 149 & 147178 & 150181 & 148178 & 1441 & 194230 & 182196 & $\begin{array}{l}\text { oo } \\
145\end{array}$ & 147178 & $\begin{array}{r}144174 \\
144\end{array}$ & $\begin{array}{r}131019 \\
1409\end{array}$ & 149 & 156187 \\
\hline & & 202227 & 195221 & 205227 & 202227 & 202226 & 203227 & 203232 & 195221 & 203226 & 202227 & 202226 & 205227 & 203228 & 206234 & $\begin{array}{l}205227 \\
205\end{array}$ & 217227 & 239 & 210234 & 202227 & 206234 & 203227 & 203231 & 185221 & 205231 \\
\hline & & $\begin{array}{l}2143 \\
6588\end{array}$ & $\begin{array}{l}2645 \\
6590\end{array}$ & $\begin{array}{l}1839 \\
6387\end{array}$ & $\begin{array}{l}2043 \\
6588\end{array}$ & $\begin{array}{l}2239 \\
6182\end{array}$ & $\begin{array}{l}1534 \\
5983\end{array}$ & $\begin{array}{l}2143 \\
6588\end{array}$ & $\begin{array}{l}2543 \\
6285\end{array}$ & $\begin{array}{l}1738 \\
688\end{array}$ & $\begin{array}{l}2143 \\
6588\end{array}$ & 2648 & 1841 & $\begin{array}{l}3057 \\
85107\end{array}$ & 2648 & $\begin{array}{l}1843 \\
7199\end{array}$ & 2952 & $\begin{array}{r}427593 \\
106911\end{array}$ & $\begin{array}{l}83261 \\
7197\end{array}$ & 2143 & 2648 & 1839 & 62664 & 4866 & 1843 \\
\hline & 10 & $\begin{array}{c}6588 \\
111138\end{array}$ & $\begin{array}{r}6590 \\
116142\end{array}$ & $\begin{array}{r}6387 \\
111135\end{array}$ & $\begin{array}{r}6588 \\
110135\end{array}$ & $\begin{array}{c}6182 \\
106134\end{array}$ & $\begin{array}{r}5983 \\
108133\end{array}$ & $\begin{array}{c}6588 \\
108130\end{array}$ & $\begin{array}{r}6385 \\
112140\end{array}$ & $\begin{array}{c}6083 \\
106130\end{array}$ & $\begin{array}{r}6588 \\
112139\end{array}$ & $\begin{array}{l}7397 \\
121146\end{array}$ & $\begin{array}{c}6590 \\
115140\end{array}$ & $\begin{array}{l}85507 \\
130155\end{array}$ & $\begin{array}{l}75102 \\
128153\end{array}$ & $\begin{array}{r}7199 \\
127154\end{array}$ & $\begin{array}{l}78112 \\
126131\end{array}$ & $\begin{array}{l}106114 \\
128145\end{array}$ & $\begin{array}{r}7197 \\
124153\end{array}$ & $\begin{array}{r}6588 \\
112138\end{array}$ & & $\begin{array}{r}6489 \\
114139\end{array}$ & $\begin{array}{r}899116 \\
142150\end{array}$ & $\begin{array}{r}8088 \\
113143\end{array}$ & $\begin{array}{r}5498 \\
113136\end{array}$ \\
\hline & & 165190 & 169195 & 160186 & 161187 & 161188 & 159186 & 153180 & 165191 & 157184 & 165190 & 167192 & 165191 & 178201 & 178198 & 176198 & 161176 & 176227 & 188216 & 165190 & & & 175204 & & 154165 \\
\hline & & 214235 & 215234 & 208231 & 209232 & 213234 & 208231 & 203230 & 213234 & 208231 & 212235 & 213234 & 213232 & 221239 & 217238 & 215235 & 204222 & 244 & 229 & 214235 & 217234 & 210231 & 224 & 206230 & 187213 \\
\hline & & 1834 & 2543 & 1122 & 1429 & 2542 & 1734 & 1936 & 2238 & 1128 & 2142 & 2543 & 1734 & 2847 & 2643 & 1738 & 1447 & 314763 & 1633 & 2143 & 2539 & 1434 & 3649 & 2131 & 4252 \\
\hline & & & & & & & & & & & & & & & & & & & & & & & & & \\
\hline & 12 & 88109 & 106129 & 7292 & 7793 & 102123 & 941 & & 94120 & 86105 & 99119 & & & 103121 & & & & & & & & & & & 117137 \\
\hline & 12 & 132155 & 152175 & 1141 & 112133 & 143163 & 135 & 129151 & 139158 & 127148 & 140161 & & & & & & & & & & & & & & \\
\hline & & & 195213 & & & & & & & 171194 & & & & & & & & & & & & & & & \\
\hline & & 223239 & 226242 & 208231 & 203232 & 219238 & 215235 & 214235 & 215234 & 213231 & 223240 & 217234 & 213232 & 216235 & 217238 & 218235 & 224246 & 247 & 229238 & 223240 & 217234 & 215235 & 229241 & 219246 & 207234 \\
\hline & 4 & $\begin{array}{l}2575 \\
\end{array}$ & 4581 & $\begin{array}{r}3884 \\
\end{array}$ & $\begin{array}{r}2575 \\
\end{array}$ & 4581 & 3884 & $\begin{array}{l}2575 \\
277175\end{array}$ & $\begin{array}{r}4581 \\
\end{array}$ & $\begin{array}{r}3884 \\
\end{array}$ & $\begin{array}{r}2575 \\
\end{array}$ & 4581 & 3884 & $\begin{array}{r}2575 \\
127175\end{array}$ & 4581 & $\begin{array}{r}3834 \\
8917\end{array}$ & $\begin{array}{c}3795 \\
137173\end{array}$ & & $\begin{array}{r}2782 \\
11173\end{array}$ & $\begin{array}{l}2575 \\
127175\end{array}$ & 4581 & 3884 & 2481 & 4772 & $\begin{array}{r}4284 \\
\end{array}$ \\
\hline & 4 & 127175 & 128170 & 129172 & 127175 & 128170 & 129172 & 127175 & 129172 & 129172 & 127175 & 129172 & 129172 & 127175 & 130175 & 129172 & 137173 & 122158 & 118173 & 127175 & 129172 & 129172 & 123169 & 132173 & 128174 \\
\hline & & & 4268 & 2756 & 1653 & 4063 & 2251 & 1652 & 4268 & 2756 & 1654 & 4268 & 2756 & 2059 & 4270 & 2756 & 65095 & 507288 & 1653 & 1654 & 4268 & 2656 & & & \\
\hline & 6 & $\begin{array}{c}9012 \\
163197\end{array}$ & $\begin{array}{r}98129 \\
159192\end{array}$ & $\begin{array}{c}87118 \\
149182\end{array}$ & $\begin{array}{c}89127 \\
163197\end{array}$ & $\begin{array}{c}93127 \\
159192\end{array}$ & $\begin{array}{c}83116 \\
149182\end{array}$ & $\begin{array}{c}88126 \\
163197\end{array}$ & $\begin{array}{r}97128 \\
158192\end{array}$ & $\begin{array}{c}87118 \\
149182\end{array}$ & $\begin{array}{c}91128 \\
163197\end{array}$ & $\begin{array}{c}98129 \\
159192\end{array}$ & $\begin{array}{c}87118 \\
149182\end{array}$ & $\begin{array}{r}96133 \\
168199\end{array}$ & $\begin{array}{r}99129 \\
159192\end{array}$ & $\begin{array}{r}87119 \\
151183\end{array}$ & $\begin{array}{c}124177 \\
218\end{array}$ & $\begin{array}{c}108144 \\
169\end{array}$ & $\begin{array}{r}7396 \\
119155\end{array}$ & $\begin{array}{r}91128 \\
163197\end{array}$ & $\begin{array}{r}98129 \\
159192\end{array}$ & $\begin{array}{c}87118 \\
149182\end{array}$ & $\begin{array}{c}6291 \\
131190\end{array}$ & & $\begin{array}{c}82104 \\
133171\end{array}$ \\
\hline & & 1541 & & & & & & & & & & & & & 4059 & & & & 2658 & & & & & & \\
\hline & & & & & & & & & & & & & & & & & & & & & & & & & \\
\hline & 8 & 121147 & 126149 & 110136 & 136168 & 119143 & & 119146 & 124147 & 114 & 122148 & 124146 & 140165 & 125152 & 126148 & 117141 & 137189 & 111122 & 162191 & 122148 & & 118143 & & & 100117 \\
\hline & & 174202 & 172195 & 162189 & 197226 & 167193 & 168193 & 173202 & 170194 & 165190 & 175202 & 169193 & 190 & 177204 & 171194 & 167193 & 200224 & 171 & 206221 & 175202 & 172195 & 168193 & 186211 & 184204 & 130183 \\
\hline & & 1027 & & & 1232 & 3245 & & & & & & 34 & 1331 & & & & & 203750 & & & & & & & \\
\hline & & & & & & & & & & & & & & & & & & & & & & & & & \\
\hline & 10 & 851 & 109128 & & & & & & & & & & & & & & & & & & & & & & \\
\hline & & 135162 & & & & & & & & & & & & & & & & & & & & & & & \\
\hline & & 187212 & 188207 & 184219 & 197226 & 189218 & 186219 & 187210 & 176196 & 175197 & 191214 & 195219 & 177198 & 212231 & 196219 & 179201 & 217232 & 210 & 195214 & 190212 & 188207 & 176198 & 195208 & 164182 & 169194 \\
\hline & & & & 717 & & & & & & & & & & & & & & & & & & & & & \\
\hline & & & & & & & & & & & & & & & & & & & & & & & & & \\
\hline & & & & $\begin{array}{l}750 \\
7592\end{array}$ & 90110 & 96114 & 87106 & $\begin{array}{l}40.7 \\
81101\end{array}$ & 91108 & 82102 & & 95112 & 89 & 90110 & 97118 & 89109 & & 117126 & 91121 & 89109 & & 86 & & 7378 & 88123 \\
\hline & 12 & 128148 & 122138 & 111131 & 129148 & 133152 & 125144 & 119138 & 124140 & 123142 & 131150 & 129146 & 128 & 129147 & 138158 & 130151 & 107128 & 166175 & $\begin{array}{l}139162 \\
1392\end{array}$ & 128148 & 131149 & 125144 & 88113 & 92101 & 27141 \\
\hline & & & 154171 & 150172 & 168187 & 173194 & 1621 & 159180 & 158177 & 160180 & 169187 & 163181 & 163 & 165183 & 174191 & 168 & 163190 & 186202 & 178183 & 168187 & 168186 & 162181 & 143164 & 137165 & 164189 \\
\hline & & 207231 & 189207 & 195 & 206226 & 218244 & 199219 & 202226 & 196219 & 199219 & 206226 & 199219 & 198219 & 204226 & 206219 & 202219 & 210239 & 226 & 184196 & 206226 & 203219 & 201219 & 196221 & 186207 & 211225 \\
\hline
\end{tabular}


Table 3. Cont

\begin{tabular}{|c|c|c|c|c|c|c|c|c|c|c|c|c|c|c|c|c|c|c|c|c|c|c|c|c|c|}
\hline \multirow{2}{*}{$\begin{array}{c}\text { TEST } \\
\text { IMAGES }\end{array}$} & \multirow{2}{*}{ K } & \multicolumn{3}{|c|}{ TLMVO } & \multicolumn{3}{|c|}{ LMVO } & \multicolumn{3}{|c|}{ MVO } & \multicolumn{3}{|c|}{ ALO } & \multicolumn{3}{|c|}{ DA } & \multicolumn{3}{|c|}{ FPA } & \multicolumn{3}{|c|}{ PSO } & \multicolumn{3}{|c|}{ CS } \\
\hline & & $\mathbf{R}$ & G & B & $\mathbf{R}$ & G & B & $\mathbf{R}$ & G & B & $\mathbf{R}$ & G & B & $\mathbf{R}$ & G & B & $\mathbf{R}$ & G & B & $\mathrm{R}$ & G & B & $\mathbf{R}$ & G & B \\
\hline \multirow{19}{*}{7} & 4 & $\begin{array}{r}64103 \\
151225\end{array}$ & $\begin{array}{r}5690 \\
158199\end{array}$ & $\begin{array}{r}54127 \\
158185\end{array}$ & $\begin{array}{r}64106 \\
153221\end{array}$ & $\begin{array}{r}5490 \\
158199\end{array}$ & $\begin{array}{c}3772 \\
138180\end{array}$ & $\begin{array}{r}64106 \\
153221\end{array}$ & $\begin{array}{r}5690 \\
158199\end{array}$ & $\begin{array}{c}3772 \\
138180\end{array}$ & $\begin{array}{r}64106 \\
153221\end{array}$ & $\begin{array}{c}5690 \\
158199\end{array}$ & $\begin{array}{c}3772 \\
138180\end{array}$ & $\begin{array}{r}64106 \\
153225\end{array}$ & $\begin{array}{c}5692 \\
167207\end{array}$ & $\begin{array}{r}3772 \\
138180\end{array}$ & $\begin{array}{l}80101 \\
151229\end{array}$ & $\begin{array}{c}70132 \\
177199\end{array}$ & $\begin{array}{c}5290 \\
126162\end{array}$ & $\begin{array}{r}64103 \\
151225\end{array}$ & $\begin{array}{c}5690 \\
158199\end{array}$ & $\begin{array}{r}3772 \\
138180\end{array}$ & $\begin{array}{c}73113 \\
159226\end{array}$ & $\begin{array}{c}6399 \\
151189\end{array}$ & $\begin{array}{r}4368 \\
135177\end{array}$ \\
\hline & & 5891 & 5483 & 3665 & 4474 & 4573 & 3454 & 5888 & 4373 & 3662 & 5892 & 5483 & 3661 & 5892 & 5484 & 3460 & 5873 & 305597 & 2560 & 5891 & 4573 & 3665 & 5387 & 4376 & 3267 \\
\hline & 6 & 126161 & & 103134 & 115153 & & & 121154 & & 93130 & 126161 & & 83127 & 129162 & 115149 & 83127 & 99130 & & 109134 & 126161 & 102144 & 99130 & 115151 & 96131 & 111146 \\
\hline & & 201232 & 179207 & 160185 & 194229 & 178207 & 152185 & 193228 & 178207 & 158185 & 201232 & 179207 & 158185 & 201232 & 179207 & 158185 & 179214 & 193 & 148179 & 201232 & 178207 & 158185 & 180219 & 162203 & 167189 \\
\hline & & 4468 & 4065 & 2350 & 4464 & 5274 & 2143 & 4468 & 4063 & 1837 & 4468 & 4063 & 3454 & 4474 & 4061 & 3454 & 3144 & 406178 & 4561 & 4468 & 4063 & 2142 & 4268 & 4554 & 1436 \\
\hline & 8 & & & & & & & & & & & & & & & & & & & & & & & & \\
\hline & & 147173 & 149176 & 121142 & 144171 & 154178 & 126152 & 147173 & 142171 & 110136 & 150175 & 144172 & 120142 & 157186 & 141171 & 134158 & 1391 & 182196 & 139165 & 147173 & $14 \mathrm{I} / 2$ & 136 & 128155 & 48 & \\
\hline & & 205233 & 198219 & 163185 & 204233 & 198219 & 170190 & 205233 & 195219 & 160185 & 205233 & 198219 & 163185 & 212234 & 196219 & 179199 & 192243 & 211 & 183191 & 205233 & 198219 & 160185 & 176221 & 175207 & 170187 \\
\hline & & 4464 & 3454 & 1834 & 4158 & 3452 & & 4464 & 3956 & 1836 & 4468 & 3454 & 2137 & 4464 & 4061 & 3454 & 3569 & 245273 & 2940 & 4464 & 4059 & 2137 & 5471 & 3365 & 2862 \\
\hline & & & & & & & & & & & & & & & & & & & & & & & & & \\
\hline & 10 & 129150 & 110133 & 85110 & 121144 & 107132 & 107130 & 124146 & 112136 & 85106 & 136155 & 112134 & & 133155 & 132156 & 110127 & 129145 & 105157 & & 129151 & 118139 & & 120136 & 135143 & 118140 \\
\hline & & 171194 & 156177 & 133156 & 166190 & 156178 & 152170 & 169191 & 157176 & 127147 & 175197 & 158179 & 138156 & 178201 & 178195 & 143160 & 166184 & 187199 & 157162 & 173196 & 158179 & 143163 & 152158 & 166185 & 155166 \\
\hline & & 217235 & 198219 & 177196 & 214234 & 198219 & 185201 & 214234 & 198219 & 164185 & 217236 & 199219 & 172190 & 221238 & 208225 & 180198 & 193229 & 226 & 181192 & 218238 & 199219 & 185256 & 170227 & 197219 & 201218 \\
\hline & & 3244 & 3452 & 1833 & 3244 & 2942 & 1834 & 4158 & 3140 & 2136 & 4462 & 3452 & 2137 & 4464 & 3451 & 2136 & 3765 & 435896 & 1831 & 4464 & 3455 & 1836 & 2452 & 2672 & 1326 \\
\hline & & & & & & & & & & & & & & & & & & & & & & & & & \\
\hline & 12 & & & & & & & & & & & & & & & & & & & & & & & & \\
\hline & & & & 110129 & & & & & & & & & & & & & & & & & & & & & \\
\hline & & & & & & & & & & & & & & & & & & & & & & & & & \\
\hline & & 214234 & 207225 & 185200 & 211233 & 199219 & 185201 & 214234 & 199219 & 179196 & 221237 & 208225 & 179197 & 228240 & 207225 & 180201 & 210236 & 237 & 186218 & 221237 & 208225 & 185201 & 207218 & 210228 & 170191 \\
\hline & & & & & & & & & & & & & & & & & & & & & & & & & \\
\hline & 4 & 126164 & 151223 & 146195 & 126164 & 157223 & 146195 & 126164 & 157223 & 181230 & 126164 & 15723 & 146195 & 126164 & 157223 & 181230 & 107157 & 173232 & 185232 & 126164 & 157223 & 181230 & 118164 & 152224 & 187231 \\
\hline & & & & & & & & & & & & & & & & & & & & & & & & & \\
\hline & 6 & & & & & & & & & & & & & & & & & & & & & & & & \\
\hline & & 150170 & 204225 & 196230 & 150170 & 207225 & 195230 & 150170 & 174223 & 195230 & 135164 & 176223 & 196230 & 135164 & 207225 & 196230 & 163182 & 226239 & 193239 & 150170 & 176223 & 196230 & 148171 & 204229 & 197228 \\
\hline & & & & & & & & & & & & & & & & & & & & & & & & & \\
\hline & & & & & & & & & & & & & & & & & & & & & & & & & \\
\hline & 8 & 109132 & 145176 & 137167 & 109132 & 133166 & 144 & 106 & 146177 & 144 & 109 & 148178 & 146 & 106 & 147178 & 165 & 38151 & 200215 & & & 148 & & 110 & 129 & 151183 \\
\hline & & 153170 & 207225 & 199230 & 153170 & 207225 & 202230 & 153170 & 207225 & 202230 & 153170 & 207225 & 203231 & 153170 & 207225 & 225241 & 164195 & 237 & 235245 & 153170 & 207225 & 202230 & 158168 & 213235 & 214233 \\
\hline & & 1534 & 2242 & 2446 & 1635 & 2039 & 2345 & 1432 & 2447 & & 1327 & 2447 & 2853 & & 2558 & 2446 & 51637 & 2572 & 2027 & & 2449 & 2446 & & 2540 & 1956 \\
\hline & & & & & & & & & & & & & & & & & & & & & & & & & \\
\hline & 10 & 94114 & 107129 & 1181 & 95115 & 104129 & 1181 & & 125153 & 112134 & & 116140 & 128 & & 140167 & 121 & & & & & & & & & \\
\hline & & & & & & & & & & & & & & & & & 127 & & & & & & & & \\
\hline & & 170187 & 207225 & 225241 & 170182 & 207225 & 224241 & 164181 & 223239 & 204230 & 153170 & 207225 & 225241 & 153170 & 223239 & 225241 & 1 & 231 & 234241 & 170256 & 223239 & 205231 & 160175 & 207214 & 205223 \\
\hline & & 1329 & 2444 & 2140 & 1329 & $\begin{array}{l}2037 \\
547\end{array}$ & 1937 & 1328 & $\begin{array}{l}2039 \\
5897\end{array}$ & $\begin{array}{l}1837 \\
576\end{array}$ & 1328 & 2447 & 23 & $\begin{array}{l}1634 \\
5257\end{array}$ & 224 & 25 & 123 & 725 & & 13 & & & 16 & & 3448 \\
\hline & & & & & & & & & $\begin{array}{r}5879 \\
100121\end{array}$ & $\begin{array}{l}5776 \\
96115\end{array}$ & $\begin{array}{l}4358 \\
7489\end{array}$ & & & & & & $\begin{array}{l}4249 \\
7585\end{array}$ & & & & & & & & 6494 \\
\hline & 12 & $\begin{array}{r}7793 \\
109124\end{array}$ & $\begin{array}{l}106126 \\
1466\end{array}$ & $\begin{array}{l}100120 \\
140161\end{array}$ & $\begin{array}{l}11100 \\
118135\end{array}$ & $\begin{array}{l}941144 \\
135158\end{array}$ & $\begin{array}{l}95116 \\
137158\end{array}$ & $\begin{array}{r}7792 \\
108123\end{array}$ & $\begin{array}{l}1001121 \\
142164\end{array}$ & $\begin{array}{l}9615 \\
136157\end{array}$ & $\begin{array}{r}7489 \\
104121\end{array}$ & $\begin{array}{l}105124 \\
143163\end{array}$ & $\begin{array}{l}115125 \\
143163\end{array}$ & $\begin{array}{r}901078 \\
123138\end{array}$ & $\begin{array}{l}108129 \\
14969\end{array}$ & $\begin{array}{l}106126 \\
1489\end{array}$ & $\begin{array}{r}7585 \\
103110\end{array}$ & $\begin{array}{l}1541180 \\
175\end{array}$ & $\begin{array}{r}9096 \\
12130\end{array}$ & $\begin{array}{r}7590 \\
105121\end{array}$ & $\begin{array}{l}106127 \\
1468\end{array}$ & $\begin{array}{l}102122 \\
143164\end{array}$ & $\begin{array}{l}6861 \\
8998\end{array}$ & $\begin{array}{l}109141 \\
156176\end{array}$ & $\begin{array}{l}113122 \\
126163\end{array}$ \\
\hline & & & 186207 & 182204 & 153170 & 181204 & 1802 & & 18520 & 180203 & 138 & 1872 & 1852 & 153 & 188207 & 1892 & 121 & 200226 & 137 & 138 & 188 & 185 & 115129 & 193209 & \\
\hline & & 170187 & 223239 & 225241 & 187236 & 222239 & 224241 & 170239 & 222239 & 225241 & 170189 & 223239 & 225241 & 187256 & 223239 & 225241 & 136186 & 236 & 200232 & 170187 & 223239 & 225241 & 144166 & 222241 & 211236 \\
\hline
\end{tabular}


Table 3. Cont.

\begin{tabular}{|c|c|c|c|c|c|c|c|c|c|c|c|c|c|c|c|c|c|c|c|c|c|c|c|c|c|}
\hline \multirow{2}{*}{$\begin{array}{c}\text { TEST } \\
\text { IMAGES }\end{array}$} & \multirow{2}{*}{ K } & \multicolumn{3}{|c|}{ TLMVO } & \multicolumn{3}{|c|}{ LMVO } & \multicolumn{3}{|c|}{ MVO } & \multicolumn{3}{|c|}{ ALO } & \multicolumn{3}{|c|}{ DA } & \multicolumn{3}{|c|}{ FPA } & \multicolumn{3}{|c|}{ PSO } & \multicolumn{3}{|c|}{ CS } \\
\hline & & $\mathbf{R}$ & G & B & $\mathbf{R}$ & G & B & $\mathbf{R}$ & G & B & $\mathbf{R}$ & G & B & $\mathbf{R}$ & G & B & $\mathbf{R}$ & G & B & $\mathbf{R}$ & G & B & $\mathbf{R}$ & G & B \\
\hline \multirow{16}{*}{9} & 4 & $\begin{array}{r}62111 \\
164212 \\
\end{array}$ & $\begin{array}{c}2786 \\
152218 \\
\end{array}$ & $\begin{array}{c}4488 \\
120154 \\
\end{array}$ & $\begin{array}{r}62111 \\
164212 \\
\end{array}$ & $\begin{array}{r}2786 \\
154222 \\
\end{array}$ & $\begin{array}{c}4485 \\
116154 \\
\end{array}$ & $\begin{array}{r}62111 \\
163212 \\
\end{array}$ & $\begin{array}{c}2786 \\
154222 \\
\end{array}$ & $\begin{array}{c}4888 \\
120154 \\
\end{array}$ & $\begin{array}{r}62111 \\
164212 \\
\end{array}$ & $\begin{array}{c}2786 \\
154222 \\
\end{array}$ & $\begin{array}{c}4888 \\
120154 \\
\end{array}$ & $\begin{array}{r}62111 \\
164212 \\
\end{array}$ & $\begin{array}{r}2786 \\
154222 \\
\end{array}$ & $\begin{array}{c}4888 \\
120154 \\
\end{array}$ & $\begin{array}{r}65119 \\
154213 \\
\end{array}$ & $\begin{array}{c}3172 \\
154203 \\
\end{array}$ & $\begin{array}{c}5498 \\
137180 \\
\end{array}$ & $\begin{array}{r}62111 \\
164212 \\
\end{array}$ & $\begin{array}{r}2786 \\
154222 \\
\end{array}$ & $\begin{array}{c}4485 \\
120154 \\
\end{array}$ & $\begin{array}{l}84123 \\
170216 \\
\end{array}$ & $\begin{array}{r}2770 \\
125165 \\
\end{array}$ & $\begin{array}{r}4886 \\
120162 \\
\end{array}$ \\
\hline & 6 & $\begin{array}{c}3070 \\
107146\end{array}$ & $\begin{array}{c}2768 \\
106145\end{array}$ & $\begin{array}{r}3057 \\
85109\end{array}$ & $\begin{array}{r}1953 \\
91131\end{array}$ & $\begin{array}{c}2768 \\
106147\end{array}$ & $\begin{array}{r}3057 \\
85109\end{array}$ & $\begin{array}{c}3070 \\
107146\end{array}$ & $\begin{array}{c}2765 \\
100139\end{array}$ & & $\begin{array}{c}1961 \\
99139\end{array}$ & $\begin{array}{c}2768 \\
106145\end{array}$ & $\begin{array}{r}3057 \\
85109\end{array}$ & $\begin{array}{c}3173 \\
111149\end{array}$ & $\begin{array}{c}2765 \\
101144\end{array}$ & $\begin{array}{r}3159 \\
85109\end{array}$ & $\begin{array}{c}5083 \\
142154\end{array}$ & $\begin{array}{r}32114 \\
127160\end{array}$ & $\begin{array}{r}1825 \\
5594\end{array}$ & $\begin{array}{c}3473 \\
111149\end{array}$ & $\begin{array}{c}2769 \\
109149\end{array}$ & $\begin{array}{r}3057 \\
85109\end{array}$ & $\begin{array}{c}3568 \\
110165\end{array}$ & $\begin{array}{r}3048 \\
79112\end{array}$ & $\begin{array}{r}2570 \\
92115\end{array}$ \\
\hline & & 182219 & 185222 & 132158 & 173219 & 188227 & 135167 & 182219 & 181222 & 132158 & 179219 & 185222 & 132158 & 186219 & 188227 & 132161 & 178227 & 190217 & 130184 & 186219 & 189227 & 135167 & 196217 & 159198 & 143162 \\
\hline & 8 & $\begin{array}{c}1847 \\
77107 \\
138168\end{array}$ & $\begin{array}{r}2758 \\
85112 \\
139169\end{array}$ & $\begin{array}{r}2748 \\
6889 \\
109127\end{array}$ & $\begin{array}{c}1844 \\
73101 \\
129159\end{array}$ & $\begin{array}{r}2141 \\
6593 \\
124156\end{array}$ & $\begin{array}{r}2548 \\
6991 \\
111132\end{array}$ & $\begin{array}{r}1845 \\
75106 \\
136166\end{array}$ & $\begin{array}{c}2257 \\
86115 \\
143172\end{array}$ & $\begin{array}{r}2545 \\
6788 \\
109127\end{array}$ & $\begin{array}{r}1847 \\
77107 \\
137167\end{array}$ & $\begin{array}{r}2257 \\
86115 \\
145175\end{array}$ & $\begin{array}{r}2748 \\
6989 \\
110132\end{array}$ & $\begin{array}{r}1850 \\
82113 \\
144172\end{array}$ & $\begin{array}{r}2258 \\
87116 \\
146176\end{array}$ & $\begin{array}{r}2952 \\
7391 \\
109127\end{array}$ & $\begin{array}{c}5388 \\
123152 \\
171203\end{array}$ & $\begin{array}{c}2577 \\
110147 \\
173205\end{array}$ & $\begin{array}{r}5269 \\
7795 \\
103129\end{array}$ & $\begin{array}{r}1847 \\
77106 \\
136166\end{array}$ & $\begin{array}{c}2258 \\
86115 \\
145175\end{array}$ & $\begin{array}{r}2748 \\
7091 \\
109132\end{array}$ & $\begin{array}{c}3558 \\
91105 \\
124150\end{array}$ & $\begin{array}{r}2960 \\
88122 \\
157177\end{array}$ & $\begin{array}{c}4267 \\
81100 \\
122140\end{array}$ \\
\hline & & $\begin{array}{l}1907222 \\
197\end{array}$ & 199227 & 153178 & 188219 & 191227 & 154178 & $\begin{array}{l}194222 \\
194\end{array}$ & $\begin{array}{l}204231 \\
204\end{array}$ & 152178 & 197222 & 206234 & $\begin{array}{l}110132 \\
154178 \\
\end{array}$ & $\begin{array}{l}144172 \\
199222\end{array}$ & $\begin{array}{l}146176 \\
206234\end{array}$ & $\begin{array}{l}109127 \\
149172\end{array}$ & $\begin{array}{l}2712203 \\
22244\end{array}$ & $\begin{array}{l}227237 \\
2737\end{array}$ & $\begin{array}{l}10349 \\
154171\end{array}$ & $\begin{array}{l}136106 \\
197222\end{array}$ & $\begin{array}{l}154175 \\
206234\end{array}$ & $\begin{array}{l}109132 \\
15478\end{array}$ & $\begin{array}{l}124150 \\
190219\end{array}$ & 185210 & $\begin{array}{l}122140 \\
158180\end{array}$ \\
\hline & & 1835 & 2138 & 2542 & 1839 & 2136 & 2037 & 1837 & 2139 & 2441 & 1840 & 2140 & 2542 & 1843 & 2251 & 2440 & 5781 & 315594 & 1629 & 1841 & 2141 & 2440 & 3163 & 2453 & 4158 \\
\hline & & 5372 & 608 & 5977 & 6184 & 5677 & 5471 & 5677 & 6386 & 5873 & 6387 & 6588 & 5975 & 6689 & 76101 & 5673 & 99127 & 118145 & 5565 & 670 & 6590 & 5977 & 8594 & 72108 & 76104 \\
\hline & 10 & 92114 & 112137 & 93109 & 107131 & 100126 & 91109 & 96119 & 109133 & 91109 & 110132 & & 91109 & 113138 & 128152 & 91109 & & & & & & & & & \\
\hline & & 137162 & 163189 & 126143 & 154177 & 153181 & 126143 & 142167 & 159185 & 126153 & 155179 & 163187 & 125140 & 164193 & 176198 & 126143 & 189194 & 193208 & 123145 & 169194 & 168194 & 126143 & 163184 & 164178 & 149161 \\
\hline & & 190219 & 214236 & 161178 & 201224 & 209234 & 161178 & 193219 & 210234 & 178225 & 202224 & 210234 & 158178 & 219243 & 219238 & 161178 & 213237 & 230 & 166195 & 219243 & 218238 & 161178 & 210224 & 208240 & 173179 \\
\hline & & 1835 & 2135 & 2037 & 1835 & 92138 & 1932 & 1833 & 2136 & 1425 & 1840 & 2137 & 2035 & 1840 & 2141 & 1630 & 2063 & 2577 & 1219 & 1840 & 2137 & 1629 & 3874 & 3144 & 3262 \\
\hline & & & & & & & & & & & & & & & & & & & & & & & & & \\
\hline & 12 & 95115 & 90109 & 8911 & & 102124 & & & & & & & & & & & & & & & & & & & \\
\hline & 12 & 135155 & 128148 & 1201 & 6157 & & 107120 & & 1341 & & & & & & & & & & & & & & & & \\
\hline & & & & & & & & & & & & & & & & & & & & & & & & & \\
\hline & & 219240 & 214236 & 169182 & 219243 & 236 & 166182 & 212230 & 218236 & 161178 & 219243 & 222238 & 178244 & 222243 & 226242 & 169182 & 208234 & 240249 & 175209 & 224243 & 222238 & 162178 & 224230 & 212238 & 175185 \\
\hline & 4 & $\begin{array}{c}1155 \\
155202\end{array}$ & $\begin{array}{c}2792 \\
158215\end{array}$ & $\begin{array}{c}5296 \\
147196\end{array}$ & $\begin{array}{c}1154 \\
143202\end{array}$ & $\begin{array}{c}2792 \\
158215\end{array}$ & $\begin{array}{c}5296 \\
147196\end{array}$ & $\begin{array}{c}1155 \\
155202\end{array}$ & $\begin{array}{c}2792 \\
158215\end{array}$ & $\begin{array}{c}5296 \\
147196\end{array}$ & $\begin{array}{r}38100 \\
155202\end{array}$ & $\begin{array}{r}52100 \\
158215\end{array}$ & $\begin{array}{c}5296 \\
147196\end{array}$ & $\begin{array}{c}1154 \\
143202\end{array}$ & $\begin{array}{c}2792 \\
158215\end{array}$ & $\begin{array}{c}5296 \\
147196\end{array}$ & $\begin{array}{r}4386 \\
135197\end{array}$ & $\begin{array}{r}57111 \\
160206\end{array}$ & $\begin{array}{c}3997 \\
170199\end{array}$ & $\begin{array}{r}1155 \\
155202\end{array}$ & $\begin{array}{c}2792 \\
158215\end{array}$ & $\begin{array}{c}5296 \\
147196\end{array}$ & $\begin{array}{r}44105 \\
161200\end{array}$ & $\begin{array}{r}3990 \\
145220\end{array}$ & $\begin{array}{l}5111 \\
54192\end{array}$ \\
\hline & & 155202 & $\frac{158215}{2454}$ & $\begin{array}{c}147196 \\
2253\end{array}$ & $\frac{143202}{94388}$ & $\frac{158215}{2667}$ & 147196 & & & $147 / 196$ & & & & & & & & & 170199 & 155202 & & $\begin{array}{r}147196 \\
2253\end{array}$ & 161200 & 145220 & 44192 \\
\hline & 6 & $\begin{array}{l}131168 \\
131\end{array}$ & & 881 & & & & 168 & & & & & & & & & & $\begin{array}{l}15 \\
11\end{array}$ & & & & 29 & $\begin{array}{l}4196 \\
41176\end{array}$ & & \\
\hline & & 208 & 168215 & 161199 & 207 & 180217 & 182212 & 208 & 179217 & 168202 & 168208 & 171217 & 166202 & 169212 & 180217 & 168202 & 192244 & 213 & 174212 & 168208 & 180217 & 168202 & 214 & 199225 & 185213 \\
\hline & & & & & & & & & & & & & & & & & & & & & & & & & \\
\hline & & & & & & & & & & & & & & & & & & & & & & & & & \\
\hline & 8 & 166196 & 132160 & & 143172 & 137165 & & & & & 162193 & & 142170 & 162194 & 153184 & 155186 & & & & 162194 & & & & & 168196 \\
\hline & & 224 & 189220 & 188216 & 212 & 192220 & 193221 & 219 & 187220 & 197223 & 221 & 190220 & 199224 & 224 & 214234 & 214244 & 208226 & 238249 & 177199 & 224 & 189220 & 197223 & 182221 & 207225 & 215227 \\
\hline & & 93963 & 2036 & 1839 & 2950 & & & 3858 & 2349 & & 39 & 2450 & & 9406 & 2346 & 1846 & 92043 & 314749 & & 93861 & 2450 & 2249 & 43249 & 2341 & 1863 \\
\hline & & & & & & & & & & & & & & & & & & & & & & & & & \\
\hline & 10 & 141166 & 95118 & 105128 & 121145 & 103124 & 108129 & 131155 & 120144 & 110 & 32155 & 120144 & & & 115139 & & & & & 55 & & & & & 158 \\
\hline & & 189211 & 141165 & & 169 & & & 177202 & & & & & & & & & & & & & & & & & \\
\hline & & 232 & 190220 & 202226 & 222 & 196221 & 199224 & 224 & 214233 & 202225 & 228 & 214234 & 202226 & 23 & 214234 & 226244 & 25 & 22 & 205223 & 228 & 214234 & 223244 & 221 & 212227 & 229244 \\
\hline & & $\begin{array}{r}72747 \\
6990\end{array}$ & $\begin{array}{l}2342 \\
6078\end{array}$ & $\begin{array}{l}1734 \\
5270\end{array}$ & $\begin{array}{c}72341 \\
5876\end{array}$ & $\begin{array}{l}2342 \\
6079\end{array}$ & $\begin{array}{l}1838 \\
5880\end{array}$ & $\begin{array}{c}72441 \\
6081\end{array}$ & $\begin{array}{l}2036 \\
5377\end{array}$ & $\begin{array}{l}1632 \\
49966\end{array}$ & $\begin{array}{r}82849 \\
7190\end{array}$ & $\begin{array}{l}2239 \\
5676\end{array}$ & $\begin{array}{l}1836 \\
5373\end{array}$ & $\begin{array}{c}72345 \\
6590\end{array}$ & $\begin{array}{l}2448 \\
6991\end{array}$ & $\begin{array}{l}1944 \\
6587\end{array}$ & $\begin{array}{r}1747 \\
79101\end{array}$ & $\begin{array}{c}213758 \\
7997\end{array}$ & $\begin{array}{l}2649 \\
6488\end{array}$ & $\begin{array}{r}82849 \\
6990\end{array}$ & $\begin{array}{l}2138 \\
5473\end{array}$ & $\begin{array}{l}1838 \\
5880\end{array}$ & $\begin{array}{l}2155 \\
93105\end{array}$ & $\begin{array}{l}2331 \\
5261\end{array}$ & $\begin{array}{l}2327 \\
4259\end{array}$ \\
\hline & & & & & & & & & & & & & $\begin{array}{r}5373 \\
91110\end{array}$ & $\begin{array}{r}6590 \\
113131\end{array}$ & & & & 119133 & & & $\begin{array}{l}4.73 \\
1114\end{array}$ & & $\begin{array}{l}93105 \\
120130\end{array}$ & $\begin{array}{l}261 \\
2109 \\
2.09\end{array}$ & $\begin{array}{l}4559 \\
7390\end{array}$ \\
\hline & 12 & & 1371 & 1251 & 134 & 1371 & 1401 & 138 & 127147 & 123 & & 136156 & 129 & & 150170 & 149 & 152173 & 155 & & 155 & 134153 & & 142169 & 133146 & 120144 \\
\hline & & 192212 & 175194 & 1621 & 176202 & 175194 & 182202 & 177202 & 168190 & 165185 & 196216 & 175194 & 169188 & 196215 & & 1912 & & 192222 & & 196216 & 172193 & 184204 & 186202 & 175194 & 162181 \\
\hline & & 232 & 214233 & 202226 & 225 & 214234 & 223244 & 228 & 213232 & 204227 & 238 & 214233 & 207228 & 238 & 223239 & 228244 & 233253 & 232 & 196238 & 238 & 214234 & 226244 & 209237 & 205232 & 202224 \\
\hline
\end{tabular}




\subsection{Performance Metric}

We performed the experimental from both Performance evaluation and Statistical evaluation. The methods used are shown in Table 4 .

Table 4. The definition and description of performance measures.

\begin{tabular}{|c|c|c|c|c|}
\hline Category & Name & Formulation & Remark & Reference \\
\hline \multirow{6}{*}{$\begin{array}{l}\text { Performance } \\
\text { evaluation }\end{array}$} & $\begin{array}{l}\text { Structural } \\
\text { Similarity Index }\end{array}$ & $\operatorname{SSIM}(I, \hat{I})=\frac{\left(2 \mu_{1} \mu_{\hat{T}}+c_{1}\right)\left(2 \sigma_{I} \sigma_{\hat{I}}+\mathcal{c}_{2}\right)}{\left(\mu_{I}^{2}+\mu_{I}^{2}+c_{1}\right)\left(\sigma_{I}^{2}+\sigma_{I}^{2}+c_{2}\right)}$ & $\begin{array}{l}\text { The index that measures the similarity between the two images before and } \\
\text { after the segmentation, the closer the value is to } 1 \text {, the better the image } \\
\text { segmentation effect. }\end{array}$ & [64] \\
\hline & $\begin{array}{l}\text { Feature Similarity } \\
\quad \text { Index }\end{array}$ & $F S I M=\frac{\sum_{x \hat{\Omega} \Omega} S_{L}(x) \times P C_{m}(x)}{\sum_{x \hat{\Omega} \Omega} P C_{m}(x)}$ & $\begin{array}{l}\text { An indicator for evaluating the local structural importance between the } \\
\text { original image and the segmented image, the maximum value is } 1 .\end{array}$ & [65] \\
\hline & $\begin{array}{l}\text { Peak Signal to } \\
\text { Noise Ratio }\end{array}$ & $P S N R=20 \log \left(\frac{255}{R M S E}\right)(d B)$ & $\begin{array}{l}\text { Represents the ratio between the maximum possible power of a signal and the } \\
\text { power of corrupting noise. The larger the value, the better the effect. It is not } \\
\text { absolutely proportional to the observation of the human eye, and has some } \\
\text { limitations. }\end{array}$ & [66] \\
\hline & $\begin{array}{l}\text { Root Mean } \\
\text { Squared Error }\end{array}$ & $R M S E=\sqrt{\left(\sum_{i=1}^{M} \sum_{j=1}^{N}(I(i, j)-\hat{I}(i, j))^{2}\right) /(M \times N)}$ & $\begin{array}{l}\text { Computes the difference between the predicted value. In general, it is directly } \\
\text { used in PSNR. As can be seen from the formula, it is inversely proportional } \\
\text { to PSNR. }\end{array}$ & [67] \\
\hline & $\begin{array}{l}\text { Mean Operating } \\
\text { Time }\end{array}$ & Time $=\frac{\sum_{i=1}^{N} \text { time }}{N}$ & $\begin{array}{l}\text { The computational complexity is evaluated by experimental data. Average the } \\
\text { execution time of each algorithm running independently for } 30 \text { times. } \\
\text { The smaller the numerical value, the faster the algorithm is executed and the } \\
\text { lower the computational complexity. }\end{array}$ & [68] \\
\hline & $\begin{array}{l}\text { Average fitness } \\
\text { function value }\end{array}$ & Fitness $=\frac{\sum_{i=1}^{N} f_{i}}{N}$ & $\begin{array}{l}\text { The mathematical concept of optimization is the method of calculating the } \\
\text { value of a function and finding the optimal result by maximizing and } \\
\text { minimizing an objective function in a given domain. Therefore, the average } \\
\text { fitness value obtained through multiple measurements can be used to evaluate } \\
\text { the optimization results. }\end{array}$ & [69] \\
\hline \multirow{2}{*}{$\begin{array}{l}\text { Statistical } \\
\text { evaluation }\end{array}$} & $\begin{array}{l}\text { Wilcoxon's } \\
\text { Rank-Sum test }\end{array}$ & $\begin{aligned} R^{+} & =\sum_{d_{i}>0} \operatorname{rank}\left(d_{i}\right)+\frac{1}{1} \sum_{d_{i}=0} \operatorname{rank}\left(d_{i}\right) \\
R^{-} & =\sum_{d_{i}<0} \operatorname{rank}\left(d_{i}\right)+\frac{1}{2} \sum_{d_{i}=0} \operatorname{rank}\left(d_{i}\right)\end{aligned}$ & $\begin{array}{l}\text { Used to answer the question "Does two samples represent two different } \\
\text { populations?" In this paper, it is used to compare the difference between the } \\
\text { proposed algorithm and the comparison algorithm. If the } p \text {-value }>0.05 \\
\text { (or } \mathrm{h}=1 \text { ), there is a significant difference, otherwise it is not. }\end{array}$ & [70] \\
\hline & Friedman test & $F_{f}=\frac{12 n}{k(k+1)}\left[\sum_{j} R_{j}^{2}-\frac{k[k+1]^{2}}{4}\right]$ & $\begin{array}{l}\text { The Friedman test is a nonparametric simulation of nonparametric variance } \\
\text { bidirectional analysis. Used to answer the question "Does at least two samples } \\
\text { in a group of k samples represent populations with different median values?" } \\
\text { Designed to detect significant differences between the behavior of two or more } \\
\text { algorithms, the overall performance of the algorithm can be ranked. }\end{array}$ & [70] \\
\hline
\end{tabular}




\subsection{Implementation Results and Discussion}

In this section, the experimental results of the TLMVO-Masi multilevel threshold are described and analyzed in detail. According to the above metrics, it is analyzed from three aspects: image performance indicators, segmentation function performance index and mathematical statistics of data results. The superiority of TLMVO algorithm over other effective algorithms is verified. The following is a sub-section discussion.

\subsubsection{Image Segmentation Quality}

Measuring the performance by intensity and accuracy, Structural Similarity Index (SSIM), Feature Similarity Index (FSIM), and Peak Signal to Noise Ratio (PSNR) are ultilized. Segmentation effects of 10 satellite images are shown in Figure 6. Different thresholds of different algorithms of an image run 30 times, and then correspond to three indexes. The overall data space size is $3 \times 10 \times 8 \times 5=1200$ (10 images, 8 algorithms, 5 thresholds). Average data results of 30 times are indicated in Tables 5-7, respectively. The higher the similarity between the original image and the segmented image, the greater the value (the maximum value of SSIM and FSIM is 1). The maximum value of the index corresponding to each threshold is indicated by a distinct mark in tables.

These three tables clearly show that, as the threshold value increases, the value of the indicator also increases. This indicates that the segmentation quality is improved with the increase of threshold number, which can also correspond to the segmentation renderings we have given. As the threshold value increases, the segmentation image becomes clearer.

From the label distribution, it can be seen that most of the three index values of the proposed algorithm are more outstanding than comparison algorithms. For instance, in the case of various thresholds of TLMVO:

1. In the SSIM table: the values of Image 1 and Image 3 are all higher than the comparison algorithm;

2. In the FSIM table: Image 3 and Image 6 yield excellence values compared with other algorithms in all cases;

3. In the PSNR table: the values in Image 1, Image 3, and Image 8 are all much better than the comparative algorithms.

4

6

8

10

12

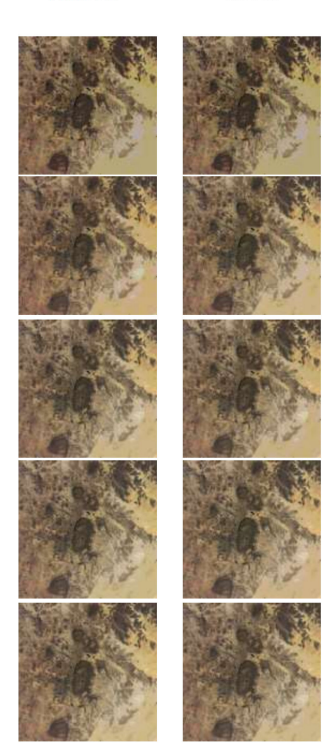

GSA

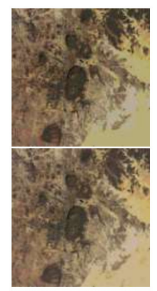

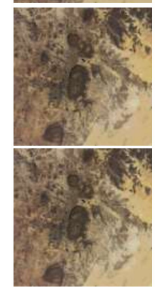

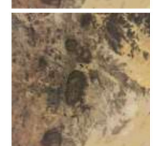

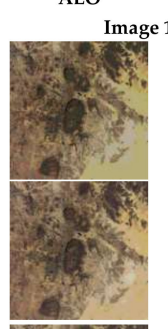
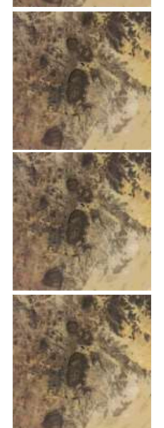
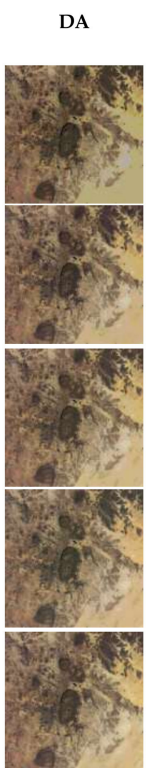

FPA
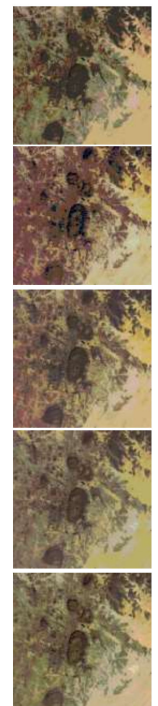

PSO
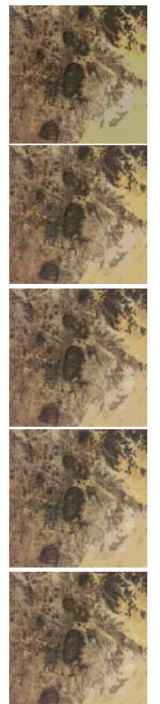

CS

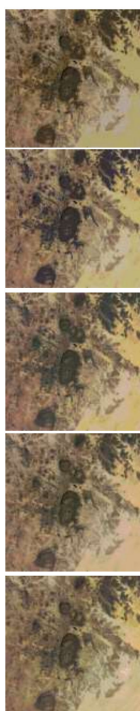

Figure 6. Cont. 

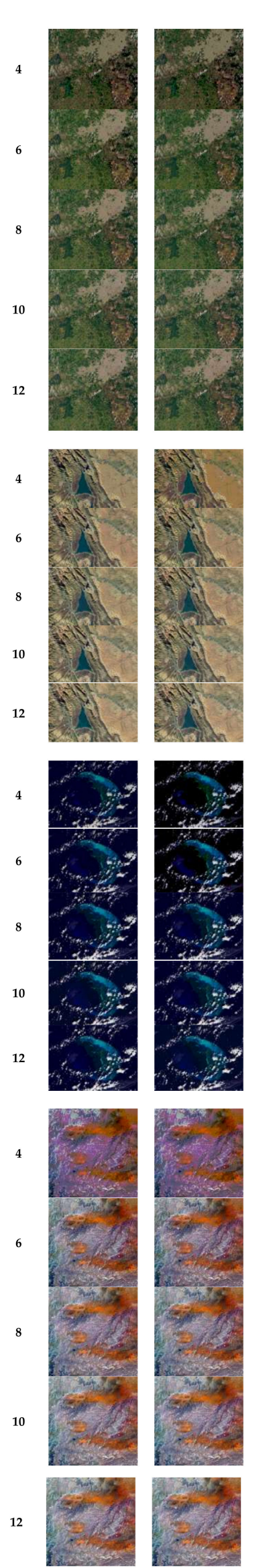
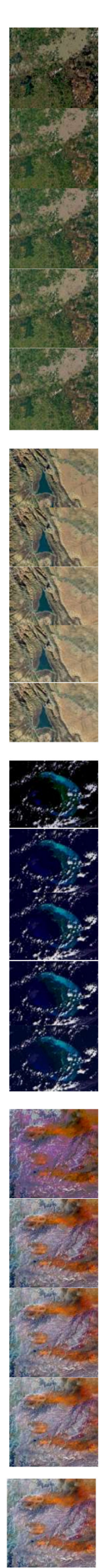
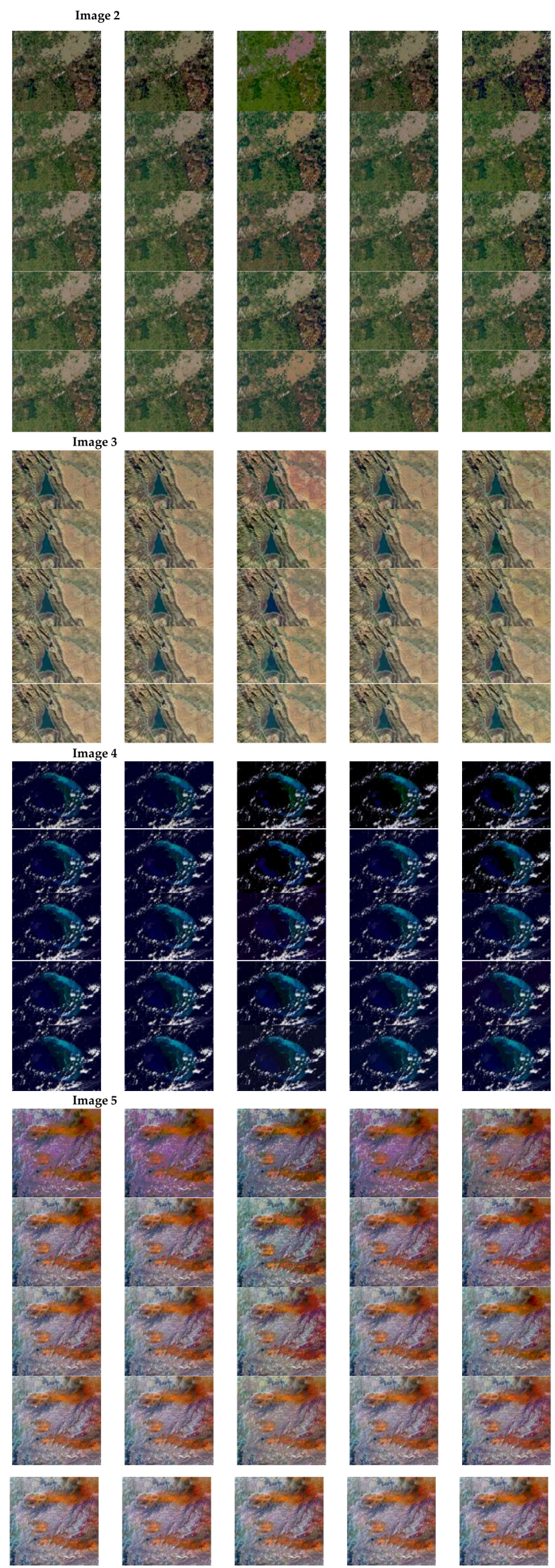

Figure 6. Cont. 


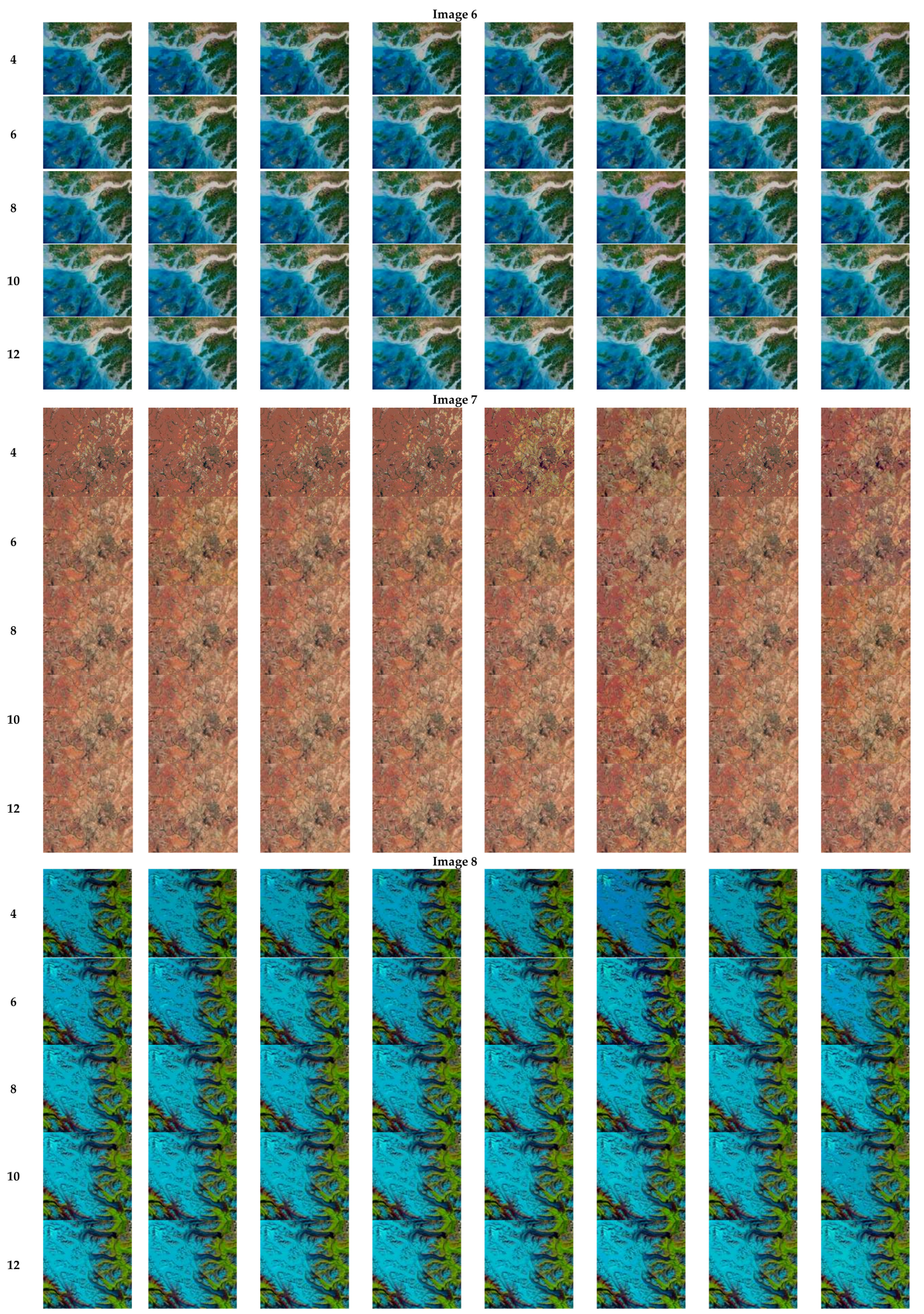

Figure 6. Cont. 

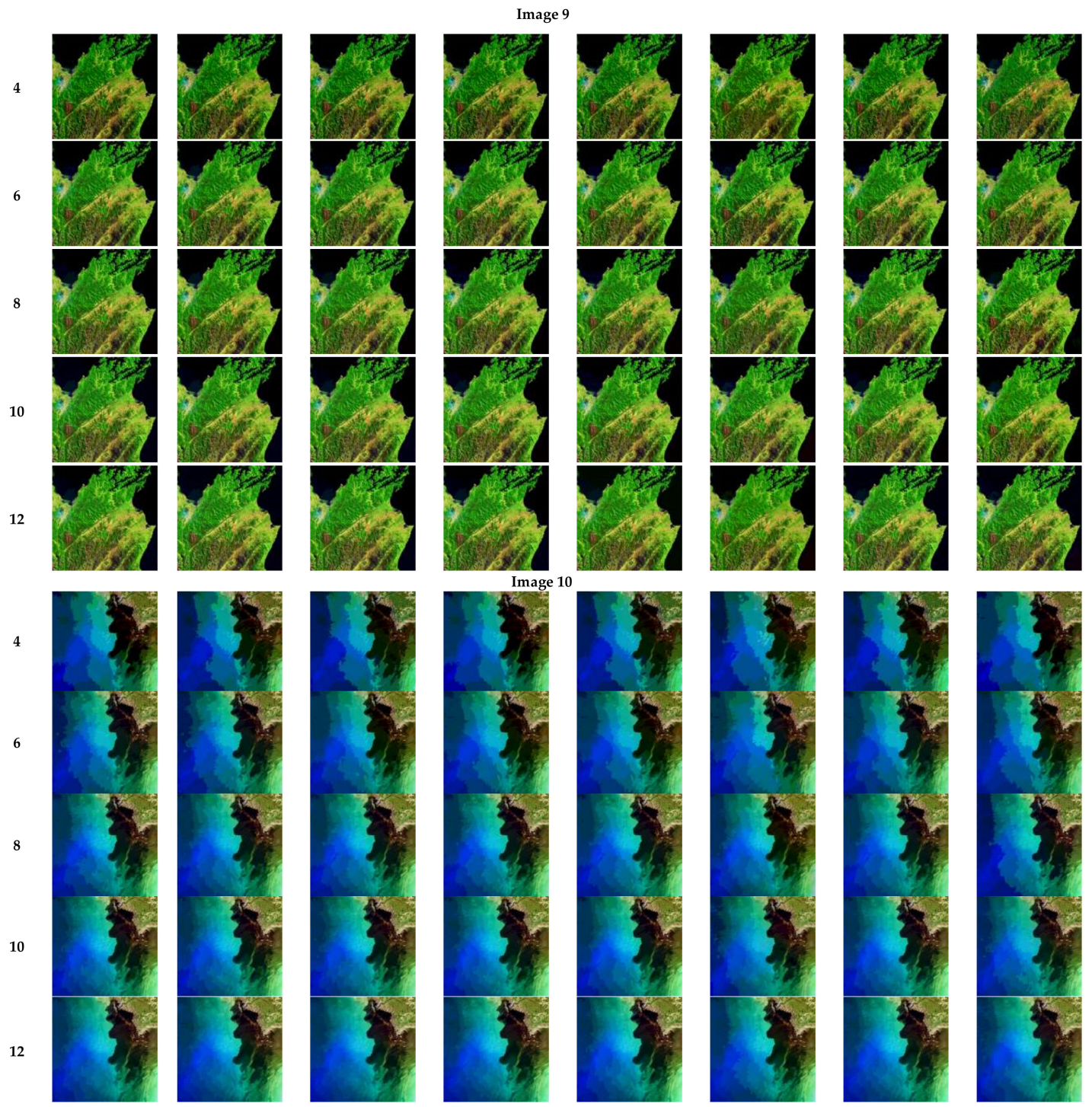

Figure 6. Segmentation renderings of 10 satellite images.

In order to more clearly see the superiority of TLMVO, the three index values of each test image are integrated into three line graphs (as shown in Figures 7-9). The data of TLMVO algorithm are represented in green. The green line can include other colored lines. To some extent, the results confirm that the improved algorithm can achieve good image segmentation quality. 
Table 5. The SSIM of each algorithm under Masi entropy.

\begin{tabular}{|c|c|c|c|c|c|c|c|c|c|}
\hline $\begin{array}{l}\text { TEST } \\
\text { IMAGES }\end{array}$ & $\mathbf{K}$ & TLMVO & LMVO & MVO & ALO & DA & FPA & PSO & CS \\
\hline \multirow{5}{*}{1} & 4 & 0.7744 & 0.7715 & 0.7740 & 0.7715 & 0.7723 & 0.7502 & 0.7715 & 0.7512 \\
\hline & 6 & 0.8566 & 0.8559 & 0.8558 & 0.8467 & 0.8556 & 0.7868 & 0.8408 & 0.8337 \\
\hline & 8 & 0.8994 & 0.8965 & 0.8862 & 0.8984 & 0.8923 & 0.8279 & 0.8971 & 0.8819 \\
\hline & 10 & 0.9296 & 0.9291 & 0.9239 & 0.9293 & 0.9278 & 0.8780 & 0.9262 & 0.8645 \\
\hline & 12 & 0.9438 & 0.9423 & 0.9413 & 0.9435 & 0.9393 & 0.8903 & 0.9383 & 0.9115 \\
\hline \multirow{5}{*}{2} & 4 & 0.7122 & 0.6452 & 0.6872 & 0.6855 & 0.6909 & 0.6702 & 0.6150 & 0.6914 \\
\hline & 6 & 0.8349 & 0.8151 & 0.8535 & 0.8542 & 0.8477 & 0.7471 & 0.8299 & 0.7989 \\
\hline & 8 & 0.9135 & 0.8964 & 0.8888 & 0.9052 & 0.8895 & 0.8649 & 0.8903 & 0.8605 \\
\hline & 10 & 0.9408 & 0.9317 & 0.9259 & 0.9271 & 0.9257 & 0.8708 & 0.9357 & 0.8901 \\
\hline & 12 & 0.9547 & 0.9489 & 0.9468 & 0.9528 & 0.9519 & 0.9070 & 0.9443 & 0.9265 \\
\hline \multirow{5}{*}{3} & 4 & 0.7959 & 0.7898 & 0.7830 & 0.7897 & 0.7914 & 0.7027 & 0.7502 & 0.7826 \\
\hline & 6 & 0.8726 & 0.8714 & 0.8694 & 0.8590 & 0.8650 & 0.8523 & 0.8654 & 0.8605 \\
\hline & 8 & 0.9133 & 0.9093 & 0.9085 & 0.9126 & 0.9101 & 0.8881 & 0.9091 & 0.8827 \\
\hline & 10 & 0.9415 & 0.9383 & 0.9386 & 0.9399 & 0.9404 & 0.8944 & 0.9383 & 0.9236 \\
\hline & 12 & 0.9580 & 0.9533 & 0.9537 & 0.9552 & 0.9529 & 0.9128 & 0.9569 & 0.9359 \\
\hline \multirow{5}{*}{4} & 4 & 0.4083 & 0.3809 & 0.3809 & 0.3762 & 0.3805 & 0.3674 & 0.3762 & 0.3791 \\
\hline & 6 & 0.6019 & 0.6002 & 0.5926 & 0.5929 & 0.5910 & 0.5836 & 0.5919 & 0.4427 \\
\hline & 8 & 0.6364 & 0.6362 & 0.6364 & 0.6195 & 0.6120 & 0.5571 & 0.6169 & 0.5880 \\
\hline & 10 & 0.7692 & 0.6526 & 0.6575 & 0.6532 & 0.6446 & 0.7614 & 0.7666 & 0.6435 \\
\hline & 12 & 0.7736 & 0.6744 & 0.6741 & 0.6649 & 0.6616 & 0.6895 & 0.7734 & 0.6183 \\
\hline \multirow{5}{*}{5} & 4 & 0.6682 & 0.6681 & 0.6239 & 0.6429 & 0.6271 & 0.6573 & 0.6430 & 0.6239 \\
\hline & 6 & 0.8053 & 0.8133 & 0.8022 & 0.8142 & 0.8074 & 0.7861 & 0.8034 & 0.7772 \\
\hline & 8 & 0.8819 & 0.8804 & 0.8786 & 0.8734 & 0.8739 & 0.8024 & 0.8789 & 0.8362 \\
\hline & 10 & 0.9142 & 0.9120 & 0.9115 & 0.9138 & 0.9104 & 0.8940 & 0.9119 & 0.8937 \\
\hline & 12 & 0.9420 & 0.9375 & 0.9348 & 0.9407 & 0.9416 & 0.8578 & 0.9402 & 0.9046 \\
\hline \multirow{5}{*}{6} & 4 & 0.7156 & 0.7156 & 0.7138 & 0.7138 & 0.7115 & 0.6512 & 0.7138 & 0.6914 \\
\hline & 6 & 0.8033 & 0.8084 & 0.8046 & 0.8032 & 0.8018 & 0.7928 & 0.8030 & 0.8075 \\
\hline & 8 & 0.8562 & 0.8548 & 0.8551 & 0.8550 & 0.8551 & 0.7803 & 0.8541 & 0.8022 \\
\hline & 10 & 0.8862 & 0.8847 & 0.8830 & 0.8802 & 0.8674 & 0.8332 & 0.8844 & 0.8738 \\
\hline & 12 & 0.9050 & 0.9007 & 0.8971 & 0.9037 & 0.8975 & 0.8567 & 0.9007 & 0.8661 \\
\hline \multirow{5}{*}{7} & 4 & 0.5752 & 0.6157 & 0.6152 & 0.6152 & 0.5965 & 0.5903 & 0.6046 & 0.5536 \\
\hline & 6 & 0.8252 & 0.7880 & 0.8201 & 0.8200 & 0.8244 & 0.7817 & 0.8250 & 0.8182 \\
\hline & 8 & 0.8907 & 0.8825 & 0.8756 & 0.8968 & 0.8775 & 0.8091 & 0.8789 & 0.8479 \\
\hline & 10 & 0.9294 & 0.9284 & 0.9185 & 0.9258 & 0.9228 & 0.7486 & 0.9249 & 0.8523 \\
\hline & 12 & 0.9507 & 0.9492 & 0.9449 & 0.9491 & 0.9412 & 0.8673 & 0.9502 & 0.9157 \\
\hline \multirow{5}{*}{8} & 4 & 0.6910 & 0.6999 & 0.6811 & 0.6999 & 0.6794 & 0.6687 & 0.6805 & 0.6399 \\
\hline & 6 & 0.7761 & 0.7766 & 0.7896 & 0.8021 & 0.7871 & 0.6895 & 0.7892 & 0.7106 \\
\hline & 8 & 0.8648 & 0.8616 & 0.8555 & 0.8565 & 0.8507 & 0.7228 & 0.8574 & 0.8287 \\
\hline & 10 & 0.8961 & 0.8959 & 0.8930 & 0.8952 & 0.8885 & 0.8764 & 0.8854 & 0.8154 \\
\hline & 12 & 0.9159 & 0.9126 & 0.9121 & 0.9104 & 0.8967 & 0.8502 & 0.9139 & 0.8714 \\
\hline \multirow{5}{*}{9} & 4 & 0.5578 & 0.5576 & 0.5435 & 0.5434 & 0.5434 & 0.5077 & 0.5575 & 0.5120 \\
\hline & 6 & 0.7024 & 0.7047 & 0.7059 & 0.6993 & 0.6940 & 0.7032 & 0.7016 & 0.6858 \\
\hline & 8 & 0.7578 & 0.7555 & 0.7470 & 0.7484 & 0.7380 & 0.6107 & 0.7480 & 0.6813 \\
\hline & 10 & 0.7967 & 0.7780 & 0.7825 & 0.7762 & 0.7789 & 0.7741 & 0.7771 & 0.6913 \\
\hline & 12 & 0.8625 & 0.8042 & 0.8506 & 0.8063 & 0.8382 & 0.7635 & 0.8393 & 0.7152 \\
\hline \multirow{5}{*}{10} & 4 & 0.6274 & 0.6237 & 0.6237 & 0.5968 & 0.6238 & 0.5912 & 0.6237 & 0.5927 \\
\hline & 6 & 0.7429 & 0.7001 & 0.7331 & 0.7412 & 0.7360 & 0.6911 & 0.7316 & 0.7057 \\
\hline & 8 & 0.7779 & 0.7829 & 0.7777 & 0.7757 & 0.7614 & 0.7353 & 0.7764 & 0.6985 \\
\hline & 10 & 0.8147 & 0.8143 & 0.8005 & 0.7991 & 0.7917 & 0.7798 & 0.7958 & 0.7905 \\
\hline & 12 & 0.8439 & 0.8406 & 0.8434 & 0.8361 & 0.8315 & 0.7940 & 0.8328 & 0.7981 \\
\hline
\end{tabular}


Table 6. The FSIM of each algorithm under Masi entropy.

\begin{tabular}{|c|c|c|c|c|c|c|c|c|c|}
\hline $\begin{array}{l}\text { TEST } \\
\text { IMAGES }\end{array}$ & $\mathbf{K}$ & TLMVO & LMVO & MVO & ALO & DA & FPA & PSO & CS \\
\hline \multirow{5}{*}{1} & 4 & 0.8408 & 0.8401 & 0.8379 & 0.8379 & 0.8389 & 0.8138 & 0.8379 & 0.8199 \\
\hline & 6 & 0.9109 & 0.9093 & 0.8870 & 0.9039 & 0.9089 & 0.8440 & 0.8990 & 0.8856 \\
\hline & 8 & 0.9332 & 0.9395 & 0.9416 & 0.9410 & 0.9352 & 0.8850 & 0.9405 & 0.9193 \\
\hline & 10 & 0.9572 & 0.9514 & 0.9607 & 0.9616 & 0.9599 & 0.9209 & 0.9597 & 0.9079 \\
\hline & 12 & 0.9697 & 0.9695 & 0.9671 & 0.9690 & 0.9684 & 0.9279 & 0.9672 & 0.9396 \\
\hline \multirow{5}{*}{2} & 4 & 0.8433 & 0.8398 & 0.8214 & 0.8279 & 0.8338 & 0.8218 & 0.7855 & 0.8108 \\
\hline & 6 & 0.9157 & 0.9055 & 0.9321 & 0.9331 & 0.9223 & 0.8618 & 0.9158 & 0.9019 \\
\hline & 8 & 0.9592 & 0.9534 & 0.9490 & 0.9556 & 0.9482 & 0.9216 & 0.9501 & 0.9306 \\
\hline & 10 & 0.9736 & 0.9692 & 0.9662 & 0.9672 & 0.9660 & 0.9298 & 0.9717 & 0.9369 \\
\hline & 12 & 0.9803 & 0.9735 & 0.9776 & 0.9791 & 0.9786 & 0.9433 & 0.9752 & 0.9570 \\
\hline \multirow{5}{*}{3} & 4 & 0.8967 & 0.8941 & 0.8935 & 0.8679 & 0.8962 & 0.8358 & 0.8723 & 0.8842 \\
\hline & 6 & 0.9447 & 0.9398 & 0.9439 & 0.9390 & 0.9398 & 0.9188 & 0.9420 & 0.9269 \\
\hline & 8 & 0.9641 & 0.9625 & 0.9587 & 0.9615 & 0.9605 & 0.9393 & 0.9610 & 0.9380 \\
\hline & 10 & 0.9751 & 0.9743 & 0.9743 & 0.9746 & 0.9741 & 0.9513 & 0.9738 & 0.9581 \\
\hline & 12 & 0.9816 & 0.9804 & 0.9801 & 0.9806 & 0.9799 & 0.9528 & 0.9812 & 0.9682 \\
\hline \multirow{5}{*}{4} & 4 & 0.8717 & 0.8640 & 0.8640 & 0.8630 & 0.8650 & 0.8492 & 0.8630 & 0.8595 \\
\hline & 6 & 0.8928 & 0.9137 & 0.9124 & 0.9130 & 0.9123 & 0.8932 & 0.9123 & 0.9062 \\
\hline & 8 & 0.9337 & 0.9335 & 0.9335 & 0.9312 & 0.9301 & 0.8839 & 0.9308 & 0.9115 \\
\hline & 10 & 0.9496 & 0.9453 & 0.9435 & 0.9452 & 0.9377 & 0.9219 & 0.9488 & 0.9255 \\
\hline & 12 & 0.9558 & 0.9536 & 0.9541 & 0.9533 & 0.9512 & 0.9200 & 0.9540 & 0.9299 \\
\hline \multirow{5}{*}{5} & 4 & 0.7343 & 0.7729 & 0.7343 & 0.7543 & 0.7427 & 0.7661 & 0.7540 & 0.7724 \\
\hline & 6 & 0.8878 & 0.8874 & 0.8805 & 0.8809 & 0.8817 & 0.8570 & 0.8807 & 0.8546 \\
\hline & 8 & 0.9335 & 0.9335 & 0.9347 & 0.9314 & 0.9302 & 0.8667 & 0.9332 & 0.8994 \\
\hline & 10 & 0.9552 & 0.9540 & 0.9537 & 0.9544 & 0.9515 & 0.9316 & 0.9536 & 0.9294 \\
\hline & 12 & 0.9703 & 0.9676 & 0.9668 & 0.9651 & 0.9695 & 0.9023 & 0.9694 & 0.9421 \\
\hline \multirow{5}{*}{6} & 4 & 0.8092 & 0.8092 & 0.8081 & 0.8081 & 0.8062 & 0.7641 & 0.8081 & 0.7926 \\
\hline & 6 & 0.8807 & 0.8754 & 0.8794 & 0.8794 & 0.8793 & 0.8494 & 0.8792 & 0.8724 \\
\hline & 8 & 0.9232 & 0.9171 & 0.9218 & 0.9219 & 0.9223 & 0.8545 & 0.9207 & 0.8690 \\
\hline & 10 & 0.9442 & 0.9438 & 0.9279 & 0.9415 & 0.9357 & 0.8981 & 0.9353 & 0.9289 \\
\hline & 12 & 0.9555 & 0.9545 & 0.9539 & 0.9551 & 0.9526 & 0.9145 & 0.9541 & 0.9186 \\
\hline \multirow{5}{*}{7} & 4 & 0.7974 & 0.7376 & 0.7369 & 0.7369 & 0.7212 & 0.7106 & 0.7284 & 0.7651 \\
\hline & 6 & 0.9064 & 0.8779 & 0.8985 & 0.8983 & 0.9014 & 0.8786 & 0.9053 & 0.8959 \\
\hline & 8 & 0.9495 & 0.9423 & 0.9379 & 0.9471 & 0.9402 & 0.8763 & 0.9399 & 0.9125 \\
\hline & 10 & 0.9646 & 0.9632 & 0.9685 & 0.9685 & 0.9671 & 0.8356 & 0.9667 & 0.9082 \\
\hline & 12 & 0.9785 & 0.9764 & 0.9756 & 0.9780 & 0.9743 & 0.9076 & 0.9736 & 0.9528 \\
\hline \multirow{5}{*}{8} & 4 & 0.8378 & 0.8395 & 0.8243 & 0.8395 & 0.8230 & 0.8101 & 0.8246 & 0.7957 \\
\hline & 6 & 0.8914 & 0.8904 & 0.9010 & 0.9078 & 0.8962 & 0.8173 & 0.9014 & 0.8249 \\
\hline & 8 & 0.9400 & 0.9360 & 0.9396 & 0.9385 & 0.9347 & 0.8508 & 0.9391 & 0.9090 \\
\hline & 10 & 0.9584 & 0.9554 & 0.9562 & 0.9582 & 0.9546 & 0.9211 & 0.9538 & 0.9145 \\
\hline & 12 & 0.9686 & 0.9675 & 0.9666 & 0.9662 & 0.9632 & 0.9262 & 0.9681 & 0.9396 \\
\hline \multirow{5}{*}{9} & 4 & 0.8541 & 0.8534 & 0.8473 & 0.8472 & 0.8472 & 0.8278 & 0.8534 & 0.8221 \\
\hline & 6 & 0.9285 & 0.9276 & 0.9283 & 0.9277 & 0.9249 & 0.8928 & 0.9274 & 0.9081 \\
\hline & 8 & 0.9502 & 0.9487 & 0.9487 & 0.9485 & 0.9466 & 0.8913 & 0.9482 & 0.9213 \\
\hline & 10 & 0.9585 & 0.9618 & 0.9588 & 0.9590 & 0.9580 & 0.9402 & 0.9575 & 0.9241 \\
\hline & 12 & 0.9747 & 0.9709 & 0.9658 & 0.9666 & 0.9708 & 0.9292 & 0.9715 & 0.9377 \\
\hline \multirow{5}{*}{10} & 4 & 0.7640 & 0.7640 & 0.7599 & 0.7634 & 0.7640 & 0.7528 & 0.7599 & 0.7514 \\
\hline & 6 & 0.8242 & 0.8210 & 0.8240 & 0.8250 & 0.8226 & 0.8073 & 0.8244 & 0.8015 \\
\hline & 8 & 0.8620 & 0.8598 & 0.8596 & 0.8592 & 0.8478 & 0.8242 & 0.8592 & 0.8300 \\
\hline & 10 & 0.8917 & 0.8837 & 0.8834 & 0.8829 & 0.8762 & 0.8494 & 0.8810 & 0.8619 \\
\hline & 12 & 0.9113 & 0.9092 & 0.9108 & 0.9084 & 0.9036 & 0.8819 & 0.9059 & 0.8802 \\
\hline
\end{tabular}


Table 7. The PSNR of each algorithm under Masi entropy.

\begin{tabular}{|c|c|c|c|c|c|c|c|c|c|}
\hline $\begin{array}{l}\text { TEST } \\
\text { IMAGES }\end{array}$ & $\mathbf{K}$ & TLMVO & LMVO & MVO & ALO & DA & FPA & PSO & CS \\
\hline \multirow{5}{*}{1} & 4 & 20.7925 & 20.7725 & 20.7675 & 20.7125 & 20.7915 & 20.2532 & 20.7125 & 20.1630 \\
\hline & 6 & 24.1576 & 23.4460 & 24.1240 & 23.7732 & 24.1316 & 20.8999 & 22.9504 & 22.8607 \\
\hline & 8 & 26.2592 & 26.0185 & 25.5235 & 26.2024 & 25.9712 & 23.5785 & 26.1342 & 25.3450 \\
\hline & 10 & 28.3717 & 28.1962 & 28.1737 & 28.2740 & 28.2269 & 25.6046 & 28.0548 & 24.8368 \\
\hline & 12 & 29.6100 & 29.3884 & 29.3109 & 29.6050 & 29.2210 & 26.4913 & 29.0708 & 27.2000 \\
\hline \multirow{5}{*}{2} & 4 & 18.8430 & 18.8249 & 18.6134 & 18.9307 & 18.9290 & 18.2521 & 17.2951 & 18.1679 \\
\hline & 6 & 22.3266 & 21.6960 & 22.8578 & 22.8863 & 22.5439 & 20.4732 & 22.1872 & 21.1393 \\
\hline & 8 & 24.2476 & 24.6675 & 25.3874 & 24.9641 & 24.3533 & 23.5434 & 24.3869 & 23.5563 \\
\hline & 10 & 27.2892 & 27.2621 & 27.2874 & 26.3446 & 26.2204 & 24.1414 & 26.8406 & 24.8671 \\
\hline & 12 & 28.5535 & 27.9996 & 27.9939 & 28.3447 & 28.3496 & 25.8873 & 27.6342 & 26.8304 \\
\hline \multirow{5}{*}{3} & 4 & 19.9262 & 18.8001 & 19.6044 & 18.8017 & 19.8648 & 17.8319 & 18.9799 & 19.5485 \\
\hline & 6 & 22.6252 & 22.3173 & 22.5656 & 22.2449 & 22.6239 & 21.8502 & 22.4853 & 22.4241 \\
\hline & 8 & 24.7823 & 24.5854 & 24.5670 & 24.7439 & 24.3765 & 23.6344 & 24.2951 & 23.1364 \\
\hline & 10 & 26.4116 & 26.3248 & 26.3246 & 26.4026 & 26.3820 & 24.7313 & 25.5045 & 25.7397 \\
\hline & 12 & 28.2060 & 27.6567 & 27.8359 & 27.8759 & 27.7598 & 25.1138 & 28.0180 & 26.7576 \\
\hline \multirow{5}{*}{4} & 4 & 21.7947 & 19.5676 & 19.5676 & 19.5394 & 19.5675 & 19.2235 & 19.5394 & 19.5386 \\
\hline & 6 & 21.0832 & 23.8521 & 23.7218 & 23.7330 & 23.6856 & 22.5114 & 23.7101 & 23.4854 \\
\hline & 8 & 25.0875 & 25.0789 & 25.0852 & 24.8347 & 24.7279 & 23.2739 & 24.8134 & 23.3235 \\
\hline & 10 & 27.1861 & 26.1187 & 25.9677 & 26.1052 & 25.4995 & 25.7035 & 27.0715 & 25.3684 \\
\hline & 12 & 27.4950 & 26.9966 & 26.9420 & 26.9006 & 26.5768 & 25.5771 & 26.7394 & 24.9418 \\
\hline \multirow{5}{*}{5} & 4 & 16.9806 & 17.8866 & 16.9806 & 17.4367 & 16.8512 & 17.2823 & 17.4483 & 17.8100 \\
\hline & 6 & 21.3662 & 21.1859 & 20.9164 & 21.2681 & 21.0219 & 20.6315 & 20.9578 & 20.5071 \\
\hline & 8 & 23.5702 & 23.6344 & 23.6781 & 23.4792 & 23.3843 & 21.1273 & 23.5965 & 22.0975 \\
\hline & 10 & 25.3535 & 25.3249 & 25.3250 & 25.3295 & 25.1868 & 24.7880 & 25.2063 & 24.7622 \\
\hline & 12 & 26.2153 & 26.9166 & 26.6882 & 27.1381 & 27.3483 & 23.7282 & 27.1303 & 25.8037 \\
\hline \multirow{5}{*}{6} & 4 & 20.5027 & 20.5027 & 20.4732 & 20.4732 & 20.4333 & 19.0972 & 20.4732 & 19.9978 \\
\hline & 6 & 23.4533 & 23.4390 & 23.4334 & 23.4054 & 23.4079 & 21.9773 & 23.3958 & 23.1176 \\
\hline & 8 & 25.9008 & 25.8499 & 25.8486 & 25.8400 & 25.8486 & 22.5025 & 25.7739 & 23.2438 \\
\hline & 10 & 26.9624 & 26.5210 & 27.5186 & 27.2226 & 26.6858 & 24.7022 & 27.4974 & 26.6856 \\
\hline & 12 & 28.4770 & 28.5111 & 28.4938 & 28.5166 & 28.1274 & 25.8854 & 28.3971 & 26.2981 \\
\hline \multirow{5}{*}{7} & 4 & 17.3110 & 17.3778 & 17.3814 & 17.3814 & 17.1450 & 17.2728 & 17.1777 & 17.2192 \\
\hline & 6 & 22.1716 & 21.8913 & 21.8272 & 22.0566 & 21.9270 & 20.5434 & 21.9065 & 21.8887 \\
\hline & 8 & 24.8042 & 24.0714 & 23.9957 & 24.7965 & 23.9964 & 21.9582 & 24.1262 & 23.0275 \\
\hline & 10 & 26.6863 & 26.3535 & 26.0397 & 26.6338 & 26.4948 & 21.6290 & 26.4367 & 24.1686 \\
\hline & 12 & 28.3561 & 28.3134 & 27.9492 & 28.3138 & 27.6726 & 24.4441 & 27.6680 & 26.2470 \\
\hline \multirow{5}{*}{8} & 4 & 20.0461 & 20.0461 & 19.4775 & 20.0461 & 19.4719 & 19.5537 & 19.4627 & 19.0435 \\
\hline & 6 & 23.2358 & 22.7455 & 22.7302 & 23.0977 & 22.4261 & 19.4761 & 22.7791 & 19.9978 \\
\hline & 8 & 25.2157 & 25.1023 & 24.7838 & 25.2140 & 24.9005 & 21.5724 & 25.1973 & 23.3424 \\
\hline & 10 & 27.3115 & 26.7463 & 26.7323 & 27.2165 & 26.7850 & 25.1312 & 26.6258 & 24.5564 \\
\hline & 12 & 28.4387 & 28.3717 & 28.2705 & 28.3854 & 28.0137 & 25.4779 & 28.4350 & 27.1038 \\
\hline \multirow{5}{*}{9} & 4 & 19.2111 & 19.1843 & 19.0203 & 19.0148 & 19.0148 & 18.4891 & 19.1816 & 18.8663 \\
\hline & 6 & 22.8662 & 22.7671 & 22.8598 & 22.6730 & 22.5579 & 21.6290 & 22.7617 & 22.0944 \\
\hline & 8 & 24.6355 & 24.6272 & 24.7685 & 24.5523 & 24.3009 & 21.5557 & 24.5443 & 23.4442 \\
\hline & 10 & 26.1033 & 26.3495 & 26.2421 & 26.0171 & 25.9568 & 24.6928 & 25.7938 & 23.8834 \\
\hline & 12 & 28.1868 & 27.7538 & 27.5842 & 27.2778 & 27.9255 & 24.1660 & 27.0234 & 24.3194 \\
\hline \multirow{5}{*}{10} & 4 & 18.5418 & 18.8095 & 18.5418 & 19.9601 & 18.8095 & 19.7825 & 18.5418 & 19.6373 \\
\hline & 6 & 22.9894 & 22.6055 & 22.8154 & 22.9843 & 22.8644 & 22.2684 & 22.8347 & 21.0550 \\
\hline & 8 & 25.2811 & 25.5629 & 25.3434 & 25.2261 & 24.4932 & 23.5053 & 25.2552 & 22.7493 \\
\hline & 10 & 27.3663 & 27.3658 & 26.7641 & 26.7074 & 26.1830 & 24.0665 & 26.5807 & 25.4793 \\
\hline & 12 & 28.8324 & 28.6704 & 28.6576 & 28.6044 & 28.0206 & 27.0101 & 28.3926 & 27.1410 \\
\hline
\end{tabular}




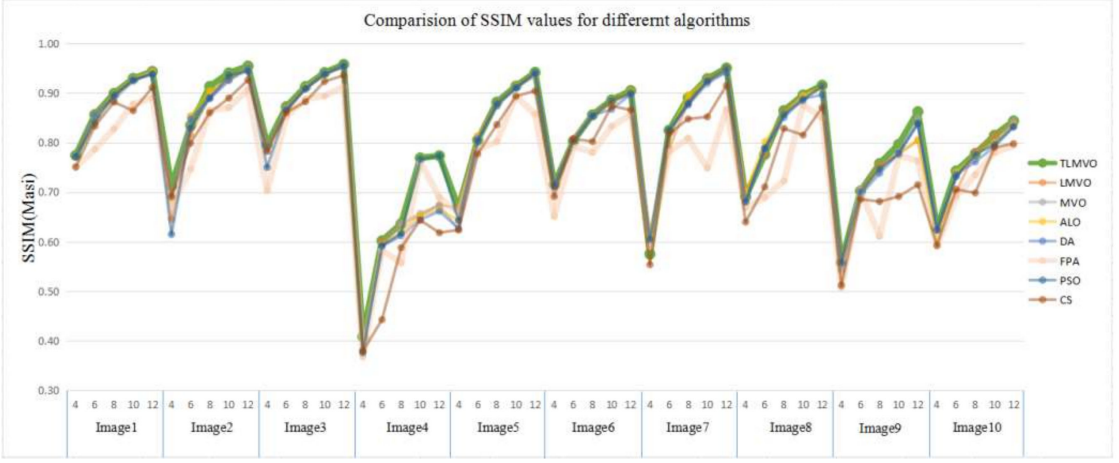

Figure 7. Broken line chart of SSIM indicator.

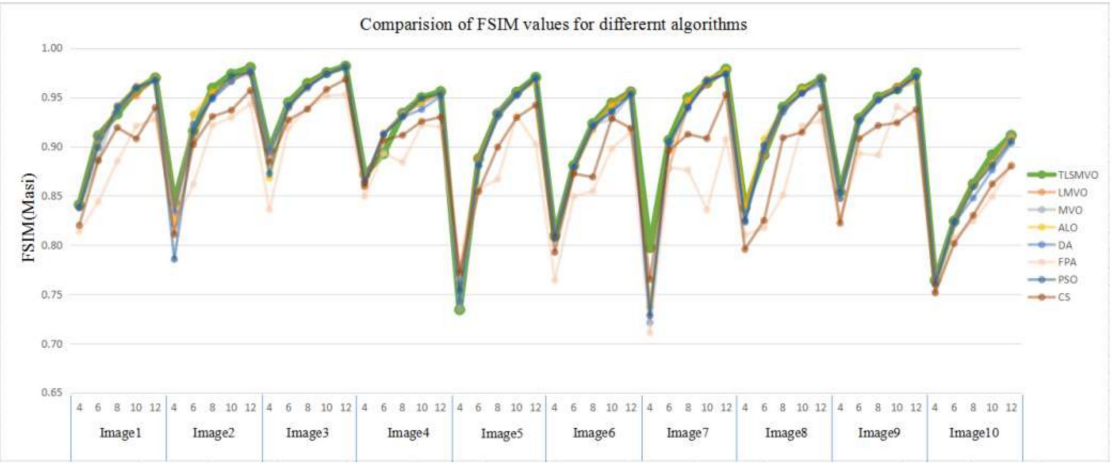

Figure 8. Broken line chart of FSIM indicator.

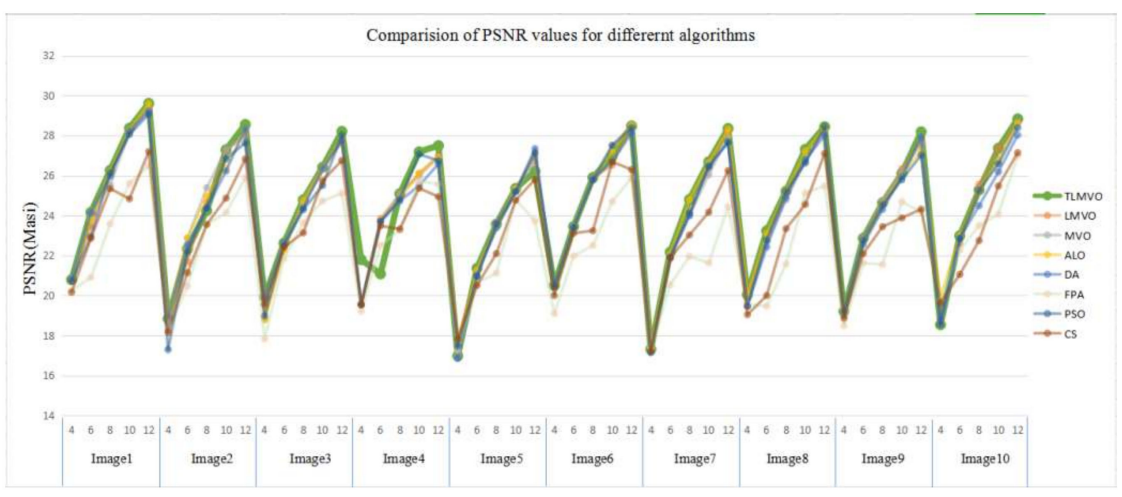

Figure 9. Broken line chart of FSIM indicator.

\subsubsection{Fitness Function Value Analysis}

A Masi entropy couple with an optimization algorithm to image segmentation is to take Masi entropy function as the fitness function of optimization. Therefore, the fitness function value can be a major concern to evaluate the performance of algorithm.

Table 8 is the average value of the fitness function values obtained by the TLMVO-Masi method for 30 times, wherein the maximum value of fitness functions corresponding to various threshold values are expressed by adding shadows. Under the condition of the maximum threshold, the convergence curves of the TLMVO algorithm compared with other algorithms are shown in Figure 10, which is mainly used for the analysis of convergence speed and robustness. The convergence curve of TLMVO is marked in red for distinction. In order to make a visual observation, furthermore, box plots corresponding to each set of convergence curves are produced to consolidate the judgment on the stability of the algorithm. 
Table 8. The optimal fitness value of each algorithm under Masi entropy.

\begin{tabular}{|c|c|c|c|c|c|c|c|c|c|}
\hline $\begin{array}{l}\text { TEST } \\
\text { IMAGES }\end{array}$ & $\mathbf{K}$ & TLMVO & LMVO & MVO & ALO & DA & FPA & PSO & CS \\
\hline \multirow{5}{*}{1} & 4 & 26.7841 & 26.7829 & 26.7759 & 26.7829 & 26.7814 & 26.5055 & 26.7829 & 26.5970 \\
\hline & 6 & 31.5270 & 32.3884 & 32.4733 & 32.4287 & 32.4701 & 30.8250 & 32.4650 & 31.7859 \\
\hline & 8 & 37.4559 & 37.4196 & 37.4012 & 37.4534 & 37.3572 & 34.7005 & 36.4051 & 36.3904 \\
\hline & 10 & 41.7545 & 40.3203 & 41.6523 & 41.7150 & 41.6303 & 37.9999 & 41.5054 & 39.5423 \\
\hline & 12 & 45.4460 & 45.4398 & 45.2587 & 45.4081 & 45.4063 & 42.1282 & 44.2942 & 42.7526 \\
\hline \multirow{5}{*}{2} & 4 & 28.6708 & 28.6708 & 28.6708 & 28.6395 & 28.6330 & 27.8866 & 28.6523 & 28.3837 \\
\hline & 6 & 34.8390 & 34.7560 & 34.8757 & 34.8782 & 34.8344 & 33.5580 & 34.8406 & 34.3164 \\
\hline & 8 & 40.2542 & 40.1957 & 40.1659 & 40.2540 & 40.2045 & 38.0999 & 40.2133 & 39.6369 \\
\hline & 10 & 45.0650 & 45.0265 & 44.9409 & 45.0126 & 44.8228 & 42.3067 & 44.8578 & 43.0264 \\
\hline & 12 & 49.1601 & 49.0709 & 48.9234 & 49.1108 & 49.0990 & 45.7133 & 48.5453 & 46.3682 \\
\hline \multirow{5}{*}{3} & 4 & 29.7577 & 29.7323 & 29.7778 & 29.7830 & 29.7760 & 29.4473 & 29.7831 & 29.6724 \\
\hline & 6 & 36.0540 & 36.0462 & 36.0337 & 36.0490 & 36.0312 & 35.1721 & 36.0420 & 35.4629 \\
\hline & 8 & 41.4123 & 41.4249 & 41.4764 & 41.4795 & 41.4817 & 40.0575 & 41.4816 & 40.2871 \\
\hline & 10 & 46.3981 & 46.3759 & 46.3513 & 46.3908 & 46.3270 & 43.8415 & 46.3492 & 44.9901 \\
\hline & 12 & 50.6654 & 50.6416 & 50.5188 & 50.0293 & 50.6070 & 48.5678 & 50.6194 & 48.8457 \\
\hline \multirow{5}{*}{4} & 4 & 31.8638 & 31.8631 & 31.8631 & 31.8638 & 31.8628 & 31.5520 & 31.8638 & 31.7064 \\
\hline & 6 & 38.4658 & 38.4457 & 38.3229 & 38.4712 & 38.4541 & 37.6742 & 38.4701 & 38.0972 \\
\hline & 8 & 44.1409 & 44.1299 & 44.1215 & 44.1379 & 44.1130 & 41.8809 & 44.1281 & 43.0865 \\
\hline & 10 & 49.1757 & 49.1380 & 49.0402 & 49.1403 & 48.9261 & 46.7316 & 49.0453 & 47.3696 \\
\hline & 12 & 53.6061 & 53.5976 & 53.5837 & 53.5429 & 53.4373 & 50.2012 & 52.8905 & 51.2963 \\
\hline \multirow{5}{*}{5} & 4 & 31.2635 & 31.2635 & 31.2635 & 31.2674 & 31.2400 & 30.9963 & 31.2667 & 31.0917 \\
\hline & 6 & 38.0624 & 38.0590 & 38.0595 & 38.0481 & 38.0599 & 37.0511 & 38.0598 & 37.6129 \\
\hline & 8 & 43.9486 & 43.9541 & 43.9283 & 43.9474 & 43.9390 & 42.3299 & 43.9476 & 43.0948 \\
\hline & 10 & 49.0856 & 49.0812 & 49.0386 & 49.0735 & 49.0009 & 46.9090 & 48.9981 & 46.9546 \\
\hline & 12 & 53.6589 & 53.6476 & 53.5966 & 53.6480 & 53.5644 & 51.3290 & 53.4092 & 51.4370 \\
\hline \multirow{5}{*}{6} & 4 & 30.1881 & 30.1881 & 30.1884 & 30.1884 & 30.1868 & 29.8118 & 30.1884 & 30.0943 \\
\hline & 6 & 36.3190 & 36.3116 & 36.3166 & 36.3189 & 36.3107 & 35.2884 & 36.3189 & 35.7087 \\
\hline & 8 & 41.5411 & 41.5357 & 41.4566 & 41.5381 & 41.5376 & 39.0273 & 41.5191 & 40.3121 \\
\hline & 10 & 46.1437 & 46.1336 & 45.8943 & 46.1245 & 46.0484 & 44.1846 & 46.1421 & 45.1448 \\
\hline & 12 & 50.4025 & 50.3495 & 49.7042 & 50.4011 & 50.3494 & 46.8572 & 50.0727 & 47.8994 \\
\hline \multirow{5}{*}{7} & 4 & 28.2182 & 28.3445 & 28.3453 & 28.3453 & 28.3106 & 27.5565 & 28.3456 & 28.0657 \\
\hline & 6 & 35.0028 & 34.9839 & 34.9229 & 34.9842 & 34.9778 & 33.8109 & 34.9850 & 34.4786 \\
\hline & 8 & 40.6323 & 40.4641 & 40.6104 & 40.5998 & 40.5242 & 38.6748 & 40.6264 & 39.6272 \\
\hline & 10 & 45.4981 & 45.4799 & 45.4692 & 45.4722 & 45.3232 & 42.7771 & 44.7652 & 43.0443 \\
\hline & 12 & 49.7507 & 49.5460 & 49.5312 & 49.7327 & 49.6270 & 45.9063 & 49.4327 & 46.5936 \\
\hline \multirow{5}{*}{8} & 4 & 30.6144 & 30.6455 & 30.6434 & 30.6355 & 30.6434 & 30.0874 & 30.6435 & 30.4565 \\
\hline & 6 & 37.0419 & 37.0032 & 37.0391 & 37.0385 & 37.0017 & 35.5762 & 37.0027 & 36.3631 \\
\hline & 8 & 42.5924 & 42.5407 & 42.5793 & 42.5912 & 42.4962 & 40.5814 & 42.5771 & 41.4359 \\
\hline & 10 & 47.2110 & 47.1791 & 47.2474 & 47.3335 & 47.3048 & 45.1692 & 46.6687 & 45.1569 \\
\hline & 12 & 51.6627 & 50.9570 & 51.0226 & 51.6421 & 51.0024 & 47.7623 & 51.6543 & 49.7426 \\
\hline \multirow{5}{*}{9} & 4 & 30.2650 & 30.2650 & 30.2647 & 30.2650 & 30.2650 & 29.7961 & 30.2615 & 29.9921 \\
\hline & 6 & 36.5929 & 36.5894 & 36.5848 & 36.5924 & 36.5903 & 34.7929 & 36.5877 & 35.9927 \\
\hline & 8 & 42.0890 & 42.0789 & 42.0739 & 42.0757 & 42.0501 & 40.7925 & 42.0803 & 41.0783 \\
\hline & 10 & 46.8554 & 46.7928 & 46.0774 & 46.8408 & 46.8110 & 44.5693 & 46.7586 & 45.2086 \\
\hline & 12 & 51.1184 & 50.9838 & 50.9699 & 50.5265 & 51.0424 & 47.4544 & 50.9939 & 48.8786 \\
\hline \multirow{5}{*}{10} & 4 & 32.3191 & 32.3127 & 32.3191 & 32.2953 & 32.3127 & 31.8869 & 32.3191 & 32.0323 \\
\hline & 6 & 38.9318 & 38.8767 & 38.9250 & 38.9300 & 38.9223 & 37.9559 & 38.9258 & 38.3460 \\
\hline & 8 & 44.6486 & 44.5368 & 44.6408 & 44.6440 & 44.4628 & 42.9020 & 44.6292 & 43.5905 \\
\hline & 10 & 49.6640 & 49.5103 & 49.5058 & 49.6494 & 49.5299 & 47.0386 & 49.6353 & 48.4979 \\
\hline & 12 & 54.1631 & 54.1038 & 54.0213 & 54.1207 & 54.0565 & 52.1343 & 54.0872 & 52.3326 \\
\hline
\end{tabular}




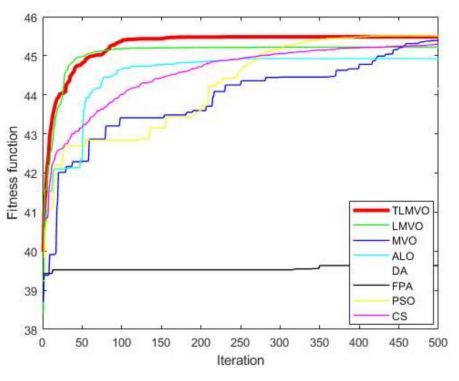

(a1) The values of Masi entropy unde eight algorithms in Image 1

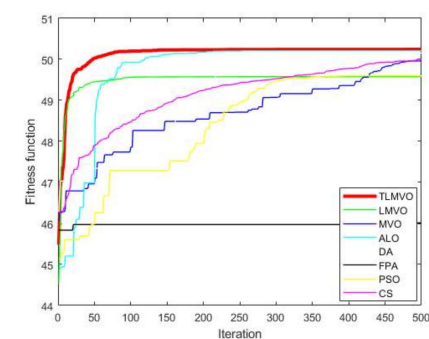

(c1) The values of Masi entropy under eight algorithms in Image 3

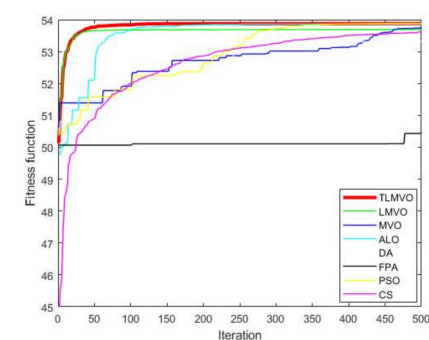

(e1) The values of Masi entropy under eight algorithms in Image 5

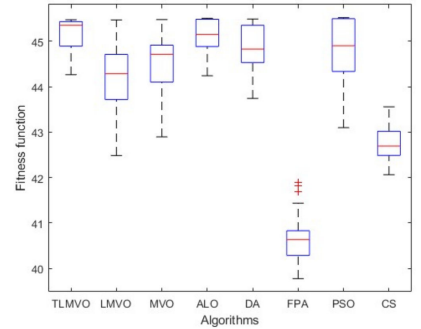

(a2) Box chart of fitness function value under eight algorithms in Image 1

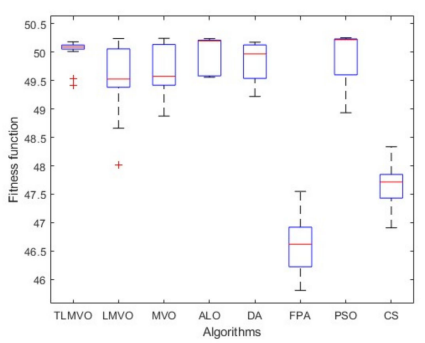

(c2) Box chart of fitness function value under eight algorithms in Image 3

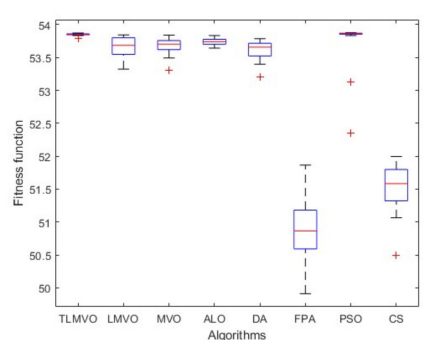

(e2) Box chart of fitness function values under eight algorithms in Image 5

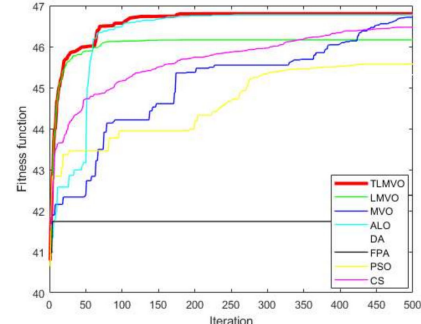

(b1) The values of Masi entropy under eight algorithms in Image 2

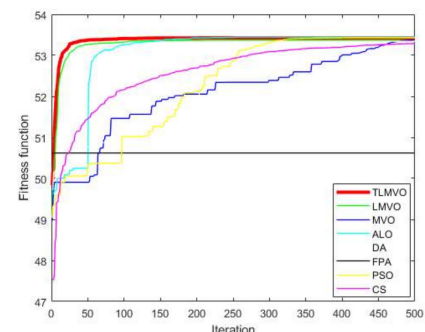

(d1) The values of Masi entropy unde eight algorithms in Image 4

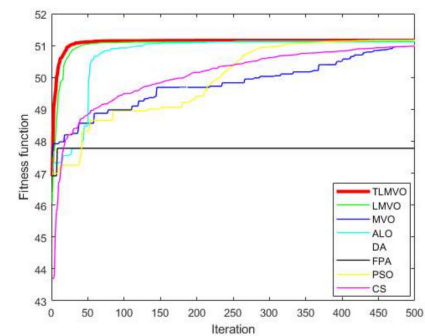

(f1) The values of Masi entropy under eight algorithms in Image 6

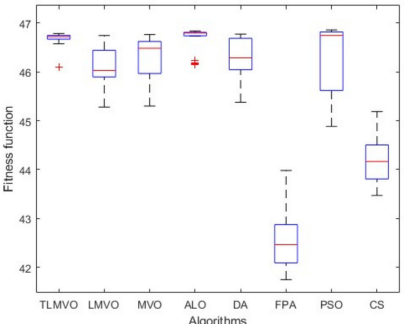

(b2) Box chart of fitness function value under eight algorithms in Image 2

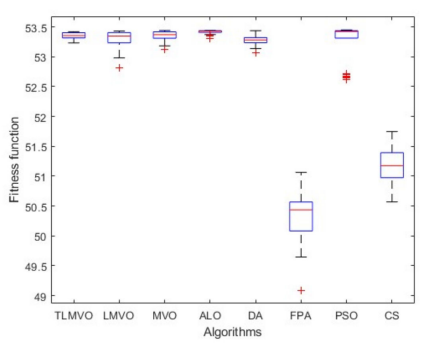

(d2) Box chart of fitness function value under eight algorithms in Image 4

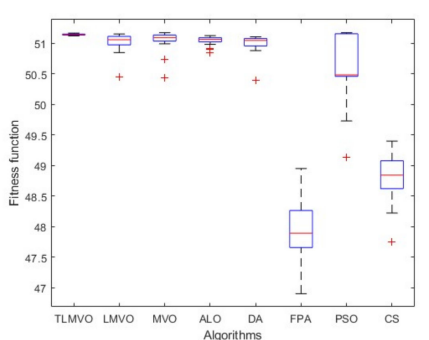

(f2) Box chart of fitness function values under eight algorithms in Image 6

Figure 10. Cont. 


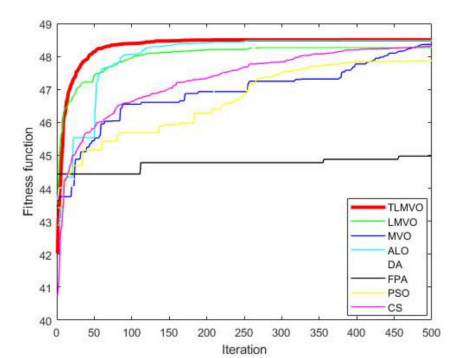

(g1) The values of Masi entropy under eight algorithms in Image 7

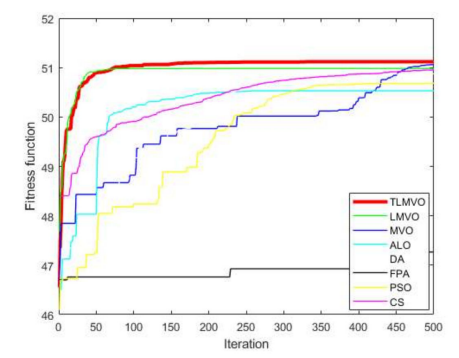

(i1) The values of Masi entropy under eight algorithms in Image 9

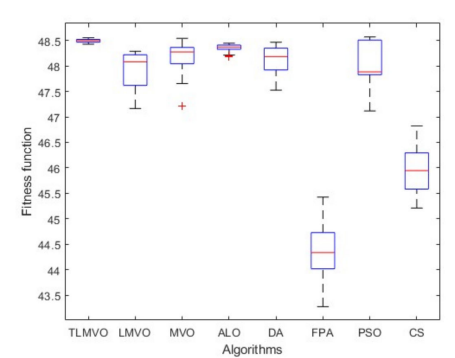

(g2) Box chart of fitness function values under eight algorithms in Image 7

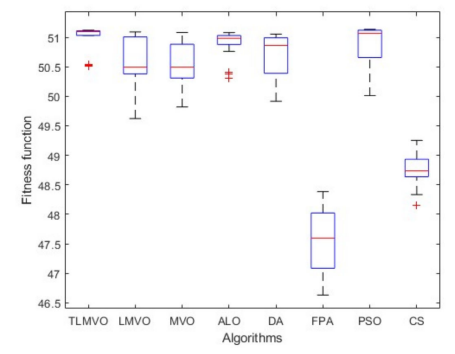

(i2) Box chart of fitness function value under eight algorithms in Image 9

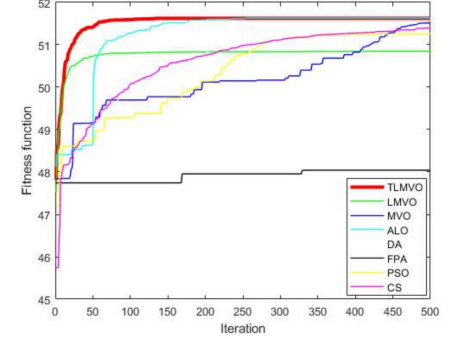

(h1) The values of Masi entropy unde eight algorithms in Image 8

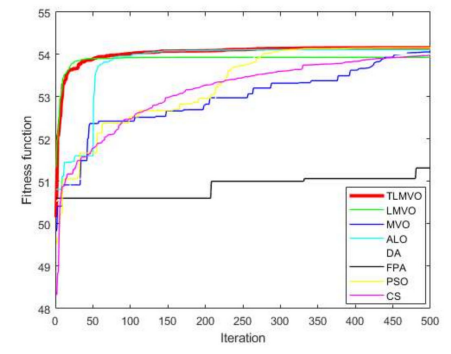

(j1) The values of Masi entropy under eight algorithms in Image 10

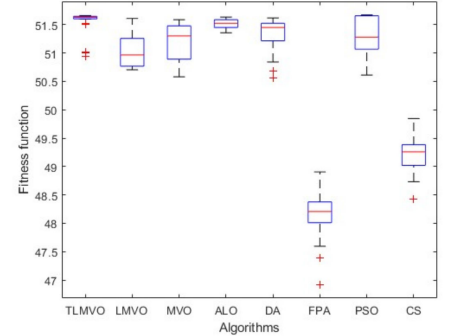

(h2) Box chart of fitness function values under eight algorithms in Image 6

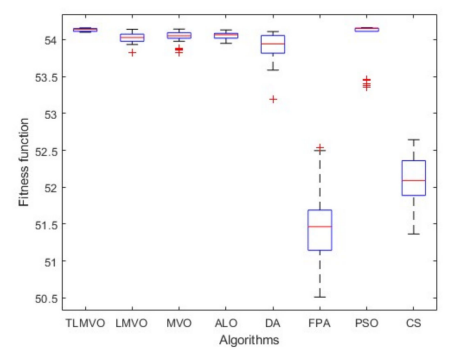

(j2) Box chart of fitness function value under eight algorithms in Image 10

Figure 10. The fitness function curves and box charts obtained by the TLMVO method of 10 satellite images 
As the fitness function of the application, a Masi entropy mathematical model is non-extensive and additive, which can provide better threshold results than other segmentation methods [23]. In the table of fitness function values, Table 8, it can be seen that PSO as a basic optimization algorithm can obtain better results at low thresholds. At high thresholds, the results of TLMVO, LMVO, MVO, ALO and DA have little difference. According to the label distribution, LMVO and ALO can be regarded as the second best. Figure 10 presents that:

1. In terms of convergence curve: in the early stage, TLMVO did not rapidly obtain a large value in the first 100 generations, as shown in Figure 10a1,b1,e1,i1,j1. In the 200th generation, TLMVO algorithm is faster than other algorithms to obtain the maximum target value or close to the theoretical maximum target value, as shown in Figure 10a1,b1,e1,g1,i1,j1. After the 250th generation, other algorithms are largely not updated.

However, for TLMVO, the advantages of ALO and CS position updating are used for reference, and mutation factors are added to maintain good population diversity and continuous updating in the later period of operation. As an improvement of the LMVO algorithm, TLMVO still retains the advantages of Lévy flight to avoid the algorithm falling into local optimization, and to be able to jump to a mutation space for optimization occasionally. On the basis of LMVO, the screening mechanism of the optimal solution is improved, and the mutation factor is added in the location update, which achieves better convergence and robustness. Traditional MVO population regeneration is slow and variation occurs at intervals. The convergence curve is stepped rather than a rising smooth curve. In addition to hybrid algorithms, ALO algorithm is always superior to other algorithms. The overall fluctuation of FPA algorithm is relatively large, while CS is relatively small. The optimal values found by them have large deviations, and some of them belong to local optimum values. Overall, TLMVO provides a competitive solution compared with other metaheuristics optimizers.

2. In terms of algorithm stability: In all box graphs, the TLMVO algorithm shows good stability, generally the best value and the second best value. For instance, the box plots in (b2) and (j2) are the second best values, and the rest are the best values, visually representing the stability of TLMVO. Other algorithms either float too much or have a lot of outliers.

In conclusion, compared with the comparison algorithm, TLMVO has higher optimization accuracy, better robustness and stability. The validity and superiority of the algorithm are proved, and the purpose of improving the basic LMVO and MVO algorithms is achieved.

\subsubsection{Complexity Analysis}

Algorithm complexity is another important indicator of performance. Complexity is related to population size, number of iterations, number of cycles, threshold size and other factors. The time complexity of TLMVO, LMVO and MVO can be expressed as $O(I * N * D) * O(F(x))$, where $I$ represents the maximum number of iterations, $N$ denotes the population size, $D$ indicates the threshold value, and $F(x)$ corresponds to the Masi entropy function in this paper. As the number of thresholds increases, the complexity of the algorithm increases and the computing time becomes longer. In order to more intuitively analyze the computational complexity and time complexity of the algorithm, CPU time (in seconds) is selected for measurement. Each algorithm runs independently 30 times, and the average running time of the experiment is recorded in Table 9. The data in the table are integrated into a broken line graph, as shown in Figure 11. The image is used to sort the running time of each algorithm visually. The temporal ordering of algorithm can be expressed as (from large to small): $A L O>C S>D A>T L M V O>M V O>L M V O>P S O>F P A$. The results are in good agreement with the conclusions because the re-selected screening mechanism converges slowly. TLMVO is more effective for image segmentation when the time of TLMVO is similar to that of other algorithms. 
Table 9. The the average CPU time of each algorithm under Masi.

\begin{tabular}{|c|c|c|c|c|c|c|c|c|c|}
\hline $\begin{array}{l}\text { TEST } \\
\text { IMAGES }\end{array}$ & $\mathbf{K}$ & TLMVO & LMVO & MVO & ALO & DA & FPA & PSO & CS \\
\hline \multirow{5}{*}{1} & 4 & 0.4930 & 0.4260 & 0.4410 & 1.7600 & 0.7950 & 0.3790 & 0.4100 & 1.3240 \\
\hline & 6 & 0.5100 & 0.4370 & 0.4640 & 2.3900 & 0.8090 & 0.3800 & 0.4120 & 1.3380 \\
\hline & 8 & 0.5430 & 0.4640 & 0.4870 & 3.0440 & 0.8180 & 0.4110 & 0.4330 & 1.3460 \\
\hline & 10 & 0.5700 & 0.4950 & 0.4990 & 3.7850 & 0.8200 & 0.4230 & 0.4520 & 1.3700 \\
\hline & 12 & 0.6170 & 0.5230 & 0.5800 & 4.3320 & 0.8430 & 0.4520 & 0.4810 & 1.3860 \\
\hline \multirow{5}{*}{2} & 4 & 0.6440 & 0.5330 & 0.6070 & 2.1550 & 1.0210 & 0.4990 & 0.5230 & 1.3950 \\
\hline & 6 & 0.6650 & 0.5820 & 0.6130 & 2.9660 & 1.0430 & 0.5130 & 0.5520 & 1.4220 \\
\hline & 8 & 0.7070 & 0.6030 & 0.6410 & 3.7150 & 1.0780 & 0.5460 & 0.5670 & 1.4390 \\
\hline & 10 & 0.7550 & 0.6430 & 0.6840 & 4.6660 & 1.1390 & 0.5700 & 0.6070 & 1.4530 \\
\hline & 12 & 0.7930 & 0.6770 & 0.7170 & 5.2850 & 1.1520 & 0.5980 & 0.6380 & 1.4920 \\
\hline \multirow{5}{*}{3} & 4 & 0.6220 & 0.5820 & 0.6100 & 2.1440 & 0.9300 & 0.5020 & 0.5550 & 1.4400 \\
\hline & 6 & 0.6970 & 0.6020 & 0.6360 & 2.9680 & 1.0870 & 0.5240 & 0.5690 & 1.4500 \\
\hline & 8 & 0.7200 & 0.6360 & 0.6660 & 3.7530 & 1.0840 & 0.5700 & 0.5950 & 1.4610 \\
\hline & 10 & 0.7650 & 0.6770 & 0.6970 & 4.6600 & 1.1190 & 0.5840 & 0.6350 & 1.4920 \\
\hline & 12 & 0.7870 & 0.7300 & 0.7480 & 5.2680 & 1.1560 & 0.6050 & 0.6550 & 1.5000 \\
\hline \multirow{5}{*}{4} & 4 & 0.6400 & 0.5820 & 0.6100 & 2.2110 & 1.0400 & 0.5080 & 0.5430 & 1.4210 \\
\hline & 6 & 0.6910 & 0.6060 & 0.6440 & 3.0820 & 1.0830 & 0.5320 & 0.5710 & 1.4480 \\
\hline & 8 & 0.7260 & 0.6310 & 0.6700 & 3.8380 & 1.1070 & 0.5680 & 0.6060 & 1.4640 \\
\hline & 10 & 0.7650 & 0.6600 & 0.6930 & 4.7410 & 1.1380 & 0.5880 & 0.6250 & 1.4880 \\
\hline & 12 & 0.8100 & 0.7010 & 0.7470 & 5.3380 & 1.1950 & 0.6230 & 0.6590 & 1.5140 \\
\hline \multirow{5}{*}{5} & 4 & 0.6370 & 0.5680 & 0.6290 & 2.6220 & 1.0290 & 0.5450 & 0.5570 & 1.4130 \\
\hline & 6 & 0.6930 & 0.5990 & 0.6450 & 2.9890 & 1.0950 & 0.5550 & 0.5700 & 1.4470 \\
\hline & 8 & 0.7370 & 0.6260 & 0.6670 & 3.7710 & 1.1270 & 0.5660 & 0.5990 & 1.4500 \\
\hline & 10 & 0.8100 & 0.6750 & 0.7180 & 4.7050 & 1.1650 & 0.5870 & 0.6270 & 1.4850 \\
\hline & 12 & 0.8180 & 0.6900 & 0.7430 & 5.3290 & 1.1440 & 0.6080 & 0.6500 & 1.5030 \\
\hline \multirow{5}{*}{6} & 4 & 0.6330 & 0.5740 & 0.5950 & 2.1830 & 0.9890 & 0.5340 & 0.5480 & 1.3790 \\
\hline & 6 & 0.6900 & 0.5910 & 0.6450 & 2.9780 & 1.0840 & 0.5450 & 0.5590 & 1.4270 \\
\hline & 8 & 0.7420 & 0.6510 & 0.6800 & 3.7890 & 1.1270 & 0.5660 & 0.5970 & 1.4570 \\
\hline & 10 & 0.7470 & 0.6610 & 0.6960 & 4.7190 & 1.1920 & 0.6110 & 0.6150 & 1.4770 \\
\hline & 12 & 0.8120 & 0.6880 & 0.7300 & 5.3230 & 1.2360 & 0.6040 & 0.6470 & 1.4960 \\
\hline \multirow{5}{*}{7} & 4 & 0.6470 & 0.5808 & 0.6000 & 2.1550 & 1.0180 & 0.5030 & 0.5440 & 1.4140 \\
\hline & 6 & 0.6670 & 0.5900 & 0.6290 & 3.0620 & 1.1050 & 0.5510 & 0.5840 & 1.4260 \\
\hline & 8 & 0.7170 & 0.6530 & 0.6900 & 5.1410 & 1.2440 & 0.6050 & 0.6350 & 1.4920 \\
\hline & 10 & 0.8270 & 0.7050 & 0.7550 & 5.4410 & 1.4680 & 0.6610 & 0.6770 & 1.6110 \\
\hline & 12 & 0.9650 & 0.7880 & 0.7980 & 6.1020 & 1.3370 & 0.6390 & 0.6560 & 1.5610 \\
\hline \multirow{5}{*}{8} & 4 & 0.7020 & 0.6280 & 0.6550 & 2.5230 & 1.1429 & 0.4980 & 0.5840 & 1.4040 \\
\hline & 6 & 0.7360 & 0.6420 & 0.6730 & 3.5880 & 1.1470 & 0.5220 & 0.5910 & 1.4260 \\
\hline & 8 & 0.7740 & 0.6540 & 0.6970 & 4.0550 & 1.1490 & 0.5480 & 0.6030 & 1.4440 \\
\hline & 10 & 0.7890 & 0.6740 & 0.7460 & 5.2380 & 1.1510 & 0.5840 & 0.6230 & 1.4690 \\
\hline & 12 & 0.8440 & 0.7200 & 0.7390 & 5.8670 & 1.2320 & 0.6270 & 0.6910 & 1.5100 \\
\hline \multirow{5}{*}{9} & 4 & 0.7210 & 0.6390 & 0.6920 & 2.4750 & 1.0250 & 0.5920 & 0.6150 & 1.4800 \\
\hline & 6 & 0.7860 & 0.6680 & 0.7560 & 3.3060 & 1.1820 & 0.5950 & 0.6330 & 1.5070 \\
\hline & 8 & 0.8630 & 0.7210 & 0.7930 & 4.3700 & 1.3930 & 0.6080 & 0.6470 & 1.5110 \\
\hline & 10 & 0.8900 & 0.7300 & 0.7660 & 5.5070 & 1.2570 & 0.6690 & 0.7070 & 1.5300 \\
\hline & 12 & 0.9590 & 0.7690 & 0.8210 & 6.3310 & 1.4100 & 0.6870 & 0.7280 & 1.6470 \\
\hline \multirow{5}{*}{10} & 4 & 0.6838 & 0.5580 & 0.6020 & 2.2140 & 1.0170 & 0.5180 & 0.5410 & 1.4290 \\
\hline & 6 & 0.7070 & 0.5880 & 0.6400 & 3.0660 & 1.0700 & 0.5400 & 0.5790 & 1.4500 \\
\hline & 8 & 0.7390 & 0.6250 & 0.6490 & 3.8670 & 1.1080 & 0.5900 & 0.6110 & 1.4710 \\
\hline & 10 & 0.7790 & 0.6790 & 0.7050 & 4.8330 & 1.1280 & 0.6100 & 0.6390 & 1.4940 \\
\hline & 12 & 0.8320 & 0.7110 & 0.7590 & 5.5270 & 1.1810 & 0.6320 & 0.6690 & 1.5140 \\
\hline
\end{tabular}




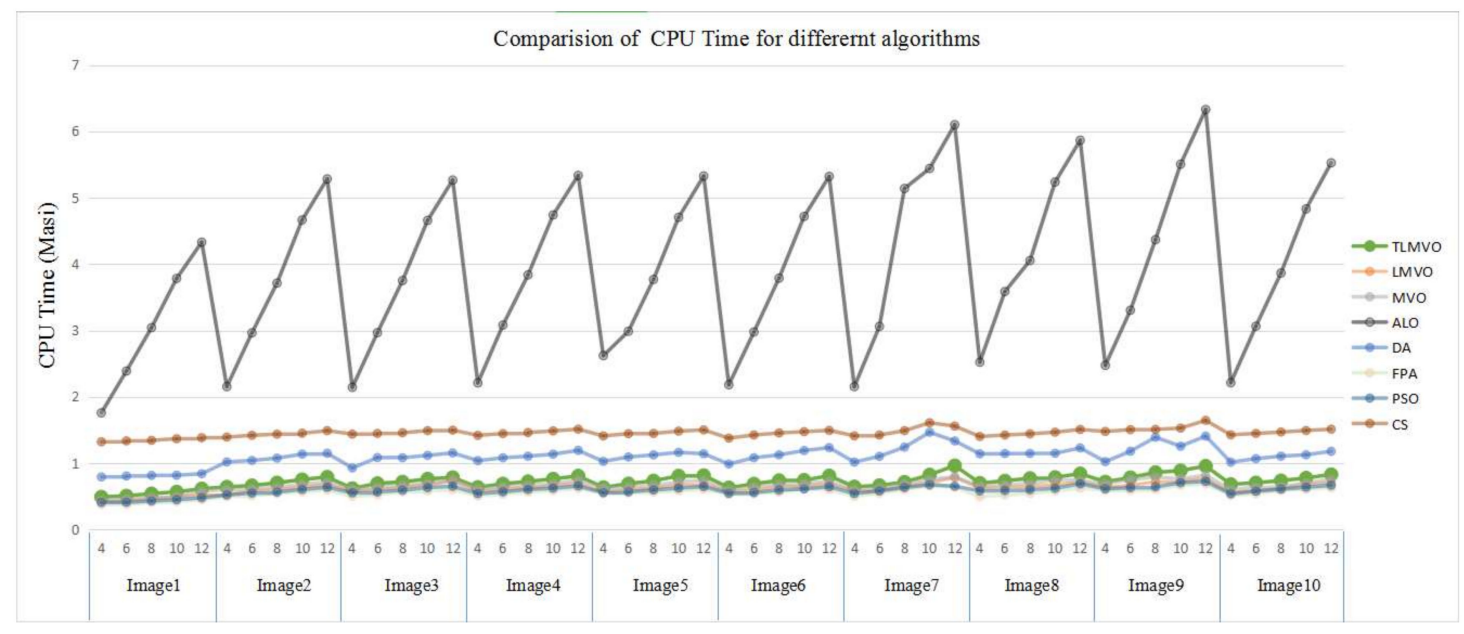

Figure 11. Broken line chart of the CPU Time indicator.

\subsubsection{Statistical Analysis}

In order to better analyze the results, we chose two more secure data statistical tests, namely Wilcoxon's rank sum test and Friedman test.

1. Wilcoxon's rank sum test is a pair-wise test, which aims to detect the significant difference between the mean values of two samples. In this paper, they correspond to the behavior of the two algorithms. The fitness function of GSMVO $(\mathrm{K}=12)$ algorithm is compared with other seven algorithms. All algorithms run the same 30 times. The corresponding results are given in Table 10. The probability of a statistical value, the $p$-value and the indicator $h$ are set throughout the test to determine whether to accept or reject the null hypothesis. Let the null hypothesis be: "There is no significant difference between the proposed algorithm and other algorithms." If the $p$-value is $>0.05 \mathrm{or} h=0$, accept the null hypothesis, otherwise reject it. In addition, 67 of the 70 cases achieved superior results, which indicates that there is a significant difference between TLMVO and the other seven algorithms. In most cases, the TLMVO-based multilevel threshold algorithm outperforms the other seven algorithms.

Table 10. Average $p$-value of Wilcoxon test after 30 times of operation under Masi. (The data of $p>0.05$ has been bolded, "+" indicates significant difference.).

\begin{tabular}{|c|c|c|c|c|c|c|c|c|c|c|c|c|c|c|}
\hline \multirow{2}{*}{$\begin{array}{c}\text { TEST } \\
\text { IMAGES }\end{array}$} & \multicolumn{2}{|c|}{$\begin{array}{c}\text { TLMVO } \\
\text { vs. LMVO }\end{array}$} & \multicolumn{2}{|c|}{$\begin{array}{l}\text { TLMVO } \\
\text { vs. MVO }\end{array}$} & \multicolumn{2}{|c|}{$\begin{array}{l}\text { TLMVO } \\
\text { vs. ALO }\end{array}$} & \multicolumn{2}{|c|}{$\begin{array}{l}\text { TLMVO } \\
\text { vs. DA }\end{array}$} & \multicolumn{2}{|c|}{$\begin{array}{l}\text { TLMVO } \\
\text { vs. FPA }\end{array}$} & \multicolumn{2}{|c|}{$\begin{array}{l}\text { TLMVO } \\
\text { vs. PSO }\end{array}$} & \multicolumn{2}{|c|}{$\begin{array}{c}\text { TLMVO } \\
\text { vs. CS }\end{array}$} \\
\hline & $p$ & h & $p$ & h & $p$ & h & $p$ & h & $p$ & h & $p$ & h & $p$ & h \\
\hline 1 & $<0.05+$ & 1 & $<0.05$ & 1 & $<0.05+$ & 1 & $<0.05+$ & 1 & $<0.05+$ & 1 & $<0.05+$ & 1 & $<0.05+$ & 1 \\
\hline 2 & $<0.05+$ & 1 & $<0.05+$ & 1 & 0.057 & 0 & $<0.05+$ & 1 & $<0.05+$ & 1 & $<0.05+$ & 1 & $<0.05+$ & 1 \\
\hline 3 & $<0.05$ & 1 & $<0.05$ & 1 & $<0.05$ & 1 & $<0.05+$ & 1 & $<0.05+$ & 1 & $<0.05$ & 1 & $<0.05+$ & 1 \\
\hline 4 & $<0.05+$ & 1 & $<0.05$ & 1 & $<0.05+$ & 1 & $<0.05+$ & 1 & $<0.05+$ & 1 & $<0.05+$ & 1 & $<0.05+$ & 1 \\
\hline 5 & $<0.05+$ & 1 & $<0.05$ & 1 & $<0.05+$ & 1 & $<0.05+$ & 1 & $<0.05+$ & 1 & $<0.05+$ & 1 & $<0.05+$ & 1 \\
\hline 6 & $<0.05+$ & 1 & $<0.05$ & 1 & $<0.05$ & 1 & $<0.05+$ & 1 & $<0.05+$ & 1 & $<0.05+$ & 1 & $<0.05+$ & 1 \\
\hline 7 & 0.1952 & 0 & $<0.05$ & 1 & 0.307 & 0 & $<0.05+$ & 1 & $<0.05+$ & 1 & 0.0072 & 1 & $<0.05+$ & 1 \\
\hline 8 & $<0.05+$ & 1 & $<0.05$ & 1 & $<0.05+$ & 1 & $<0.05+$ & 1 & $<0.05+$ & 1 & $<0.05+$ & 1 & $<0.05+$ & 1 \\
\hline 9 & $<0.05+$ & 1 & $<0.05$ & 1 & $<0.05$ & 1 & $<0.05+$ & 1 & $<0.05+$ & 1 & $<0.05+$ & 1 & $<0.05+$ & 1 \\
\hline 10 & $<0.05+$ & 1 & $<0.05$ & 1 & $<0.05$ & 1 & $<0.05+$ & 1 & $<0.05+$ & 1 & $<0.05$ & 1 & $<0.05+$ & 1 \\
\hline
\end{tabular}

2. Friedman test can be used to test the overall performance of data. The null hypothesis test approximate parameters are: $H_{0}$, the median of the equality between the algorithms; $H_{1}$, which is the alternative hypothesis, used to show the degree of difference; $\alpha$ : the rejection probability of the null hypothesis when the null hypothesis is true. If the $p$-value is less than the significance level, $H_{0}$ is rejected. A detailed description is in [67]. At one time, the rank serial numbers of the whole algorithm will be tested with the corresponding index data of the algorithm. It reflects the overall performance of 
the algorithm intuitively and quickly. We put all the index data mentioned above into the test and get Table 11 (the highest ranking is marked with shadows). From the rankings obtained, TLMVO shows superiority in any threshold of different pictures, although sometimes the rankings are very close or the same.

After all experiments and results analysis, it can be concluded that TLMVO has greatly improved on LMVO and MVO. Compared with other metaheuristics algorithms, the TLMVO algorithm has better accuracy, convergence and robustness in multi-threshold color satellite image segmentation. This method can be used as an effective method for multilevel image threshold segmentation.

Table 11. The Friedman test of each algorithm under Masi.

\begin{tabular}{|c|c|c|c|c|c|c|c|c|c|}
\hline $\begin{array}{l}\text { TEST } \\
\text { IMAGES }\end{array}$ & $\mathbf{K}$ & TLMVO & LMVO & MVO & ALO & DA & FPA & PSO & CS \\
\hline \multirow{5}{*}{ 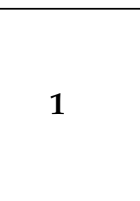 } & 4 & 1.8 & 3.2 & 4.2 & 5.3 & 3.8 & 6.4 & 4.1 & 7.2 \\
\hline & 6 & 3 & 3.4 & 3.4 & 5 & 3.4 & 6.6 & 4.4 & 6.8 \\
\hline & 8 & 2.8 & 3.6 & 4.2 & 3.2 & 5.2 & 6.6 & 3.4 & 7 \\
\hline & 10 & 2.6 & 4.4 & 4 & 3 & 4 & 6 & 4.4 & 7.6 \\
\hline & 12 & 1.8 & 2.6 & 4.6 & 3.6 & 4.8 & 6.6 & 5 & 7 \\
\hline \multirow{5}{*}{2} & 4 & 2.4 & 3.6 & 4.2 & 4.6 & 4 & 5.2 & 6 & 6 \\
\hline & 6 & 2.4 & 5.4 & 3.4 & 3.4 & 4 & 6.6 & 3.8 & 7 \\
\hline & 8 & 2.8 & 3.4 & 4.4 & 3.2 & 5.2 & 6.4 & 3.4 & 7.2 \\
\hline & 10 & 1.8 & 2.8 & 4 & 4.8 & 6 & 6.6 & 3 & 7 \\
\hline & 12 & 1.8 & 4.2 & 4.6 & 3.4 & 3.4 & 6.6 & 5 & 7 \\
\hline \multirow{5}{*}{3} & 4 & 2.6 & 4.4 & 3.8 & 5.4 & 3.2 & 6.6 & 4.2 & 5.8 \\
\hline & 6 & 1.8 & 3.7 & 3.4 & 6 & 4.7 & 6.6 & 3.4 & 6.4 \\
\hline & 8 & 2.8 & 3.4 & 4.8 & 3.6 & 4 & 6 & 3.8 & 7.6 \\
\hline & 10 & 1.8 & 3.8 & 4.1 & 3.4 & 4.4 & 6.6 & 5.1 & 6.8 \\
\hline & 12 & 1.8 & 4 & 4.4 & 4.6 & 5.4 & 6.6 & 2.2 & 7 \\
\hline \multirow{5}{*}{4} & 4 & 2 & 3.2 & 3.4 & 5.5 & 4.4 & 6.6 & 4.3 & 6.6 \\
\hline & 6 & 2.5 & 3.9 & 3.7 & 4.3 & 5.8 & 6.5 & 4 & 5.5 \\
\hline & 8 & 1.9 & 2.9 & 3 & 4.4 & 6 & 6.6 & 4.2 & 7 \\
\hline & 10 & 1 & 4.2 & 4.6 & 4 & 7 & 6.4 & 2.4 & 6.6 \\
\hline & 12 & 1.8 & 3 & 3.4 & 5.4 & 6 & 5.4 & 3.6 & 7.4 \\
\hline \multirow{5}{*}{5} & 4 & 2.8 & 4.2 & 5.9 & 4.4 & 6.4 & 4 & 3.2 & 5.1 \\
\hline & 6 & 2.4 & 3 & 5.2 & 4.2 & 3.6 & 6 & 4 & 7.6 \\
\hline & 8 & 1.5 & 3.3 & 3.4 & 5.6 & 5.6 & 6.6 & 3 & 7 \\
\hline & 10 & 1.8 & 3 & 4 & 3.4 & 5.8 & 6 & 4.4 & 7.6 \\
\hline & 12 & 2.8 & 3.8 & 4.8 & 4.2 & 3.2 & 6.6 & 3.6 & 7 \\
\hline \multirow{5}{*}{6} & 4 & 1.8 & 3.4 & 3.6 & 4.4 & 6 & 6.6 & 3.2 & 7 \\
\hline & 6 & 2.4 & 3.4 & 3.3 & 4.6 & 5.4 & 6.6 & 4.3 & 6 \\
\hline & 8 & 1.8 & 4 & 4 & 4.4 & 3.4 & 6.6 & 4.8 & 7 \\
\hline & 10 & 2.4 & 3.4 & 4.4 & 4.6 & 5.4 & 6.6 & 2.8 & 6.4 \\
\hline & 12 & 2.4 & 2.9 & 4.8 & 3 & 5.4 & 6.6 & 3.9 & 7 \\
\hline \multirow{5}{*}{7} & 4 & 2.6 & 3.8 & 4 & 3.8 & 6.2 & 5.6 & 4 & 6 \\
\hline & 6 & 1.8 & 4.4 & 5.1 & 4.5 & 4.8 & 6.6 & 3.2 & 5.6 \\
\hline & 8 & 2 & 3.8 & 5 & 3.4 & 5 & 6.6 & 3.2 & 7 \\
\hline & 10 & 2.6 & 3.6 & 4.3 & 3.5 & 4.4 & 6.6 & 4 & 7 \\
\hline & 12 & 1.8 & 3.2 & 4.4 & 3.6 & 5 & 6.6 & 4.4 & 7 \\
\hline \multirow{5}{*}{8} & 4 & 1.8 & 3.3 & 4.8 & 3.6 & 5.5 & 5.4 & 4 & 7.6 \\
\hline & 6 & 1.6 & 4.4 & 4.2 & 3 & 5.2 & 6.6 & 4 & 7 \\
\hline & 8 & 1.8 & 3.8 & 4 & 4 & 5.8 & 6.6 & 3 & 7 \\
\hline & 10 & 2.4 & 3.6 & 3.8 & 3.2 & 4.2 & 5.8 & 5.2 & 7.8 \\
\hline & 12 & 1.8 & 3.8 & 4.2 & 4.8 & 5.8 & 6.6 & 2 & 7 \\
\hline
\end{tabular}


Table 11. Cont.

\begin{tabular}{cccccccccc}
\hline $\begin{array}{c}\text { TEST } \\
\text { IMAGES }\end{array}$ & K & TLMVO & LMVO & MVO & ALO & DA & FPA & PSO & CS \\
\hline & $\mathbf{4}$ & 2.1 & 2.4 & 4.2 & 5.4 & 5 & 6.4 & 3.3 & 7.2 \\
$\mathbf{9}$ & $\mathbf{6}$ & 2.4 & 3.2 & 3 & 4.8 & 5.6 & 5.6 & 4.2 & 7.2 \\
& $\mathbf{8}$ & 2 & 2.7 & 3.5 & 4.6 & 6 & 6.6 & 3.6 & 7 \\
& $\mathbf{1 0}$ & 1.8 & 3.1 & 3.9 & 4.4 & 4.4 & 6 & 4.8 & 7.6 \\
& $\mathbf{1 2}$ & 1.8 & 3.8 & 4.2 & 5.8 & 3.6 & 6.4 & 3.2 & 7.2 \\
\hline & $\mathbf{4}$ & 3.4 & 3.6 & 4.5 & 5 & 3.8 & 5.2 & 4.1 & 6.4 \\
$\mathbf{1 0}$ & $\mathbf{6}$ & 2.2 & 5.6 & 4.2 & 3 & 4.4 & 6.2 & 3.2 & 7.2 \\
& $\mathbf{8}$ & 2.4 & 2.4 & 3 & 4.9 & 6 & 6.2 & 3.7 & 7.4 \\
& $\mathbf{1 0}$ & 1.8 & 2.8 & 3.8 & 4.4 & 5.6 & 6.6 & 4 & 7 \\
& $\mathbf{1 2}$ & 1.8 & 2.8 & 3.4 & 4.4 & 5.8 & 6.4 & 4.2 & 7.2 \\
\hline
\end{tabular}

\section{Conclusions}

This paper extensively studies the improved algorithm for color satellite image segmentation based on multilevel threshold. In order to solve the problem of a large amount of information and high precision of satellite image segmentation, a method combining the improved TLMVO algorithm with the much-anticipated Masi entropy in recent years is adopted. The results show that this method can be effectively applied to multilevel threshold segmentation of color images. Ten satellite images were used to test the multi-threshold performance of the algorithm. According to fitness function value, average CPU running time, Structural Similarity Index (SSIM), Feature Similarity Index (FSIM), and Peak Signal to Noise Ratio (PSNR), the segmentation results were evaluated. The results of Wilcoxon's rank sum test and Friedman test were analyzed. The validity and stability of the improved algorithm are verified by qualitative and quantitative methods. As an improvement of LMVO and MVO algorithms, Tournament selection mechanism that is more suitable for optimization algorithm was selected. Drawing on the merits of CS algorithm and ALO algorithm, the mutation factor is added to improve the position updating formula. Compared with other seven algorithms, TLMVO has better convergence and robustness. The multilevel threshold segmentation method based on TLMVO has broad application prospects. In future work, other new effective algorithms will be learned and improved, and a simpler and more efficient optimization method will be found. It is also applied to various computer vision problems such as satellite image enhancement, remote sensing image feature extraction and so on.

Author Contributions: X.P. contributed to the idea of this paper; X.P. and C.L. performed the experiments; X.P. wrote the paper; D.O. and H.J. contributed to the revision of this paper; Y.L. did the mapping; W.S. provided fund support.

Funding: This research was funded by the National Nature Science Foundation of China (No. 31470714).

Acknowledgments: The authors would like to thank the anonymous reviewers for their constructive comments and suggestions.

Conflicts of Interest: The authors declare no conflict of interest.

\section{References}

1. Wang, A.; Zhang, W.; Wei, X. A review on weed detection using ground-based machine vision and image processing techniques. Comput. Electron. Agric. 2019, 158, 226-240. [CrossRef]

2. Haindl, M.; Mikeš, S. A competition in unsupervised color image segmentation. Pattern Recognit. 2016, 57, 136-151. [CrossRef]

3. Ayala, H.V.H.; Santos, F.M.D.; Mariani, V.C. Image thresholding segmentation based on a novel beta differential evolution approach. Expert Syst. Appl. 2015, 42, 2136-2142. [CrossRef]

4. Bhandari, A.K.; Singh, V.K.; Kumar, A.; Singh, G.K. Cuckoo search algorithm and wind driven optimization based study of satellite image segmentation for multilevel thresholding using Kapur's entropy. Expert Syst. Appl. 2014, 41, 3538-3560. [CrossRef] 
5. Mlakar, U.; Potočnik, B.; Brest, J. A hybrid differential evolution for optimal multilevel image thresholding. Exp. Syst. Appl. 2016, 65, 221-232. [CrossRef]

6. Gao, H.; Kwong, S.; Yang, J.; Cao, J. Particle swarm optimization based on intermediate disturbance strategy algorithm and its application in multi-threshold image segmentation. Inf. Sci. 2013, 250, 82-112. [CrossRef]

7. Bhandari, A.K.; Kumar, A.; Singh, G.K. Tsallis entropy based multilevel thresholding for colored satellite image segmentation using evolutionary algorithms. Expert Syst. Appl. 2015, 42, 8707-8730. [CrossRef]

8. Ali, M.; Ahn, C.W.; Pant, M. Multilevel image thresholding by synergetic differential evolution. Appl. Soft Comput. 2014, 17, 1-11. [CrossRef]

9. Mittal, H.; Saraswat, M. An optimum multilevel image thresholding segmentation using non-local means 2D histogram and exponential Kbest gravitational search algorithm. Eng. Appl. Artif. Intell. 2018, 71, 226-235. [CrossRef]

10. Sezgin, M. Survey over image thresholding techniques and quantitative performance evaluation. J. Electron. Imaging 2004, 13, 146-168.

11. Otsu, N. A threshold selection method from gray-level histograms. IEEE Trans. Syst. Man Cybern. 1979, 9,62-66. [CrossRef]

12. Mohamed, H.; Merzban, M.E. Efficient solution of Otsu multilevel image thresholding: A comparative study. Exp. Syst. Appl. 2019, 116, 299-309.

13. Kapur, J.; Sahoo, P.K.; Wong, A.K. A new method forgray-level picture thresholding using the entropy of the histogram. CVGIP 1985, 29, 273-285.

14. Hinojosa, S.; Dhal, K.G.; Elaziz, M.A.; Oliva, D.; Cuevas, E. Entropy-based imagery segmentation for breast histology using the Stochastic Fractal Search. Neurocomputing 2018, 321, 201-215. [CrossRef]

15. Pal, S.K.; King, R.A.; Hashim, A.A. Automatic gray level thresholding through index of fuzziness and entropy. Pattern Recognit. Lett. 1983, 1, 141-146. [CrossRef]

16. Sarkar, S. A differential evolutionary multilevel segmentation of near infra-red images using Renyi's entropy. In Proceedings of the International Conference on Frontiers of Intelligent Computing: Theory and Applications (FICTA); Springer: Berlin/Heidelberg, Germany, 2013; Volume 199, pp. 699-706.

17. Shannon, C.E. A mathematical theory of communication. MD Comput. 1997, 14, 306-317. [PubMed]

18. Albuquerque, M.P.D.; Esquef, I.A.; Mello, A.R.G. Image thresholding using Tsallis entropy. Pattern Recognit. Lett. 2004, 25, 1059-1065. [CrossRef]

19. Sumathi, R.; Venkatesulu, M.; Arjunan, S.P. Extracting tumor in MR brain and breast image with Kapur's entropy based Cuckoo Search Optimization and morphological reconstruction filters. Biocybern. Biomed. Eng. 2018, 38, 918-930. [CrossRef]

20. Lopez-Garcia, P.; Onieva, E.; Osaba, E. A metaheuristics based in the hybridization of Genetic Algorithms and Cross Entropy methods for continuous optimization. Expert Syst. Appl. 2016, 55, 508-519. [CrossRef]

21. Pare, S.; Bhandari, A.K.; Kumar, A.; Singh, G.K. A new technique for multilevel color image thresholding based on modified fuzzy entropy and Lévy flight firefly algorithm. Comput. Electr. Eng. 2017, 70, 476-495. [CrossRef]

22. Naidu, M.S.R.; Rajesh Kumar, P.; Chiranjeevi, K. Shannon and Fuzzy entropy based evolutionary image thresholding for image segmentation. Alex. Eng. J. 2017, 57, 1643-1655. [CrossRef]

23. Masi, M. A step beyond Tsallis and Rényi entropies. Phys. Lett. 2005, 338, 217-224. [CrossRef]

24. Kandhway, P.; Bhandari, A.K. A Water Cycle Algorithm-Based Multilevel Thresholding System for Color Image Segmentation Using Masi Entropy. Circuits Syst. Signal Process. 2018, 1-49. [CrossRef]

25. Shubham, S.; Bhandari, A.K. A generalized Masi entropy based efficient multilevel thresholding method for color image segmentation. Multimed. Tools Appl. 2019, 1-42. [CrossRef]

26. Wolpert, D.H.; Macready, W.G. No free lunch theorems for optimization. Trans. Evol. Comput. 1997, 1, 67-82. [CrossRef]

27. Talbi, E.G. A taxonomy of hybrid metaheuristics. J. Heuristics 2002, 8, 541-564. [CrossRef]

28. Baniani, E.A.; Chalechale, A. Hybrid PSO and genetic algorithm for multilevel maximum entropy criterion threshold selection. Int. J. Hybrid Inf. Technol. 2013, 6, 131-140. [CrossRef]

29. Mafarja, M.M.; Mirjalili, S. Hybrid Whale Optimization Algorithm with simulated annealing for feature selection. Neurocomputing 2017, 260, 302-312. [CrossRef]

30. Mirjalili, S.; Mirjalili, S.M. A Hatamlou, Multiverse Optimizer: A nature-inspired algorithm for global optimization. Neural Comput. Appl. 2016, 27, 495-513. [CrossRef] 
31. Ali, E.E.; El-Hameed, M.A.; El-Fergany, A.A.; El-Arini, M.M. Parameter extraction of photovoltaic generating units using multi-verse optimizer. Sustain. Energy Technol. Assess. 2016, 17, 68-76. [CrossRef]

32. Jangir, P.; Parmar, S.A.; Trivedi, I.N.; Bhesdadiya, R.H. A novel hybrid Particle Swarm Optimizer with multi verse optimizer for global numerical optimization and Optimal Reactive Power Dispatch problem. Eng. Sci. Technol. Int. J. 2017, 20, 570-586. [CrossRef]

33. Wang, X.; Luo, D.; Zhao, X.; Sun, Z. Estimates of energy consumption in China using a self-adaptive multi-verse optimizer-based support vector machine with rolling cross-validation. Energy 2018, 152, 539-548. [CrossRef]

34. Elaziz, M.A.; Oliva, D.; Ewees, A.A.; Xiong, S. Multilevel thresholding-based grey scale image segmentation using multi-objective multi-verse optimizer. Expert Syst. Appl. 2019, 125, 112-129. [CrossRef]

35. Noraini, M.; Geraghty, J. Genetic algorithm performance with different selection strategies in solving TSP. World Congr. Eng. 2011, II, 4-9.

36. Medhane, D.V.; Sangaiah, A.K. Search space-based multi-objective optimization evolutionary algorithm. Comput. Electr. Eng. 2017, 58, 126-143.

37. Jia, H.; Peng, X.; Song, W.; Lang, C.; Xing, Z.; Sun, K. Multiverse Optimization Algorithm Based on Lévy Flight Improvement for Multithreshold Color Image Segmentation. IEEE Access 2019, 7, 32805-32844. [CrossRef]

38. Barrow, J.D.; Davies, P.C.W.; Harper, C.L. Science and Ultimate Reality: Quantum Theory, Cosmology and Complexity. Am. J. Phys. 2004, 74, 245-247.

39. Khoury, J.; Ovrut, B.A.; Seiberg, N.; Steinhardt, P.J.; Turok, A.N. From big crunch to big bang. Phys. Rev. D 2001, 65, 381-399. [CrossRef]

40. Yang, X.S.; Deb, S. Cuckoo Search via Lévy Flights. Mathematics 2010, 71, 210-214.

41. Joshi, A.S.; Kulkarni, O. Cuckoo search optimization-A review. Sci. Direct Mater. 2017, 4, 7262-7269. [CrossRef]

42. Yang, X.S. Flower pollination algorithm for global optimization. Unconv. Comput. Nat. Comput. 2012, 7445, 240-249.

43. Shen, L.; Fan, C.; Huang, X. Multilevel image thresholding using modified flower pollination algorithm. IEEE Access 2018, 6, 30508-30518. [CrossRef]

44. Xu, L.; Jia, H.; Lang, C.; Peng, X.; Sun, K. A novel method for multilevel color image segmentation based on dragonfly algorithm and differential evolution. IEEE Access 2019, 99, 2169-3536. [CrossRef]

45. Goldberg, D.E.; Deb, K. A comparative analysis of selection schemes used in genetic algorithms. Found. Genet. Algorithms 1991, 1, 69-93.

46. Handbook of Evolutionary Computation; IOP Publishing Ltd.: Bristol, UK; Oxford University Press: Oxford, UK, 1997.

47. Blickle, T.; Thiele, L. A Comparison of Selection Schemes used in Genetic Algorithms. Evol. Comput. 1996, 4, 361-394. [CrossRef]

48. Baker, J.E. Adaptive Selection Methods for Genetic Algorithms. In Proceedings of the First International Conference on Genetic Algorithms \& Their Applications, Pittsburg, CA, USA, 24-26 July 1985.

49. Li, J.; Tan, Y. Loser-Out Tournament-Based Fireworks Algorithm for Multimodal Function Optimization. IEEE Trans. Evol. Comput. 2017, 99. [CrossRef]

50. Das, S.; Mallipeddi, R.; Maity, D. Adaptive evolutionary programming with p-best mutation strategy(Article). Swarm Evol. Comput. 2013, 9, 58-68. [CrossRef]

51. Al-Betar, M.A.; Awadallah, M.A.; Khader, A.T.; Bolaji, A.L. Tournament-based harmony search algorithm for non-convex economic load dispatch problem. Appl. Soft Comput. 2016, 47, 449-459. [CrossRef]

52. Ralf, M.; Klafter, J. The random walk's guide to anomalous diffusion: A fractional dynamics approach. Phys. Rep. 2000, 339, 1-77.

53. Dinkar, S.K.; Deep, K. An efficient opposition based Lévy Flight Antlion optimizer for optimization problems. Int. J. Comput. Sci. 2018, 29, 119-141. [CrossRef]

54. Zhang, H.; Xie, J.; Hu, Q.; Shao, L.; Chen, T. A hybrid DPSO with Lévy flight for scheduling MIMO radar tasks. Appl. Soft Comput. 2018, 71, 242-254. [CrossRef]

55. Jain, M.; Singh, V.; Rani, A. A novel nature-inspired algorithm for optimization: Squirrel search algorithm. Swarm Evol. Comput. 2019, 44, 148-175. [CrossRef]

56. Mirjalili, S. The Ant Lion Optimizer. Adv. Eng. Softw. 2015, 83, 80-98. [CrossRef] 
57. Kennedy, J. Particle swarm optimization. In Proceedings of the ICNN'95 International Conference on Neural Networks, Perth, WA, Australia, 27 November-1 December 1995; Volume 4, pp. 1942-1948.

58. Akay, B. A study on particle swarm optimization and artificial bee colony algorithms for multilevel thresholding. Appl. Soft Comput. 2013, 13, 3066-3091. [CrossRef]

59. Liu, Y.; Mu, C.; Kou, W.; Liu, J. Modified particle swarm optimization-based multilevel thresholding for image segmentation. Soft Comput. 2015, 19, 1311-1327. [CrossRef]

60. Gao, H.; Pun, C.M.; Kwong, S. An efficient image segmentation method based on a hybrid particle swarm algorithm with learning strategy. Inf. Sci. 2016, 369, 500-521. [CrossRef]

61. Wang, M.; Wu, C.; Wang, L.; Xiang, D.; Huang, X. A feature selection approach for hyperspectral image based on modified ant lion optimizer. Knowl. Based Syst. 2019, 168, 39-48. [CrossRef]

62. The Aerial Data Set. Available online: https://landsat.visibleearth.nasa.gov/view.php?id=144523 (accessed on 10 March 2019).

63. Pare, S.; Kumar, A.; Bajaj, V.; Singh, G.K. An efficient method for multilevel color image thresholding using cuckoo search algorithm based on minimum cross entropy. Appl. Soft Comput. 2017, 61, 570-592. [CrossRef]

64. Wang, Z.; Bovik, A.C.; Sheikh, H.R.; Simoncelli, E.P. Image quality assessment: From error visibility to structural similarity. IEEE Trans. Image Process. 2004, 13, 600-612. [CrossRef]

65. Zhang, L.; Zhang, L.; Mou, X. FSIM: A Feature Similarity Index for Image Quality Assessment. IEEE Trans. Image Process. 2011, 20, 2378-2386. [CrossRef]

66. Roy, R.; Laha, S. Optimization of stego image retaining secret information using genetic algorithm with 8-connected PSNR. Procedia Comput. Sci. 2015, 60, 468-477. [CrossRef]

67. Pare, S.; Bhandari, A.K.; Kumar, A.; Singh, G.K. An optimal color image multilevel thresholding technique using grey-level co-occurrence matrix. Expert Syst. Appl. 2017, 87, 335-362. [CrossRef]

68. Oliva, D.; Hinojosa, S.; Cuevas, E.; Pajares, G.; Avalos, O.; Gálvez, J. Cross entropy based thresholding for magnetic resonance brain images using Crow Search Algorithm. Expert Syst. Appl. 2017, 79, 164-180. [CrossRef]

69. Ibrahim, R.A.; Elaziz, M.A.; Lu, S. Chaotic opposition-based grey-wolf optimization algorithm based on differential evolution and disruption operator for global optimization. Expert Syst. Appl. 2018, 108, 1-27. [CrossRef]

70. Derrac, J.; García, S.; Molina, D.; Herrera, F. A practical tutorial on the use of nonparametric statistical tests as a methodology for comparing evolutionary and swarm intelligence algorithms. Swarm Evol. Comput. 2011, 1, 3-18. [CrossRef] 